
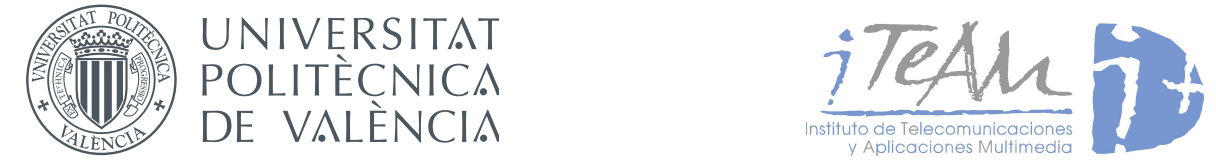

\title{
Location and Tracking for UWB In-Body Communications in Medical Applications
}

Instituto de Telecomunicaciones y Aplicaciones Multimedia Universitat Politécnica de Valéncia

A thesis for the degree of PhD in Technologies for Health and Well-Being

Valencia, November 2019

Author: Martina Barbi

Supervisors:

Prof. Narcís Cardona Marcet Dr. Concepción Garcia Pardo 

To my family, my most valuable treasure. 



\section{Abstract}

Wireless Capsule Endoscopy (WCE) is a remarkable and attractive technology adopted in the biomedical sector several years ago. It provides a non-invasive wireless imaging technology for the entire gastrointestinal (GI) tract. WCE allows specialists to recognize and diagnose diseases affecting the whole GI tract. Although physicians can receive clear pictures of abnormalities in the GI tract, they have no information about their exact location. Precise localization of the detected disorders is crucial for the subsequent removal procedure by surgery.

Currently, the frequency band allocated for capsule endoscopy applications is the MICS band $(402-405 \mathrm{MHz})$. This band offers data rate up to 500 kbps, which is insufficient to transmit high quality images. Recently, Ultrawideband (UWB) technology has been attracting attention as potential candidate for next-generation WCE systems. The advantages of UWB include simple transceiver architectures enabling low power consumption, low interference to other systems and wide bandwidth resulting in communications at higher data rate.

In this dissertation, performance of WCE localization techniques based on Radio Frequency (RF) information are investigated through software simulations, experimental laboratory measurements involving homogeneous and heterogeneous phantom models and in vivo experiments which constitute the most realistic testing scenario. Ultra-Wideband technology $(3.1-10.6 \mathrm{GHz})$ is considered as communication interface in Wireless Capsule Endoscopy. In such scenario, the wireless transmitter is located in the gastrointestinal track while one or more wireless receivers are located over the surface of the body. Received Signal Strength (RSS)-based approach is mainly explored due to its implementation simplicity and less sensitivity to bandwidth limitations. Impact of the position and the number of selected receivers on the localization accuracy is analyzed. Finally, a graphical user interface (GUI) is developed to visualize the three-dimensional (3D) localization results obtained through in vivo measurements. 



\section{Resumen}

La cápsula inalámbrica de endoscopia (WCE) es una tecnología notable y atractiva adoptada en el sector biomédico hace varios años. WCE proporciona una tecnología de imagen inalámbrica no invasiva que permite a los especialistas reconocer y diagnosticar enfermedades que afectan todo el tracto gastrointestinal. Aunque los médicos pueden recibir imágenes claras de anomalías en el tracto gastrointestinal, no tienen información sobre sus exacta ubicación. La localización precisa de los trastornos detectados es crucial para el posterior procedimiento de extracción mediante cirugía.

Actualmente, la banda de frecuencia asignada para aplicaciones de cápsula endoscópica es la banda MICS $(402-405 \mathrm{MHz})$ que ofrece una velocidad de datos de hasta $500 \mathrm{kbps}$, insuficiente para transmitir imágenes de alta calidad. Recientemente, la tecnología de banda ultra ancha (UWB) ha estado atrayendo atención como posible candidato para la próxima generación de cápsula endoscópica. Las ventajas de UWB incluyen arquitecturas de transceptor simples que permiten bajo consumo de potencia, baja interferencia a otros sistemas y amplio ancho de banda que resulta en comunicaciones a una velocidad de datos más alta.

En esta disertación, el rendimiento de las técnicas de localización de WCE basadas en radiofrecuencia (RF) se investiga a través de simulaciones software, medidas experimentales de laboratorio que involucran fantomas homogéneos y heterogéneos y a través de experimentos in vivo que constituyen el escenario de prueba más realista. La tecnología UWB (3.1-10.6 GHz) se considera como interfaz de comunicación para aplicaciones de cápsula endoscópica. En tal escenario, el transmisor inalámbrico está ubicado en el tracto gastrointestinal, mientras que uno o más receptores inalámbricos están ubicados sobre la superficie del cuerpo. El enfoque basado en la potencia recibida (RSS) se investiga principalmente debido a su simplicidad de implementación y menos sensibilidad a las limitaciones de ancho de banda. Se analiza el impacto de la posición y del número de receptores seleccionados en la precisión de la localización. Finalmente, se desarrolla una interfaz gráfica de usuario (GUI) para visualizar 
los resultados de la localización en tres dimensiones (3D) obtenidos mediante las medidas in vivo. 


\section{Resum}

La càpsula sense fil d'endoscòpia (WCE) és una tecnologia notable i atractiva adoptada en el sector biomèdic fa diversos anys. La WCE proporciona una tecnologia d'imatge sense fil no invasiva que permet als especialistes reconéixer i diagnosticar malalties que afecten tot el tracte gastrointestinal. Encara que els metges poden rebre imatges clares d'anomalies en el tracte gastrointestinal, no tenen informació sobre les seues exacta ubicació. La localització precisa dels trastorns detectats és crucial per al posterior procediment d'extracció mitjançant cirurgia.

Actualment, la banda de freqüència assignada per a aplicacions de càpsula endoscòpica és la banda MICS (402-405 MHz) que ofereix una velocitat de dades de fins a $500 \mathrm{kbps}$, insuficient per a transmetre imatges d'alta qualitat. Recentment, la tecnologia de banda ultra ampla (UWB) ha estat atraient atenció com a possible candidata per a la pròxima generació de càpsula endoscòpica. Els avantatges d' UWB inclouen arquitectures de transceptor simples que permeten un baix consum de potència, baixa interferència amb altres sistemes i una gran amplada de banda que resulta en comunicacions a una velocitat de dades més alta.

En aquesta dissertació, el rendiment de les tècniques de localització de WCE basades en radiofreqüència $(\mathrm{RF})$ s'investiga a través de simulacions amb programari, mesures experimentals de laboratori que involucren fantomes homogenis i heterogenis i a través d'experiments in vivo que constitueixen l'escenari de prova més realista. La tecnologia UWB (3.1-10.6 GHz) es considera com a interfície de comunicació per a aplicacions de càpsula endoscòpica. En tal escenari, el transmissor sense fil està situat en el tracte gastrointestinal, mentre que un o més receptors sense fils estan situats sobre la superfície del cos. L'enfocament basat en la potència rebuda (RSS) s'investiga principalment a causa de la seua simplicitat d'implementació i menys sensibilitat a les limitacions d'amplada de banda. S'analitza l'impacte de la posició i del numere de receptors seleccionats en la precisió de la localització. Finalment, es desenvolupa una interfície gràfica d'usuari (GUI) per a visualitzar els resultats de la localització en tres dimensions (3D) obtinguts mitjançant les mesures in vivo. 



\section{Acknowledgements}

First of all, I would like to thank my two advisors for their assistance and guidance. Professor Narcís Cardona received me with open arms and gave me the opportunity to be part of the 'Body Group' at the Institute of Telecommunications and Multimedia Applications (iTEAM) at the Universitat Politécnica de Valéncia (UPV). His advices have been fundamental to grow as engineer and researcher. Dr. Conchi Garcia Pardo helped and supported me throughout all these years. When I was stuck with my research she advised me with her insights and life experiences. Her guidance and encouragement gave me the strength to accomplish this work, even if sometimes it was not easy. Thanks to both of them, I could present my work to many people around the world, enjoying the experience and developing new skills.

I would like to express my deepest gratitude to my former advisors, Kamran Sayrafian and Roberto Verdone, because without them I would not have taken this path. They helped me to become a better person and a better engineer.

Besides my advisors, I would like to thank the reviewers of my thesis and the committee members for their insightful comments and suggestions.

Thank you to my colleagues of the 'Body Group', Sergio, Sofia, Alejandro and Carlos for helping me whenever I needed, for supporting me and making me grow not only as a professional, but also as a person. Thank you also to all the colleagues of the Mobile Communications Group (MCG) Gerardo, Edu, Carlos Barjau, Jordi Joan, Tere, Josetxo, Carlos Herranz, Alicia, Sonia, Irene, Jorge, Sandra and many others for making me feel part of the group.

Very special thanks go to my friends Sara, Federica, Cinzia, Lucia, Ricardo and Jessica for their support, for loving me, for believing in me and especially for standing by me during the hardest and greatest moments. I love you and I feel so lucky to have you in my life.

E senza ombra di dubbio, un ringraziamento molto speciale va alla mia famiglia, il mio piú grande sostegno e la mia guida. Senza di voi non avrei potuto realizzare i miei propositi e arrivare al punto in cui mi trovo ora. Grazie per avermi sempre sostenuta nell'affrontare ogni difficoltá, per consigliarmi nelle scelte piú difficili, per asciugarmi le lacrime durante le sconfitte, per sgri- 


\section{ACKNOWLEDGEMENTS}

darmi e per spronarmi a dare il massimo, sempre! Grazie per il vostro supporto e amore incondizionato. Vi voglio un bene immenso e cosí sará per sempre.

\section{Financial Support}

This thesis was mainly supported by the European Union's H2020:MSCA:ITN program for the "Wireless In-body Environment Communication- WiBEC" project under the grant agreement no. 675353. This thesis was also funded by the Programa de Ayudas de Investigación y Desarrollo (PAID-01-16) from Universitat Politécnica de Valéncia, by the Ministerio de Economía y Competitividad, Spain (TEC2014-60258-C2-1-R) and by the European FEDER funds and by the European Union's H2020:MSCA:ITN program for the "mmWave Communications in the Built Environments - WaveComBE" project under the grant agreement no. 766231. 


\section{Table of contents}

Abstract

$\begin{array}{ll}\text { Acknowledgements } & 11\end{array}$

$\begin{array}{ll}\text { List of Figures } & 17\end{array}$

$\begin{array}{ll}\text { List of Tables } & 21\end{array}$

1 Introduction $\quad \mathbf{2 3}$

1.1 Wireless Capsule Endoscopy . . . . . . . . . . . . . 23

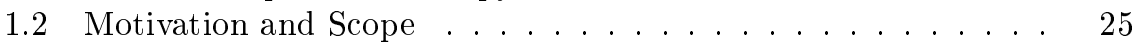

1.3 Thesis Outline . . . . . . . . . . . . . 26

1.4 Scientific Publications . . . . . . . . . . . . . 28

2 State of the Art

2.1 Overview of WCE Localization System . . . . . . . . . . . . 31

2.2 Ultra-Wideband (UWB) for next-generation WCE . . . . . . 32

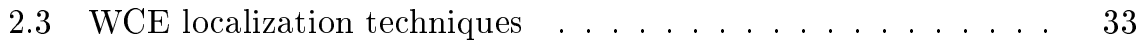

2.4 RF localization at UWB frequencies . . . . . . . . . . 37

3 Scenarios and Methodology $\quad 39$

3.1 Application Scenario . . . . . . . . . . . . . . . 39

3.2 Data Collection for RF Localization . . . . . . . . . . . . . 40

3.2.1 Frequency Domain Channel Sounding . . . . . . . . . 41

3.2.2 Experimental Laboratory Measurements . . . . . . . . 42

3.2.2.1 UWB phantom models .......... . 42

3.2.2.2 Homogeneous phantom-based campaigns . . . 44

3.2.2.3 Heterogeneous phantom-based campaign . . . 49

3.2.3 Software Simulations . . . . . . . . . . . . . . . 51

3.2.4 In Vivo Measurements . . . . . . . . . . . . . 53

3.2.4.1 Living animal subjects .......... . . . 53

3.2.4.2 First experiment . . . . . . . . . . 53 
3.2.4.3 Second experiment . . . . . . . . . . . 56

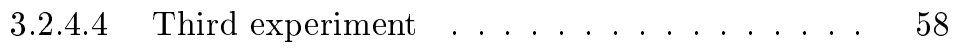

3.3 Offline RF-based Localization . . . . . . . . . . . . . . . . 61

3.3.1 Ranging distance estimation . . . . . . . . . . . . . 61

3.3.1.1 Received Signal Strength (RSS) _ . . . . . . 62

3.3.1.2 Time of Arrival $(\mathrm{ToA}) \ldots \ldots \ldots$

3.3.2 Coordinates Estimation . . . . . . . . . . . . . 64

3.3.2.1 1D Localization . . . . . . . . . . . . . 64

3.3.2.2 2D Localization . . . . . . . . . . 65

3.3.2.3 3D Localization . . . . . . . . . . . . . 66

4 Localization Results and Discussion $\quad \mathbf{7 1}$

4.1 Performance Metrics . . . . . . . . . . . . . . . 71

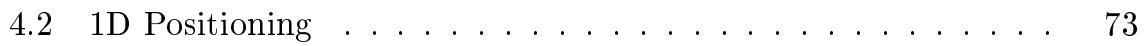

4.2 .1 RSS-based localization . . . . . . . . . . . . 73

4.2 .2 ToA-based localization . . . . . . . . . . . . . 76

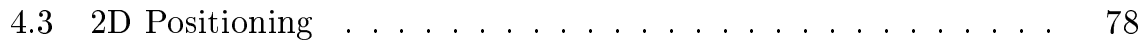

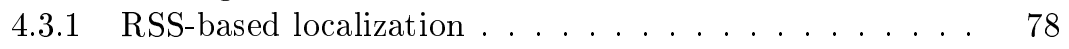

4.3.1.1 Homogeneous Phantom . . . . . . . . . . . 78

4.3.1.2 Heterogeneous Phantom . . . . . . . . . . . 80

4.3.2 ToA-based localization . . . . . . . . . . . . . . 83

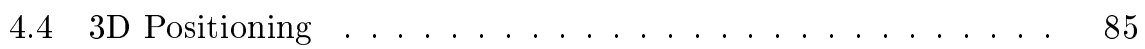

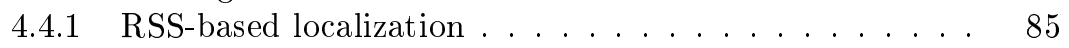

4.4.1.1 Simulations . . . . . . . . . . . . . . 85

4.4.1.2 In Vivo . . . . . . . . . . . . . . . . 89

4.4.2 Hybrid RSS/AoA localization . . . . . . . . . . 95

4.5 Effect of Receivers Distribution on Localization Accuracy . . . 98

4.6 Summary and Discussion . . . . . . . . . . . . . . . . 104

5 Real-Case Application: HemoPill Localization 107

5.1 Detection of Upper Gastrointestinal bleeding: HemoPill . . . . 107

5.1 .1 Introduction . . . . . . . . . . . . . . 107

5.1.2 Design and Working Principle . . . . . . . . . . . . 109

5.2 Measurements Setup . . . . . . . . . . . . . . . . 110

5.2 .1 Ovesco RAMCE System . . . . . . . . . . . . . . . 110

$5.2 .2 \quad$ HemoPill Testbed . . . . . . . . . . . . . . . . . . 111

5.3 RSS-based Localization Results . . . . . . . . . . . . . . . 113

6 Conclusions and Future Trends 117

6.1 Conclusions . . . . . . . . . . . . . . . . . . . 118

6.2 Future Directions . . . . . . . . . . . . . . . . . . 121

A Graphical User Interface (GUI) 123 
List of Abbreviations

References

135 



\section{List of Figures}

1.1 Example of WCE procedure [1] . . . . . . . . . . . . . 24

1.2 Example of detected pathologies through WCE procedure: (a) Crohn's disease, (b) vascular lesion, (c) polyp . . . . . . . . .

2.1 Sensors array (a) and movement pattern drawn by the software provided by Medtronic (b) [1] . . . . . . . . . . . . . 32

2.2 Localization using trilateration (a) and using triangulation (b) 34

3.1 Sensors array provided by Medtronic $[1] \ldots \ldots$. . . . . . 39

3.2 Experimental channel sounding of the IB2OB channel by using a VNA [2] . . . . . . . . . . . . . . . . . . . 41

3.3 Permittivity and conductivity of muscle phantom (a) and fat phantom (b) . . . . . . . . . . . .

3.4 Permittivity (a) and conductivity (b) of muscle phantom made of sugar and water . . . . . . . . . . . . 43

3.5 On-body (a) and in-body (b) antenna used in the measurements 44

3.6 Radiation pattern in XZ-plane (a) and simulated vs measured $S_{11}$ of in-body (b) antenna . . . . . . . . . . . 45

3.7 Radiation pattern in XZ-plane (a) and simulated vs measured $S_{11}$ of on-body (b) antenna . . . . . . . . . . 45

3.8 In-body to on-body measured grid (a) and XYZ cartesian posi-

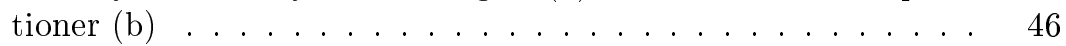

3.9 Measurement setup and in-body to on-body measured grid points 47

3.10 Magnetic tracker system (a): Electronic Unit (1), magnetic transmitter (2), magnetic sensors (3) and magnetic transmitter reference system (b) . . . . . . . . . . . . . . . .

3.11 On-body Vivaldi antenna [3] (a) and its related radiation pattern

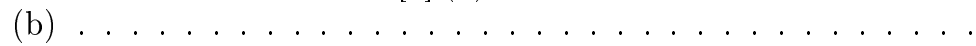

3.12 Measured reflection coefficient for the on-body Patch and Vivaldi antenna and for the in-body antenna . . . . . . . . . 48

3.13 Multilayer phantom-based setup . . . . . . . . . . . . 49 
3.14 Measured reflection coefficient, for the in-body and on-body an-

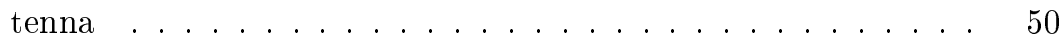

3.15 Measured grid points . . . . . . . . . . . . . . 51

3.16 On-body (a) and in-body (b) antenna locations (top view) . . . 52

3.17 Supplementary simulations: On-body (a) and in-body (b) antenna locations (top view) . . . . . . . . . . 52

3.18 First (a), and second (b) measured in-body position . . . . . . 54

3.19 In vivo measurements for one on-body position (a) and measured grid for each in-body position (top view) (b) . . . . . . . . 54

$3.20 S_{11}$ for the first (a), and second (b) measured in-body position $\quad 55$

$3.21 S_{22}$ for the first (a), and second (b) measured in-body position $\quad 55$

3.22 On-body antennas: First Patch antenna (a), Second Patch antenna (b), Directive antenna (c) . . . . . . . . . 56

3.23 Measured on-body positions (second in vivo experiment) . . . . 57

$3.24 S_{11}$ for the first (a), second (c) and third (c) measured in-body

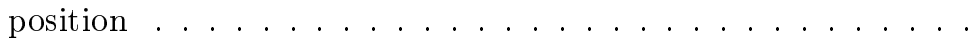

$3.25 S_{22}$ for the Patch 1 (a), Patch 2 (b) and Directive (c) antenna for the third measured in-body position . . . . . . . . .

3.26 Coplanar Waveguide (CPW) monopole (a) and tear-shape UWB

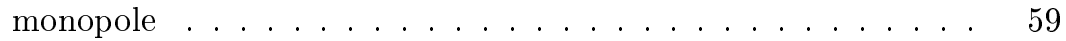

3.27 Measured on-body positions (third in vivo experiment) . . . . . 59

$3.28 S_{11}$ for the first (a) and second (b) measured in-body position . 60

$3.29 S_{22}$ for the Patch 1 (a), Patch 2 (b), CPW (c) and Tear-shape (d) 61

3.30 Third in vivo measurement setup highlighting azimuth and elevation angles .................... . . . 68

4.1 Relative errors on the estimation of $x_{I B}$ with (a) general and (c) plane related PL model for $\mathrm{z}=1.15 \mathrm{~cm}$, relative errors on the estimation of $y_{I B}$ with (b) general and (d) plane related PL model for $\mathrm{z}=1.15 \mathrm{~cm}$. . . . . . . . . . . . . . . .

4.2 CDF of relative error on the estimation of the ranging distance $d$ for (a) $\mathrm{z}=1.15 \mathrm{~cm}$, (b) $\mathrm{z}=2.15 \mathrm{~cm}$, (c) $\mathrm{z}=3.15 \mathrm{~cm} \ldots$.

4.3 Relative errors on the estimation of $x_{I B}(\mathrm{a})$ and $y_{I B}(\mathrm{~b})$ for plane $\mathrm{z}=1.15 \mathrm{~cm} \ldots \ldots \ldots \ldots \ldots \ldots$

4.4 Power Delay Profile for distance among antenna centers $d=4.02$

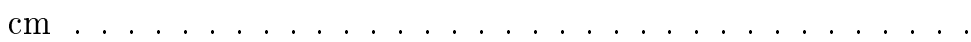

4.5 CDF of relative error on the estimation of $d$ for all $\mathrm{z}$ planes, when in-body antenna is moving along $x$-axis (a) and when is

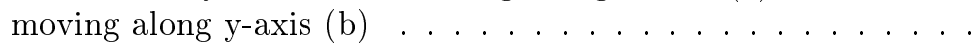

4.6 CDF of relative localization error for (a) Patch antenna using the general PL model, (b) for the Patch antenna using the related PL model, (c) for the Vivaldi antenna using the general PL model and (d) for the Vivaldi antenna using the related PL model. 
4.7 CDF of relative localization error (a), and true vs estimated location of in-body antenna (b) for 2D RSS-based localization .

4.8 CDF of relative localization error (a) and example of tracking for in-body antenna moving along $\mathrm{y}$-axis, for $\mathrm{x}=1$ and $\mathrm{z}=2$ (b), for ideal and not ideal channel estimation . . . . . . . . .

4.9 CDF of relative localization error (a) and true vs estimated location of in-body antenna (b), for 2D ToA-based localization .

4.10 Power Delay Profile for distance between antennas of $7.54 \mathrm{~cm}$.

4.11 Relative localization error vs actual in-body antenna location for different combinations of four receivers . . . . . . . . . . .

4.12 Histogram of average path loss per in-body position for different combinations of receivers . . . . . . . . . . .

4.13 Relative localization error for ideal channel estimation (a) and for not ideal channel estimation (b), considering different number of receivers .......................

4.14 Simulated path loss values and fitting model along with path loss values of selected receivers for in-body position at $5 \mathrm{~cm}$, considering ideal (a) and not ideal (b) channel estimation . . .

4.15 Measured path loss values and fitting model along with path loss values of selected receivers for in-body position IB1, considering ideal (a) and not ideal (b) channel estimation . . . . . . . . .

4.16 Measured path loss values and fitting model for Patch 1 (a), Directive (b) and Patch 2 (c) antenna . . . . . . . . .

4.17 Relative localization error obtained with the antenna related path loss model for Patch 1 (a), Directive (b) and Patch 2 (c)

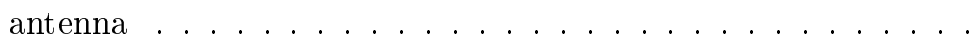

4.18 Relative localization error obtained with the general path loss model for Patch 1 (a), Directive (b) and Patch 2 (c) antenna .

4.19 Relative localization error for in-body position 1 obtained using the Patch 1 and CPW monopole antenna for RSS (a) and RSS/AoA (b) approach ...............

4.20 Relative localization error for in-body position 2 obtained using the Patch 2 and tear-shape monopole antenna for RSS (a) and RSS/AoA (b) approach ...............

4.21 Simulated path loss values and fitting model along with path loss values of selected receivers for in-body position at $6 \mathrm{~cm}$ (a) and at $7.2 \mathrm{~cm}(\mathrm{~b}) \ldots \ldots \ldots \ldots . \ldots$

4.22 In vivo measured path loss values and fitting model along with path loss values of selected receivers for in-body position 1 . . .

4.23 Laboratory measured path loss values and fitting model along with path loss values of selected receivers for in-body positions under study . . . . . . . . . . . . . . . 
4.24 Comparison between path loss values obtained through laboratory measurements, simulations and first in vivo experiment .

5.1 CAD model and photo of the bleeding sensor capsule [4] 1. recess for the entry of blood, 2. photo transistor, 3. LEDs, 4. helical antenna. Dimension of the capsule: $6.5 \mathrm{~mm}$ in diameter, 25.5 mm in length. . . . . . . . . . . . . . . 109

5.2 External system and testbed available at Ovesco [5] . . . . . . 110

5.3 HemoPill measured positions (a), measurements setup (b) and measured receiving points (c) . . . . . . . . . . . 112

5.4 Measured data along with log-distance fitting model for the $1^{\text {st }}$ (a) and $2^{\text {nd }}$ measurement campaigns ............ . .

5.5 Estimated location vs real location of HemoPill for the first measurements campaign (a) and for the second measurement cam-

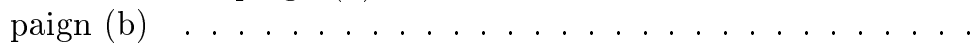

5.6 Selected path loss values for in-body position 1 for the first measurements campaign (a) and for in-body position 2 for the second

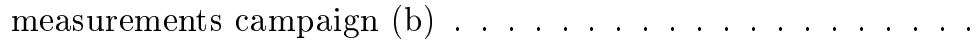

A.1 Appearance of the developed Graphical User Interface (GUI) when launched . . . . . . . . . . . . . . 125

A.2 GUI appearance when selecting First in vivo experiment . . . . 126

A.3 GUI appearance when selecting Second in vivo experiment . . 127

A.4 GUI appearance after setting all parameters . . . . . . . . . . . 128

A.5 GUI appearance after pushing Show 3D Position button for first in vivo experiment . . . . . . . . . . . . . . . . . .

A.6 GUI appearance after pushing Show 3D Position button for second in vivo experiment . . . . . . . . . . . . . . . . . . . . . . 130

A.7 Generated Localization Report after pushing Localization Error Report button ................... 131

A.8 Results opened in new figure window after pushing Open Results Figure button . . . . . . . . . . . . . . . 132 


\section{List of Tables}

4.1 Path Loss Model Parameters . . . . . . . . . . . . . . . 74

4.2 Path Loss Models for Patch and Vivaldi antenna . . . . . . . 79

4.3 Localization error for ideal and not ideal channel estimation . . 89

4.4 Localization error for different number of receivers . . . . . 90

4.5 Localization errors for in-body position IB1 . . . . . . . . . 90

4.6 Localization errors for in-body position IB2 . . . . . . . . 90

4.7 Path Loss Models for Patch 1, Patch 2, Directive antenna and for all antennas . . . . . . . . . . . . . 92

4.8 Localization errors for Patch 1, Patch 2, Directive antenna using antenna related PL models . . . . . . . . . . . 95

4.9 Localization errors for Patch 1, Patch 2, Directive antenna using the general PL model . . . . . . . . . . . . . . 95

4.10 Path Loss Models for Patch 1, CPW monopole, Patch 2 and tear-shape monopole antenna . . . . . . . . . . . . 96

4.11 Azimuth and elevation angles in degrees between in-body position 1 and Patch 1 antenna for the ten measured on-body locations 98

4.12 Localization error and average ranging error for in-body position 1 (IB1) to in-body position 4 (IB4) obtained from simulations . 99

4.13 Localization error and average ranging error for in-body position 5 (IB5) to in-body position 7 (IB7) obtained from simulations .

4.14 Localization error and average ranging error for in-body position 1 (IB1) and in-body position 2 (IB2) obtained from first in vivo experiment . . . . . . . . . . . . . . . . 101

$4.152 \mathrm{D}$ localization error and average ranging error for in-body position 1 (IB1) to in-body position 3 (IB3) obtained from heterogeneous phantom-based measurements . . . . . . . . . . 102

5.1 Path Loss Models for $1^{\text {st }}$ and $2^{\text {nd }}$ measurement campaigns . . . 113

5.2 Localization error and error on $\mathrm{x}$ estimation for the first and second measurement campaign . . . . . . . . . . . 115 



\section{Chapter 1}

\section{Introduction}

\subsection{Wireless Capsule Endoscopy}

Endoscopy is a medical procedure that allows physicians to view and examine the inside of the patient's body using an instrument called endoscope. An endoscope is a long, thin, flexible tube which has a light source and a camera at one hand. Traditional endoscopy procedures involving the Gastrointestinal (GI) tract include Upper GI endoscopy (UGD), enteroscopy and colonoscopy, depending on which portion of the digestive tract the doctor aims to inspect. UGD procedure enables the examination of the esophagus, stomach and the upper small bowel called duodenum. Enteroscopy is the endoscopic procedure for the direct visualization of the small bowel. Specifically, the first part of the small intestine, the duodenum and first portion of the jejunum, can be examined by using this longer endoscope, called enteroscope, which is introduced through the mouth and slowly advanced through the stomach, duodenum and into the jejunum by a gentle pushing action. Colonoscopy is the endoscopic examination of the large bowel and the distal part of the small bowel with a camera on a flexible tube passed through the anus. It provides a visual diagnosis (e.g. ulceration, polyps) and allows the biopsy or removal of suspected colorectal cancer lesions. Colonoscopy can remove polyps as small as one millimeter or less.

The use of capsule endoscopy is justified by the fact that all the aforementioned common practices, although very useful, are not able to access the whole small bowel [6]. Therefore, failing in providing a diagnosis, in some cases. Furthermore, traditional wired endoscopy is quite invasive, as it causes pain to the patients and it increases the risk of intestine perforation as well as of cross-contamination [7]. 
WCE has the potential to provide a non-invasive and painless way to visualize and diagnose diseases affecting the entire GI tract. This technology has been introduced by Medtronic (previously Given Imaging) in 2000 and the United States Food and Drug Administration (FDA) approved its clinical use in 2001. The WCE system is constituted by four parts, as shown in Figure 1.1, including the capsule endoscope, the data recorder, the working station, and the application software. The tiny pill has sizes between 10.8 and $11 \mathrm{~mm}$ in diameter and between 24.5 and $27.9 \mathrm{~mm}$ in length considering the commercially available capsule endoscopy devices [8]. It is equipped with a camera, an illuminating component, a battery and a wireless data transmission system that allows live transmission of images of the GI tract which can be observed remotely by physicians. As depicted in Figure 1.1, the capsule is swallowed by the patient. Then, as it travels through the digestive tract, the camera takes thousands of pictures that are transmitted to a recorder located on a belt around the patient's waist.

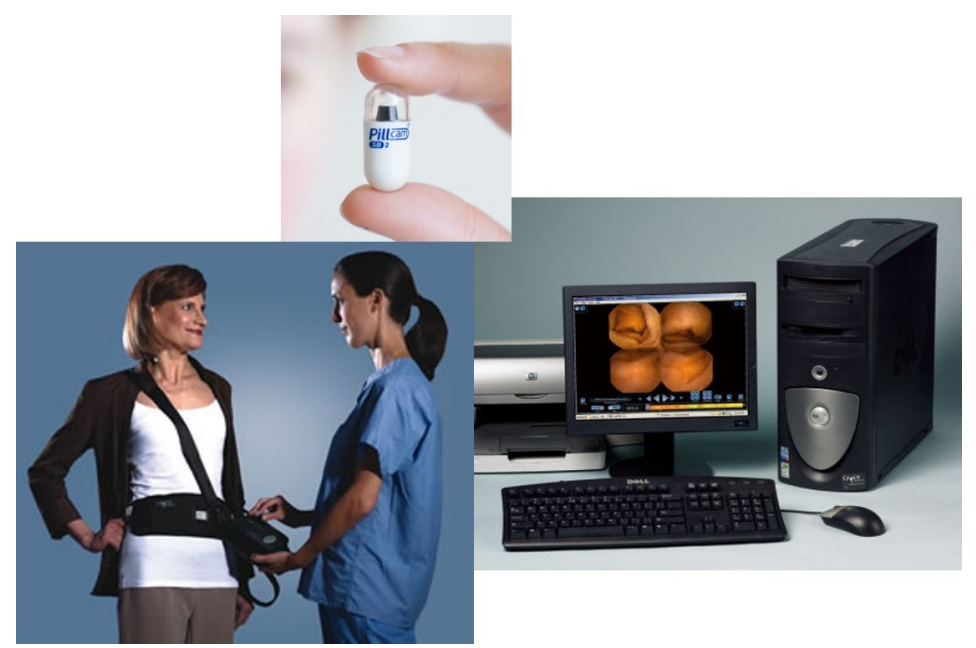

Figure 1.1: Example of WCE procedure [1]

Once the WCE's recorder is returned to the physician, the collected images are downloaded into the doctor's workstation. Then, through the software provided by the WCE company to the hospital, the doctor can visualize them as a continuous video and inspect the whole patient's small bowel. If no complications during the procedure were encountered (i.e. capsule retention), the video includes the entire patient's digestive process, from capsule ingestion to its natural expulsion. 
The specialist through the software tools can mark suspected areas as well as few reference points needed to approximately locate the pill according to its transit time. Those landmarks are the first gastric image, the first duodenum image and the passage of the capsule from the small bowel to the colon.

WCE has irreplaceable effects especially in detecting diseases along the small bowel which are the most critical to identify. Figure 1.2 shows an example of identified abnormalities through capsule endoscopy procedure.

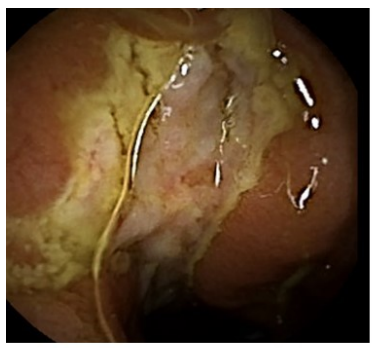

(a)

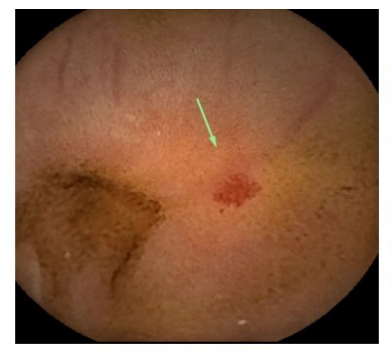

(b)

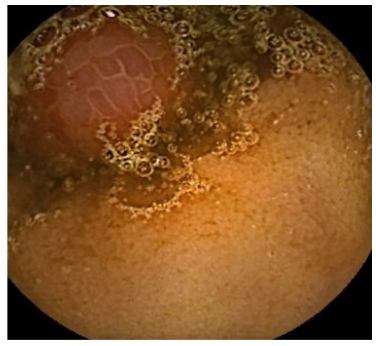

(c)

Figure 1.2: Example of detected pathologies through WCE procedure: (a) Crohn's disease, (b) vascular lesion, (c) polyp

Despite the significant advantages, WCE has also limitations. Besides possible complications during the procedure, the images sent to the recorder are not very high quality due to the currently used frequency band (Medical Implant Communication Service (MICS)) which allows low data rate. Furthermore, physicians have very little or no information about the exact location of detected diseases. Precise localization of potential abnormalities is crucial for the subsequent treatment through surgery or drug delivery.

\subsection{Motivation and Scope}

Ultra-Wideband (UWB) technology has emerged in the latest years as potential candidate for next-generation wireless capsule endoscopy applications in order to overcome the limitations of the current frequency band (Medical Implant Communication Service (MICS) band). Localization of the capsule endoscope at UWB frequencies has been limitedly explored in literature, mainly through software simulations.

For the localization process, it is desirable using the same radio frequency ( $\mathrm{RF}$ ) signal that the capsule also uses to send images to the recorder, in order to keep the hardware of the capsule simple. 
The goal of this thesis is the investigation, development and testing of location techniques based on radio frequency information for in-body communications using Ultra-Wideband technology as communication interface for Wireless Capsule Endoscopy applications. In such scenario, the wireless transmitter is located inside the gastrointestinal track while one or more wireless receivers are located over the surface of the body.

This thesis investigates the adequateness of existing radio frequency based localization approaches for indoor applications in order to adapt and optimize them for in-body communications. Such techniques involve the exploitation of channel diversity as well as some other important parameters of the propagation channel. The considered radio localization techniques are: Time of Arrival (ToA), Received Signal Strength (RSS), hybrid combination of RSS and Angle of Arrival (AoA). These localization algorithms imply a previous characterization of the propagation channel. To accomplish this, adequate UWB channel models for in-body scenarios are derived.

Selected localization techniques are developed and tested through different methodologies:

- Theory-based approach based on software simulations using digital human models.

- Experiment-based approach which involves laboratory measurements using chemical compounds (phantoms) mimicking the electromagnetic properties of the human tissues at the UPV facilities.

- In vivo measurements to validate the results obtained by theoretical and experimental approach.

Localization results obtained using the three above described approaches are analyzed and compared. Furthermore, the impact of the position and the number of selected receivers on the localization accuracy is analyzed. Finally, a graphical user interface (GUI) developed to visualize the Three-Dimensional (3D) localization results obtained through in vivo experiments is presented.

\subsection{Thesis Outline}

The organization and content of each chapter of this thesis is summarized below.

Chapter 2 presents an overview of the system used to locate the capsule endoscope in current WCE procedures. Next, UWB technology is presented, as possible candidate for next generation capsule endoscopy. Particularly, advantages and drawbacks are highlighted compared to the currently used MICS operating frequency band. Finally, a review of the existing WCE localization 
techniques is presented, by giving particular emphasis, in the last section, to RF-based approach at UWB frequencies, which is the topic of this thesis.

Chapter 3 begins by describing the measurement scenario used to emulate the real WCE procedure, for localization purposes. Next, data collection through laboratory measurements, simulations and in vivo experiments is detailed. For laboratory measurements, a novel customized testbed, including a heterogeneous phantom container used to better emulate the complex in-body environment is presented. In vivo measurements were carried out in collaboration with the Hospital La Fe, in Valencia and for the first time (to the best of the author's knowledge) used to perform RF-based localization for wireless capsule endoscopy applications. The last section of the chapter is devoted to explain the implemented approaches used for one-dimensional, two-dimensional and three-dimensional localization from the performed measurement campaigns.

Chapter 4 firstly introduces the performance metrics used to evaluate the localization accuracy. Secondly, results from simulations, laboratory and in vivo measurements obtained for one-dimensional, two-dimensional and threedimensional positioning using the Received Signal Strength (RSS), Time of Arrival (ToA) and Received Signal Strength/Angle of Arrival (RSS/AoA) approach are reported and compared. For the RSS-based method, performance are evaluated and compared under ideal and not ideal channel estimation assumption. Furthermore, the impact of receivers selection as well as of the evaluated path loss models on the localization accuracy is investigated for the three methodologies used to collect data. Finally, the last section concludes the chapter by summarizing and discussing the obtained results.

Chapter 5 describes experimental measurements carried out within a short research period at Ovesco Endoscopy company, in Tübingen, Germany. Measurements were performed using a robot assisted system available at the company with the aim of locating an ingestible wireless device specifically designed by Ovesco to detect upper gastrointestinal bleeding. The capsule was designed to operate at ISM frequencies therefore no Ultra-Wideband is considered in this case. The chapter firstly introduces the wireless device and its functionality as well as a detailed description of the measurement setup, including the liquid phantom model developed for this specific campaign. Finally, localization results obtained through RSS-based approach are presented and discussed.

Chapter 6 concludes this thesis by summarizing the general conclusions and the main findings. Future research lines derived from this work are also cited at the end of this chapter. 


\subsection{Scientific Publications}

\section{International Conferences}

- M. Barbi, S. Perez-Simbor, C. Garcia-Pardo, C. Andreu, N. Cardona, "Localization for Capsule Endoscopy at UWB Frequencies using an Experimental Multilayer Phantom", in IEEE Wireless Communications and Networking Conference (WCNC), Barcelona, 2018, pp. 390-395

- M. Barbi, C. Garcia-Pardo, N. Cardona, A. Nevarez, V. Pons, M. Frasson, "Impact of Receivers Location on the Accuracy of Capsule Endoscope Localization" in IEEE International Symposium on Personal, Indoor and Mobile Radio Communications (PIMRC), Bologna, 2018, pp. 340-344

- M.Barbi, S. Perez-Simbor, C. Garcia-Pardo, N. Cardona, "Analysis of the Localization Error for Capsule Endoscopy Applications at UWB Frequencies", in 2019 13th International Symposium on Medical Information and Communication Technology (ISMICT), Oslo, Norway, 2019, pp. 1-6

- S. Perez-Simbor, M. Barbi, C. Garcia-Pardo, S. Castelló-Palacios and N. Cardona, "Initial UWB in-body channel characterization using a novel multilayer phantom measurement setup", in IEEE Wireless Communications and Networking Conference Workshops (WCNCW), Barcelona, 2018, pp. 384-389

- S. Perez-Simbor, M. Barbi, M. Ramzan, X. Fang, C. Garcia-Pardo, N. Cardona, Q. Wang, N. Neumann, D. Plettemeier, "Experimental Path loss models comparison and localization of Wireless Endoscopic Capsule in the Ultra Wideband Frequency Band", in BODYNETS 2018 13th EAI International Conference on Body Area Networks, October 2-3, Oulu, 2018. p. accepted for publication

\section{National Conference}

- C. Garcia-Pardo, C. Andreu-Estellés, S. Perez-Simbor, S. Castelló-Palacios, Alejandro Fornes-Leal, M. Barbi, A. Vallés-Lluch, N. Cardona, "UWB Propagation for Medical In-body Devices", XXXIII Simposium Nacional de la unión cientÃfica internacional de Radio, URSI, Granada, 2018

\section{International Journals}

- M. Barbi, C. Garcia-Pardo, A. Nevarez, V. Pons and N. Cardona, "UWB RSS-based Localization for Capsule Endoscopy using a Multilayer Phantom and In Vivo Measurements," in IEEE Transactions on Antennas and Propagation, vol. 67, no. 8, pp. 5035-5043, Aug. 2019 
- C. Garcia-Pardo, C. Andreu, A. Fornes-Leal, S. Castelló-Palacios, S. Perez-Simbor, M. Barbi, A. Vallés-Lluch, N. Cardona, "Ultrawideband Technology for Medical In-Body Sensor Networks: An Overview of the Human Body as a Propagation Medium, Phantoms, and Approaches for Propagation Analysis," in IEEE Antennas and Propagation Magazine, vol. 60, no. 3, pp. 19-33, June 2018

\section{National Journals}

- C. García-Pardo, C. Andreu, A. Fornes-Leal, S. Castelló-Palacios, S. Perez-Simbor, M. Barbi, A. Vila Jiménez, M. Cabedo-Fabres, V. Pons, M. Frasson, A. Vallés-Lluch, N. Cardona, "Wireless Communications for Medical In-Body Devices: Challenges for In-body Propagation", Waves magazine 2017

\section{Scientific Talks}

- "Localization for Capsule Endoscopy at UWB Frequencies using an Experimental Multilayer Phantom", TD (17)05023, IRACON COST, 5th MC meeting and 5th technical meeting, Graz, Austria, Sept. 2017, p. published in the 5th Newsletter of the IRACON COST Action CA15104, Inclusive Radio Communication Networks for $5 G$ and beyond

- "UWB RSS-based Localization for Capsule Endoscopy using a Multilayer Phantom and In Vivo Measurements", TD(18)07024, IRACON COST Action CA15104, Inclusive Radio Communication Networks for $5 G$ and beyond, 7th MC meeting and 7th technical meeting, Cartagena, Spain, June, 2018 



\section{Chapter 2}

\section{State of the Art}

In this chapter an overview of the localization system currently used to locate the wireless capsule endoscope is given. Next, the potential of UWB technology as communication interface for future WCE applications is pointed out. Finally, a review of the existing WCE localization techniques is presented.

\subsection{Overview of WCE Localization System}

In current WCE procedures, in order to locate the capsule endoscope, a sensors array [1], as shown in Figure 2.1(a), is placed on the patient's body to receive transmission data from the pill. Particularly, the information collected by the sensors is used by the software to draw a movement pattern (Figure 2.1(b)), as the capsule travels along the GI tract. Position estimation of the pill through this system is based on off-line processing of the strength of the radio frequency signals emitted from the capsule and received by each of the eight sensors (Figure 2.1(a)) [1]. Particularly, the three sensors experiencing the highest signal strength are used for localization.

The relative two-dimensional (2D) location of the capsule, as for the Medtronic software, is estimated with respect to the umbilicus (e.g. abdominal quadrant). The localization software was studied in a series of 17 healthy subjects [9]. Multiple fluoroscopic images (92 sets) were obtained at various times during the video capsule's passage through the small bowel. The location was assessed in two dimensions relative to the umbilicus and then compared to the position obtained from the localization software. When compared to the relative $2 \mathrm{D}$ location determined fluoroscopically, approximately $87 \%(80 / 92)$ of the video capsule estimates were within $6 \mathrm{~cm}$ (a "fist"). The mean error for video capsule localization was found to be $3.8 \mathrm{~cm} \mathrm{[9]}$. 


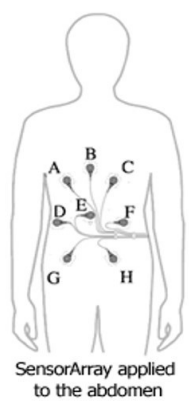

(a)

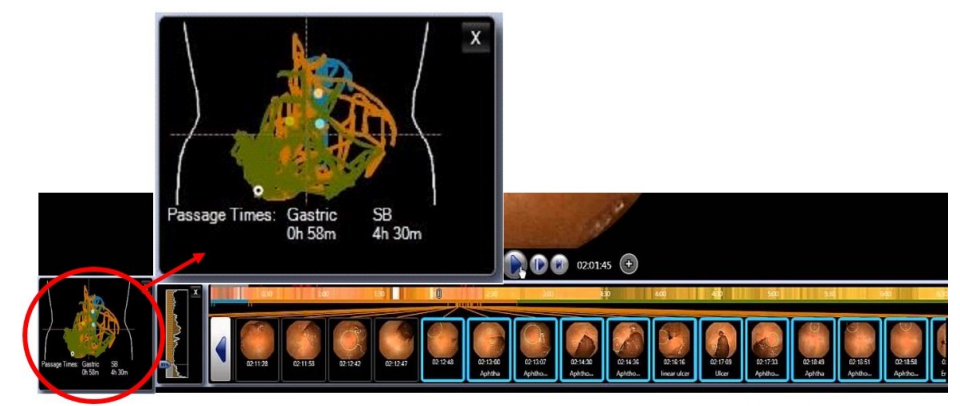

(b)

Figure 2.1: Sensors array (a) and movement pattern drawn by the software provided by Medtronic (b) [1]

Position estimation of the pill through this system is quite inaccurate so the doctor, looking at the drawn pattern (Figure 2.1(b)), cannot precisely locate detected abnormalities. This is of fundamental importance for their subsequent treatment either through surgery or through drug delivery.

\subsection{UWB for next-generation WCE}

Based on the IEEE Standard 802.15.6-2012 for Wireless Body Area Networks (WBANs) [10], the currently used frequency band for medical in-body devices, and therefore for capsule endoscopy, is the MICS band which is specified between $402 \mathrm{MHz}$ and $405 \mathrm{MHz}$. This band offers good penetration of the signal through the biological tissues. However, the maximum bandwidth is limited to $300 \mathrm{kHz}$ resulting in a data rate up to $500 \mathrm{kbps}$, which is not sufficient to transmit high quality images, as for WCE applications [11].

Several studies take into considerations the $2.4-2.5 \mathrm{GHz}$ Industrial, Scientific and Medical (ISM) frequency band for future WCE systems [12], [13]. This band is supporting the fast growth in various short range communication services such as Bluetooth, Zigbee, and Wireless Local Area Networks (WLANs). Additionally, the possibility to use the band from $2.4835 \mathrm{GHz}$ to $2.5 \mathrm{GHz}$ for Low Power Active Medical Implants (LP-AMI) has been considered by the European Telecommunications Standards Institute (ETSI) [14].

A drawback of the ISM band is the lack of any protection against interference from other communication services in the same band [15].

Recently [16], UWB and Impulse Radio Ultra-Wideband (IR-UWB) have been attracting attention as potential candidates for next-generation capsule endoscopes. In the United States (US), UWB technology was defined by the Federal Communications Commission (FCC) in 2002 as any wireless system 
occupying a bandwidth equal to or higher than $500 \mathrm{MHz}$ (absolute bandwidth) or a fractional bandwidth larger than the $20 \%$ of the carrier frequency (relative bandwidth) [17]. The FCC approved the unlicensed use of this technology in the electromagnetic spectrum which covers the frequency range from 3.1 to 10.6 GHz. In contrast to US, in Europe, regulation of UWB devices in the same frequency range was delayed. The first European Commission's decision was adopted in March 2006, ECC/DEC/(06)04 [18]. Several supplementary regulatory provisions to $\mathrm{ECC} / \mathrm{DEC} /(06) 04$ were provided in the following years till the latest one, 2014/702/EU [19] in 2014. For the European Commission, UWB was defined as a technology for short-range radio communication, involving the intentional generation and transmission of radio frequency energy that spreads over a frequency range wider than $50 \mathrm{MHz}$, which may overlap several frequency bands allocated to radio communication services [20].

Ultra-Wideband can support high-data-rate due to its large bandwidth, low power consumption, miniaturization capabilities and simple transceiver architecture [21].

UWB communication can achieve data transmission rates equal to or higher than 100 Mbps for electronic pill applications [22], [23]. The maximum allowable (both in Europe and in US) spectral density of $-41.3 \mathrm{dBm} / \mathrm{MHz}$ means that UWB transceivers, especially Impulse Radio (IR), can have extremely low power consumption and their radio emissions will not represent a significant source of electromagnetic interference to other medical equipment or devices operating in the vicinity. In addition, further miniaturization of electronic pills, including small antenna sizes can be attained with high frequency technology $[24]$.

Nevertheless, the high power loss caused by the large conductivity of biological tissues limits the application of UWB band. As a matter of fact, the lowest part of the UWB spectrum (3.1-5.1 GHz) is being considered in literature for in-body applications, due to the unaffordable signal attenuation above this frequency range [15]. It is of fundamental importance to investigate the wave propagation mechanism and characteristics in the UWB frequency band of interest.

\subsection{WCE localization techniques}

In recent years, different approaches for WCE localization have been investigated. These technologies can be divided into those using magnetic field or inertial systems [25], [26], those using image processing techniques [27], [28], [29], and those based on Radio Frequency (RF) signals [30], [31].

In magnetic sensing based techniques, a magnet is inserted into the WCE and the capsule is located by measuring the magnetic field [25] through several magnetic sensors set outside of the body. The intensity of the field is related 
to the magnet's three-dimensional location and two-dimensional orientation. Thus, at least five sensors are required to provide the field intensity to solve five simultaneous equations. As the human body has a magnetic permeability very close to non ferromagnetic materials such as air and water, therefore having very little influence on the magnetic field, it is possible to achieve high tracking accuracy. Nevertheless, this technique increases the weight and size of the WCE and the magnetic field used for localization might be interfered by the external magnetic fields used for other applications such as Magnetic Resonance Imaging (MRI) systems. Another option could be the insertion of radiation opaque material into the WCE and trace the location of the capsule using $\mathrm{X}$-ray or computed tomography (CT) scan [2]. However, continuous imaging using X-ray or CT scan is very expensive and it could harm the patient's health.

Employing the RF signal used for image transmission to also locate the capsule endoscope constitutes an efficient and low cost solution that does not increase the complexity of the capsule, thus allowing battery power saving. RF-based localization inside the human body is an evolution of RF technology applied to indoor localization. Therefore, in literature, same techniques used for indoor positioning are being investigated and adapted for WCE localization. Classical RF approaches consist, as a first step, in the estimation of one or more location dependent parameters, such as RSS [32], ToA [32], [33], Time Difference of Arrival (TDoA) [33], Phase Difference of Arrival (PDoA) [34], [35] or AoA/Direction of Arrival (DoA) [33], [36], [37]. Then, in a second step, the collected parameters are used to estimate the capsule's position through triangulation, trilateration or multilateration method [38]. Triangulation is the process of determining the location using angles estimation from three (2D) known reference points (Figure 2.2(b)), employing trigonometric properties.

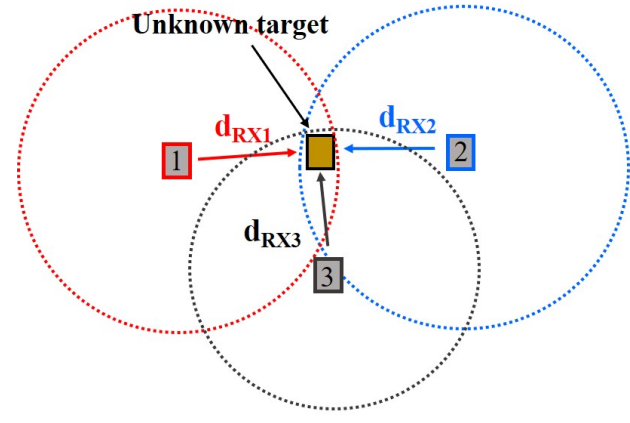

(a)

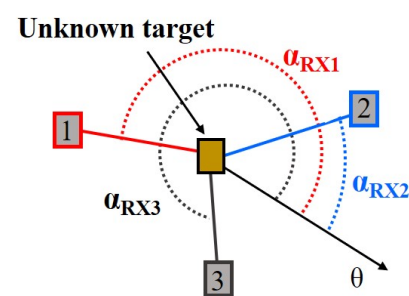

(b)

Figure 2.2: Localization using trilateration (a) and using triangulation (b) 
Trilateration (Figure 2.2(a)) and multilateration estimate the unknown target position as the intersection of three or more circles (2D) or spheres (3D) having radius equal to the estimated distance between the reference point and the unknown target to locate.

RSS-based localization technique exploits the attenuation of the received signal strength with distance from the source. The relationship between the received power, $P_{r}$, and the distance from the transmitter to the receiver can be expressed by a logarithmic path loss (also defined as system loss [39]) model, which is the most commonly proposed in literature [40], as:

$$
P_{r}(d B)=P_{t}-P L\left(d_{r e f}\right)-10 n \log _{10} \frac{d}{d_{r e f}}+S\left(d>d_{r e f}\right)
$$

where $d$ is the distance between transmitter and receiver, $P_{t}$ is the transmit power, $P L\left(d_{r e f}\right)$ is the path loss at the reference distance $d_{r e f}, n$ is the path loss gradient, determined by the propagation environment and $\mathrm{S}$ is the shadowing. From Equation 2.1 the distance among the capsule and the receiver, also referred as ranging distance, can be roughly estimated.

RSS-based localization technique is a commonly employed approach due to its simplicity and less sensitivity to bandwidth limitations [32], [41], [42]. However, the location estimation heavily depends on the accuracy of the path loss model of the propagation channel.

ToA-based method uses the one-way propagation delay $(\tau)$, also known as ToA, of the direct path signal in order to estimate the ranging distance, $d$, between the capsule and a receiver located above the body surface as given by:

$$
d=\frac{c}{\sqrt{\varepsilon_{r}}} \tau
$$

where $c$ is the speed of the light in free space and $\varepsilon_{r}$ represents the relative permittivity of the tissue that the signal is going through. A mathematical model of the variation of $\varepsilon_{r}$ is also necessary as the relative permittivity changes within the different types of tissue and with frequency if considering UWB communication system. Moreover, due to the inhomogeneous nature of the in-body environment the direct path signal is not always the strongest. Missed direct path errors, i.e. when a reflected signal path is declared to be the direct path, heavily affect position estimation of the capsule. In conclusion, time based techniques can provide highly accurate localization but they require a sharp pulse with wide bandwidth and strict time synchronization in order to obtain the desired precision.

Based on ToA technique, TDoA, two or more reference nodes are allocated to measure the received signals. After collecting the arrival time of received signals, time difference $(\Delta \tau)$ is derived by doing a simple subtraction. The evaluated $\Delta \tau$ is then used in Equation 2.2, instead of $\tau$, to have a first estimate of 
the distance. The difficulties of applying TDoA techniques are synchronization requirement and cooperation among all allocated nodes.

Phase of Arrival (PoA) gains its popularity in recent years because of the spread of Radiofrequency Identification (RFID). PDoA, the localization technique that develops from PoA, is also considered feasible to be applied in WCE localization although is still facing some vital challenges such as phase ambiguity and phase bias when penetrating different mediums.

Assume having two RF signals at different operation frequencies $f_{1}$ and $f_{2}$, then their respective phases are given by:

$$
\begin{aligned}
& \varphi_{1}=2 \pi\left(\frac{d f_{1}}{c / \sqrt{\varepsilon_{r}}}-n\right) \\
& \varphi_{2}=2 \pi\left(\frac{d f_{2}}{c / \sqrt{\varepsilon_{r}}}-n\right)
\end{aligned}
$$

where $d$ is the distance between transmitter and receiver, $c$ is the speed of the light in free space and $\varepsilon_{r}$ represents the relative permittivity of the tissue that the signal is going through and $n$ gives the phase ambiguity. From Equations 2.3 and 2.4 it is possible to derive the expression of $d$ as:

$$
d=\frac{c}{2 \pi} \frac{\varphi_{2}-\varphi_{1}}{f_{2}-f_{1}}=\frac{c}{2 \pi} \frac{2 \pi\left(f_{2}-f_{1}\right) \tau}{f_{2}-f_{1}}=c \cdot \tau
$$

AoA-based method, sometimes referred as DoA uses the angle/direction of incidence at which signals arrive at the receiving sensors to estimate the location of the transmitting source. To gather AoA information directive antennas or multiple antennas need to be implemented at each receiving sensor. Moreover orientation (azimuth and elevation angle) is needed to make use of AoA information. To this aim receivers could be equipped with a digital compass [43]. Nevertheless, DoA/AoA estimation algorithms are complex and their performance relies on several parameters such as the position, the implemented number of antennas, the antennas spacing and directivity etc. This needs to be taken into account when designing the receiving system [36].

Through one of the aforementioned techniques, a set of ranging estimates, which defines multiple circles (2D) or spheres (3D) whose intersection is the location of the unknown target (Figure 2.2(a)), can be obtained. The coordinates of the unknown target can be evaluated using linearized approaches as in [44], [45] or minimization algorithms such as Powell's [46], Newton's [47] or Levenberg-Marquardt method [48], [49]. In this thesis both, linear and non linear, methods are applied. The linear approach consists in linearizing the system of spheres $(3 \mathrm{D})$ or circles $(2 \mathrm{D})$ equations by subtracting the coordinates of the $\mathrm{j}$ th receiver, taken as reference [45]. This method reduces the degree of the equations system and convert the problem in a easier one, in terms of 


\subsection{RF localization at UWB frequencies}

computational complexity. Using this approach, at least three receivers are needed for $2 \mathrm{D}$ positioning and at least four for $3 \mathrm{D}$ positioning.

The non linear method consists in the minimization of the sum of the squares of the errors on the distance and it usually gives more accurate results than linear methods, despite a higher computational complexity [44].

More details regarding how both approaches were implemented in this dissertation are given in Section 3.3.2.2 and Section 3.3.2.3.

Since each individual approach is affected by measurement errors, hybrid localization methods could help improving the localization accuracy. These schemes consist in the fusion of more than one of the above described distancedependent parameters. Next, the hybrid scheme which uses RSS and AoA measurements is introduced as it is one of the techniques applied in this thesis. RSS/AoA approach is widely used in Wireless Sensor Networks [50], [43], [51] whereas for capsule endoscopy localization, no studies are available in literature. Range measurements can be obtained from RSS approach while angle measurements from AoA method. A set of range measurements defines multiple circles (2D) or spheres (3D) whose intersection is the location of the unknown target. Equivalently, a set of angle measurements define direction lines whose intersection is the area or space where the unknown target is located. When both, distance and angle, informations are available, the intersection area or space could be reduced and the localization accuracy of the unknown target improved.

Due to the non-homogeneity and severe attenuation of the RF signal propagating through different body tissues RF localization systems often end up providing discontinuous and scattered estimations with unacceptable amount of error. One way to enhance the performance of RF localization is to combine the motion information of the capsule by employing a data fusion algorithm such as Kalman filter or particle filter. In literature, there has been a trend to extract motion parameters from image sequence to improve the accuracy of RF localization. This class of algorithms is known as video-based Simultaneous Localization and Mapping (SLAM) algorithms [52]. In WCE applications it is possible to reconstruct motion information of the capsule from video stream, since the endoscopic capsule continually takes pictures with very short time interval (two-six frames/sec). Based on the motion model extracted from images a Kalman filter is then used to predict the position of the capsule and to obtain feedback from the RF measurements to correct the position estimations.

\subsection{RF localization at UWB frequencies}

As mentioned in Section 1.3, RF localization techniques for capsule endoscopy evolve from indoor positioning. However, in this case, the presence of the body tissues, instead of air, is even more problematic for the localization procedure, 
specifically in the Ultra-Wideband frequency band. In fact, the human body consists of different types of tissue each having its own electromagnetic properties. Particularly, permittivity and conductivity vary over the different tissues and present values much higher than those of the air. Moreover, these electromagnetic properties are frequency-dependent. As a consequence, the RF signal at UWB frequencies suffers from frequency-dependent attenuation and severe multipath conditions which makes ranging distance estimation very challenging. Despite these issues, radio frequency constitutes an optimal approach in order to keep the hardware of the capsule simple and to possibly reduce its battery consumption.

At present, performance analysis of RF-based localization techniques in the MICS frequency band are widely available in literature [53]. On the contrary, studies conducted in the UWB frequency band are quite limited and mainly based on software simulations using digital human models. Localization results through RF-based signals and compressive sensing are obtained in [41] using Computer Simulation Technology (CST) simulator with Finite Integration Technique (FIT) solver in 1-3 GHz and 3-5 GHz frequency bands. Best performance showed a mean localization error of $40 \mathrm{~mm}$. RSS-based approach is investigated in [54], through FIT simulator, in 1-6 GHz frequency band, showing a localization accuracy in the $\mathrm{cm}$ range. Authors in [55], address the crucial problem of ranging with UWB signals inside the human body using X (Window System) Finite Difference Time Domain (XFDTD) software platform, in 3.4-4.8 GHz frequency range. Ranging error (Root Mean Square Error (RMSE)) between $0.2-1.4 \mathrm{~cm}$ for antenna distances up to $6 \mathrm{~cm}$ was obtained with ToA approach, using the strongest path of arrival. Measurements campaign at $3-10 \mathrm{GHz}$ using a homogeneous phantom model to investigate the influence of body tissue on the accuracy of ToA-based ranging technique is presented in [56]. Besides possible inaccuracy of the UWB phantom model used, homogeneous phantoms only emulate one human tissue therefore they cannot model with sufficient accuracy the complex human body scenario. 


\section{Chapter 3}

\section{Scenarios and Methodology}

This chapter presents the considered scenarios and methodologies used to perform localization. The first section explains the application scenario used to emulate the real WCE procedure. The second section describes how data were collected through laboratory measurements, simulations and in vivo experiments. Finally, the third section is devoted to explain the implemented algorithms used to perform one-dimensional, two-dimensional and three dimensional positioning, respectively.

\subsection{Application Scenario}

As highlighted in Section 2.1, currently the capsule endoscope is locate through a sensors array placed on the patient's abdomen, shown in Figure 3.1, which receives the transmitted data from the pill during its movement along the Gastrointestinal (GI) tract.
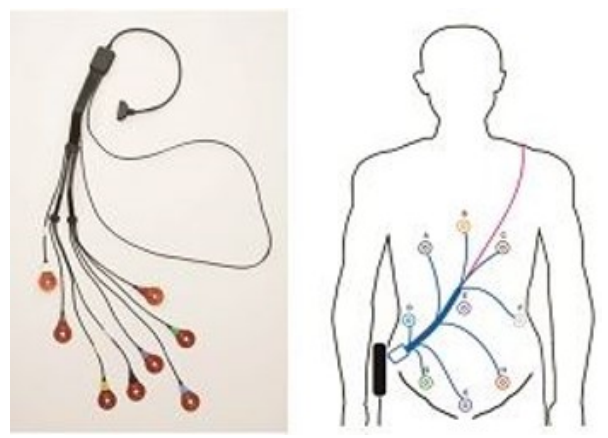

Figure 3.1: Sensors array provided by Medtronic [1] 
The information collected by the sensors is then processed off-line by the software provided to the hospitals in order to visually draw the movement pattern (Figure 2.1(b)) of the capsule travelling along the GI tract, depending on the landmark chosen by the physician.

With the aim of reproducing the real in-body to on-body WCE scenario depicted in Figure 3.1 for localization purposes, laboratory measurements, simulations and in vivo experiments, detailed in the following sections, were performed using, as transmitting in-body source, an UWB patch antenna, specifically designed to operate inside the human body, since there are currently no capsule endoscopes operating at these frequencies. Depending on the measurement campaign, several different UWB receiving antennas, specifically designed to operate on the surface of the human body were used. Specifically, the UWB receiving antenna was placed in different on-body locations in order to emulate the sensors array (Figure 3.1) currently used in capsule endoscopy procedure. The collected data were then processed off-line in order to locate in one-dimension, two-dimensions and three-dimensions the in-body antenna, as it will be explained in Section 3.3.

\subsection{Data Collection for RF Localization}

In this section the different approaches used to collect data to perform localization are presented. To this aim, three main methodologies were employed [2]: software simulations, experimental laboratory measurements and in vivo experiments. In software-based simulations, digital human models which include internal organs and blood flow are employed to emulate the electromagnetic properties of the human body. Many commercial software e.g., CST Microwave Studio, ANSYS High Frequency Structure Simulator (HFSS), are available for this scope. Despite the complexity and the high computational times, software simulations are easy to perform and always a good alternative to obtain data for localization purposes. Laboratory measurements involve tissue-equivalent liquids, called phantoms, which imitates the electromagnetic properties of the human tissues. In literature, phantom-based measurements gained popularity in the latest years, as an alternative to animal experimentation. However, accurate phantom models, tuned in the frequency band of interest and dedicated testbeds are required to properly emulate the WCE scenario. Lastly, in vivo experiments are not easy to conduct due to ethical restrictions and the necessity of dedicated facilities and competent medical personnel. Nevertheless, in vivo measurements are the closest to the real case scenario because they take into account all the tissues, blood and internal movements that characterize living bodies. 


\subsubsection{Frequency Domain Channel Sounding}

Experimental measurements (in laboratory and in vivo) of the in-body propagation channel were carried out by means of a Vector Network Analyzer (VNA) based testbed. The VNA triggers a frequency sweeper to obtain the frequency response of the channel with a certain resolution (see Figure 3.2). The resolution is given by the selected bandwidth and the frequency points chosen for the sweep. The resolution in frequency impacts the time resolution of the impulse response measurement in time domain.

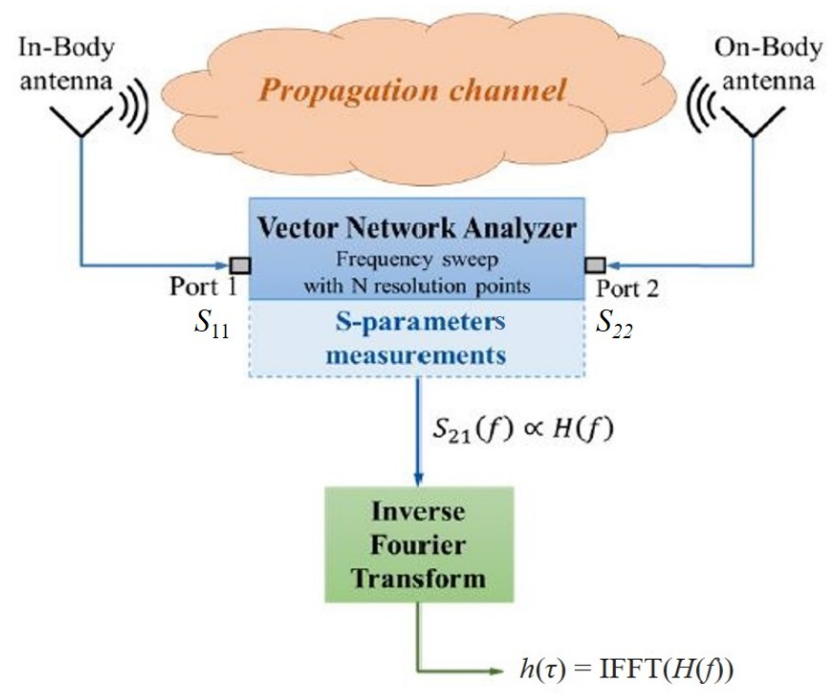

Figure 3.2: Experimental channel sounding of the IB2OB channel by using a VNA [2]

The VNA is used to measure and analyze signal behavior of S-parameters, or scattering parameters, which describe the input-output relationships between ports in a system, in frequency domain. At each frequency point, the S-parameters test set sends a known signal at port 1 and monitor the received signal at port 2. This procedure allows the VNA to determine what is known as complex frequency response of the channel $(H(f))$ or the forward transmission coefficient $\left(S_{21}\right)$. From $H(f)$, the channel impulse response $(h(\tau))$ is obtained by applying the Inverse Fast Fourier Transform (IFFT) to $H(f)$ within N resolution points.

Beside measuring the $S_{21}$ parameter, other scattering parameters such as the reflection coefficients are measured by the VNA. These parameters would be $S_{11}$ and $S_{22}$ in a two ports system. 


\subsubsection{Experimental Laboratory Measurements}

In this section, a brief description of the phantom models used to perform experimental measurements is given. Then, the remainder of the section is devoted to explain the three different measurement campaigns carried out at the Universitat Politécnica de Valéncia (UPV) facilities. The first one was conducted prior to the beginning of this thesis [57] using a homogeneous phantom model and it has been used to perform One-Dimensional (1D) localization. The subsequent two measurement campaigns were performed employing a homogeneous and heterogeneous phantom model, respectively, and used to perform Two-Dimensional (2D) localization.

\subsubsection{UWB phantom models}

Human experimentation for the development and testing of in-body channel propagation models is not allowed for obvious ethical restrictions. As a consequence, in order to reproduce the electromagnetic properties of the human body, tissue-equivalent liquids called phantoms started to gain popularity in literature. These phantoms are commonly a mix of ordinary ingredients, such as sugar, water and salt [58] and they are supposed to mimic the relative permittivity of the body tissues. Considering that the relative permittivity is frequency-dependent, phantoms are usually provided for specific and narrow frequency bands. UWB phantom models have also been provided in literature [59] but, besides the fact that they only mimic a limited number of tissues, in most of the cases they poorly approximate the relative permittivity of the specific human tissue in the whole UWB frequency range. Recently, researchers at UPV [60], [61], [62] have accomplished with this necessity. By means of acetonitrile solutions, human tissues with different content of water were properly mimicked in the $500 \mathrm{MHz}-26.5 \mathrm{GHz}$ frequency band, assuming the real properties of tissues being reported in [63].

The WCE scenario involves different human tissues, mainly colon, muscle and fat. As colon and muscle have similar permittivity, both have been considered as one single tissue, muscle, when performing heterogeneous phantombased measurements in Section 3.2.2.3. Moreover, muscle phantom is widely used in literature for in-body to on-body (IB2OB) communications studies.

Figure 3.3 compares the permittivity and conductivity of the muscle and fat phantom created at UPV with the real ones of human muscle and fat reported in literature [64]. Results show a good approximation of the electromagnetic properties of the tissues in the frequency range of interest (up to $8.5 \mathrm{GHz}$ ) for the first homogeneous and heterogeneous measurement campaigns described in Section 3.2.2.2.1 and Section 3.2.2.3, respectively. The muscle phantom is a mixture of Acetonitrile $(54.98 \%)$ and $\mathrm{NaCl}(1.07 \%)$ whereas the fat phantom is an emulsion of $86 \%$ of oil in water [65], where $1 \%$ of TX-100 was used as surfactant. 


\subsection{Data Collection for RF Localization}

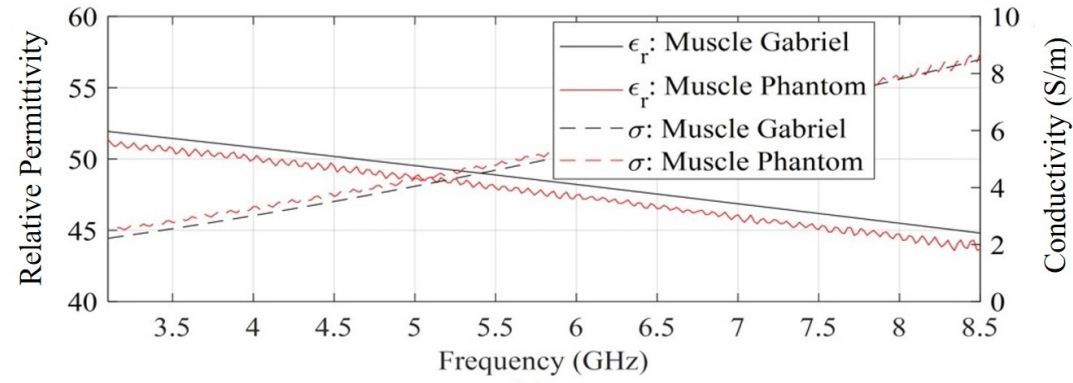

(a)

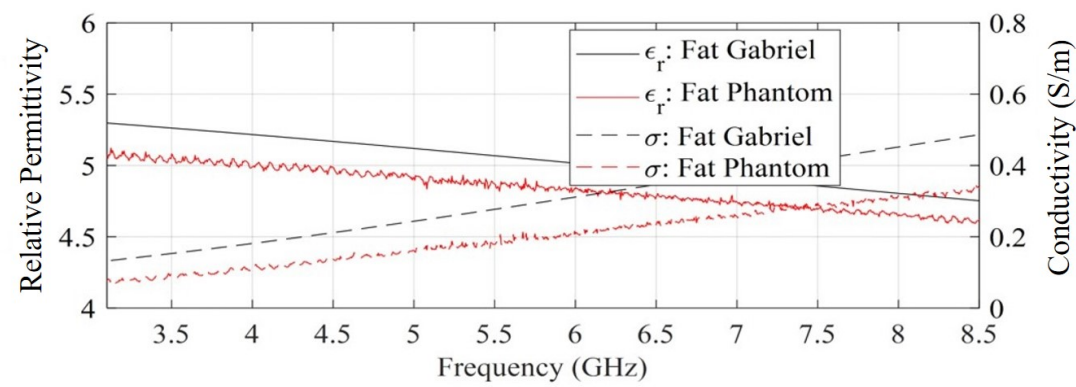

(b)

Figure 3.3: Permittivity and conductivity of muscle phantom (a) and fat phantom (b)

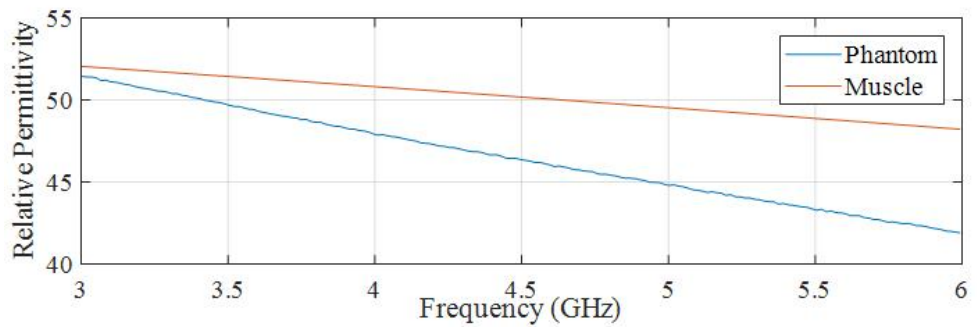

(a)

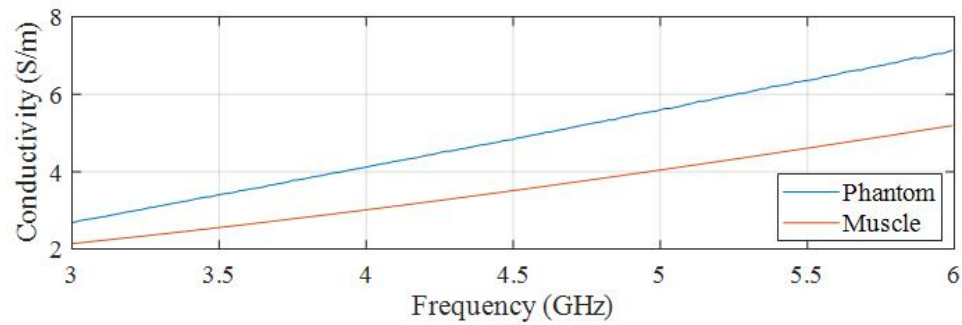

(b)

Figure 3.4: Permittivity (a) and conductivity (b) of muscle phantom made of sugar and water 
For the second homogeneous phantom-based measurements campaign, detailed in Section 3.2.2.2.2, a simple UWB phantom made of water and sugar, according to [66], was used to mimic the properties of muscle tissue in the 3-6 $\mathrm{GHz}$ frequency band. Figure 3.4 shows the measured permittivity and conductivity of the prepared muscle phantom model.

\subsubsection{Homogeneous phantom-based campaigns}

\subsection{Setup for 1D localization}

As mentioned at the beginning of Section 3.2.2, in order to start developing RF-based localization techniques data from a previously conducted measurements campaign [57] were considered. Figure 3.8(a) depicts the IB2OB scenario used for the measurements. To reproduce the propagation from inside to outside the human body the UWB muscle-tissue phantom presented in Figure 3.3(a) was used. The phantom was poured into a polypropylene box of size $30 \times 30 \times 15 \mathrm{~cm}^{3}$. Measurements were performed using, as in-body source, a small UWB patch antenna (Figure 3.5(b)), designed to operate inside the human body [67], since there are currently no ingestible wireless capsule endoscopes operating at these frequencies, as previously pointed out in Section 3.1 .

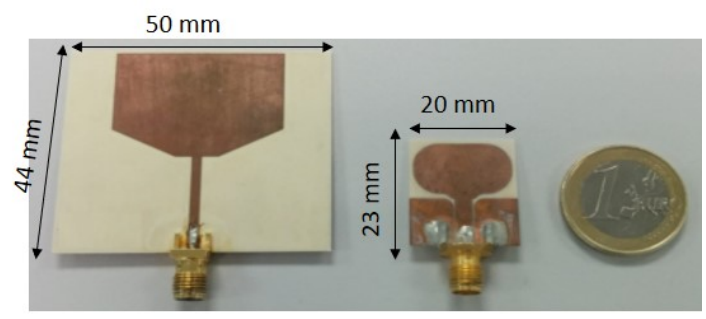

(a)

(b)

Figure 3.5: On-body (a) and in-body (b) antenna used in the measurements

The in-body antenna presents a quasi-omnidirectional radiation pattern in XZ-plane, as depicted in Figure 3.6(a) and it was designed to be matched to human muscle as shown in Figure 3.6(b) which reports its simulated reflection coefficient, $S_{11}$, versus its measured one in muscle tissue, prepared according to [66]. The receiving on-body antenna is an UWB patch antenna specifically designed to operate on the surface of the human body [68]. Figure 3.7(b) presents its simulated and measured reflection coefficient in the whole UWB frequency band. This antenna presents as well a quasi-omnidirectional radiation pattern in XZ-plane as shown in Figure 3.7(a). 


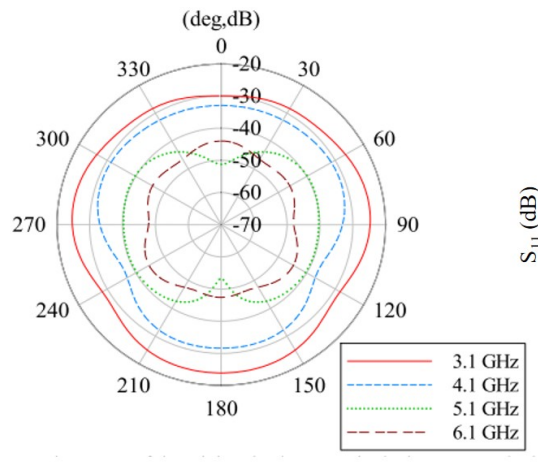

(a)

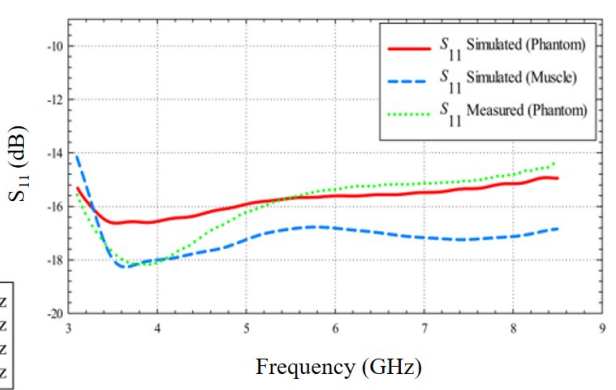

(b)

Figure 3.6: Radiation pattern in XZ-plane (a) and simulated vs measured $S_{11}$ of in-body (b) antenna

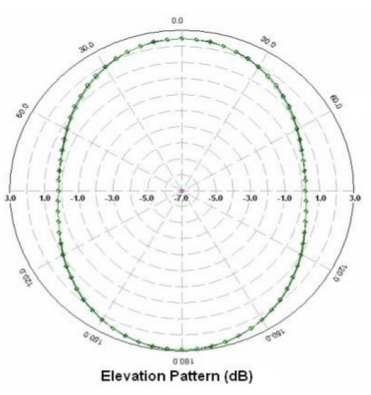

(a)

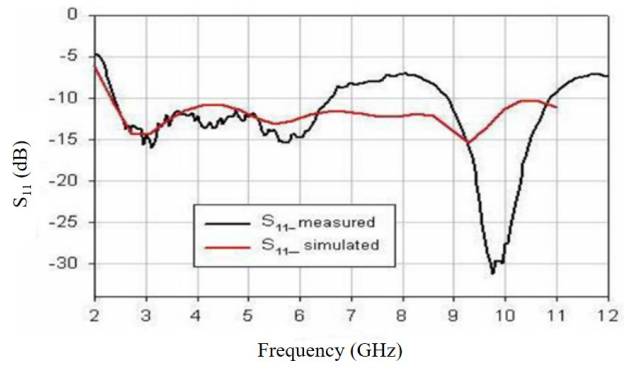

(b)

Figure 3.7: Radiation pattern in XZ-plane (a) and simulated vs measured $S_{11}$ of on-body (b) antenna

For the measurements conducted in this campaign, the receiving antenna was fixed in the center of the outer edge of the box, as shown in Figure 3.8(a). The in-body antenna was covered and isolated by a layer of latex rubber to avoid the physical contact with the liquid phantom, preventing a short-circuit of the printed board elements. Afterwards, it was attached to a $3 \mathrm{D}$ cartesian positioner (Figure 3.8(b)) and moved inside the muscle phantom in different spatial $(\mathrm{x}, \mathrm{y}, \mathrm{z})$ positions with step of $\Delta \mathrm{x}=\Delta \mathrm{y}=\Delta \mathrm{z}=1 \mathrm{~cm}$ in a xyz mesh of $16 \times 25 \times 3$ measurement points, as shown in Figure 3.8(a).

The positioner was used to speed up and automate the measurement process as well as to perform more accurate measurements. Three coils rotate a sheave system which moves a robotic arm across the xyz spatial directions. The coils are powered by two stepper motors. One step is equivalent to a movement of the robotic arm of $0.05 \mathrm{~mm}$. The error of this positioner is lower than 0.254 
$\mathrm{mm}$ per $\mathrm{cm}$ in each axis. The origin of coordinates or the reference position of the positioner is located in one of the corners as can be observed in Figure $3.8(\mathrm{~b})$.

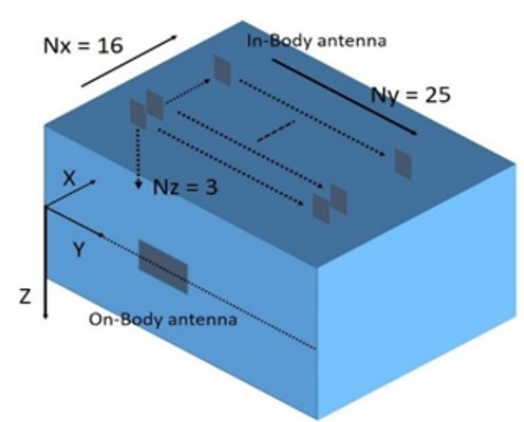

(a)

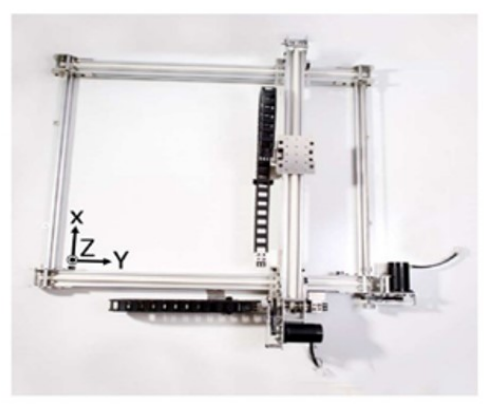

(b)

Figure 3.8: In-body to on-body measured grid (a) and XYZ cartesian positioner (b)

The forward transmission coefficient $S_{21}$ was measured by means of a VNA (see Section 3.2.1) in the 3.1-5.1 GHz frequency band, with 1601 resolution points, for each in-body to on-body antenna position. The noise threshold level for the on-body patch antenna was assessed to be $-90 \mathrm{~dB}$ (in terms of relative received power).

Due to the fact that only one position of the on-body antenna was considered in this measurements campaign, only one coordinate of the in-body antenna could be estimated at a time.

\subsection{Setup for $2 D$ localization}

Within a collaboration between the UPV and the Dresden University of Technology (TUD) experimental measurements were carried out at UPV facilities with the aim of comparing different on-body receiving antennas [69]. The setup was developed at UPV specifically for IB2OB measurements [70] and it is shown in Figure 3.9. The main novel components, besides the VNA and the $3 \mathrm{D}$ cartesian positioner described in Section 3.2.2.2.1, are an anechoic chamber and a magnetic tracker. All the equipment is software-driven by a laptop.

The anechoic chamber is used to isolate the interior from the external environment so that undesired contributions from the surroundings will not affect the measurements. The chamber has an internal volume of $1 \mathrm{x} 1 \mathrm{x} 1 \mathrm{~m}^{3}$ and the structure is built with wood of $22 \mathrm{~mm}$ width. The interior was coated with aluminum foil of $13 \mu \mathrm{m}$, which creates a sort of Faraday Cage for the frequencies under study (UWB), isolating the inside of the cage from the outside. The 
aluminum foil was then re-covered with flat absorbers made of polyurethane which are designed to attenuate at least $20 \mathrm{~dB}$ for frequencies above $1.3 \mathrm{GHz}$.
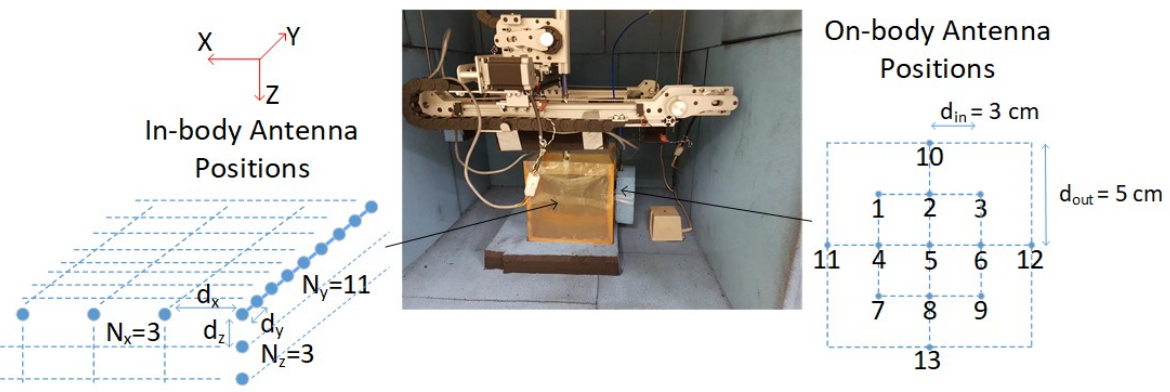

Figure 3.9: Measurement setup and in-body to on-body measured grid points

The 3D magnetic tracker transmitter (Figure 3.10(a), element (2)), located inside the anechoic chamber, is the absolute reference system for localization purposes (Figure 3.10(b)). Through the generation of a magnetic field and the sensors (Figure 3.10(a), element (3)), attached to the in-body and on-body antenna, the distance between antennas as well as the $\mathrm{x}, \mathrm{y}, \mathrm{z}$ coordinates of each antenna can be precisely evaluated. It is important to point out that the effect of the magnetic sensors over the antennas was tested and proved to be negligible.

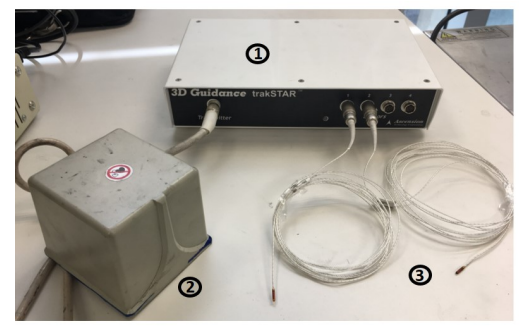

(a)

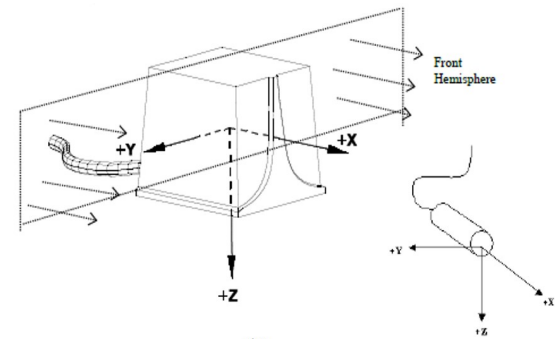

(b)

Figure 3.10: Magnetic tracker system (a): Electronic Unit (1), magnetic transmitter (2), magnetic sensors (3) and magnetic transmitter reference system (b)

A square plastic box, shown in Figure 3.9, was filled with the muscle phantom made of sugar and water presented in Figure 3.4. During the measurements the container was placed inside the anechoic chamber.

The in-body antenna used is the same described in the previous section and depicted in Figure 3.5(b). Two different on-body antennas were considered. The first one is the UWB quasi-omnidirectional patch antenna detailed in the 
previous section and showed in Figure 3.5(a). The second one is an UWB antipodal Vivaldi antenna fabricated at TUD (Figure 3.11(a)) and characterized by a directive radiation pattern [3] (Figure 3.11(b)). Figure 3.12 shows the measured reflection coefficients for the in-body antenna (inside the muscle phantom) as well as for both on-body antennas (on the external edge of the phantom container). As can be observed, all the antennas have a value lower than $-10 \mathrm{~dB}$ in the UWB frequency band of interest (3-6 GHz).

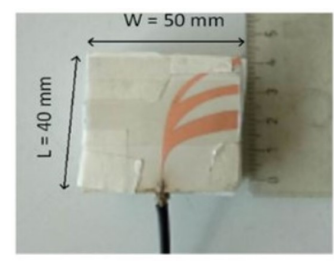

(a)

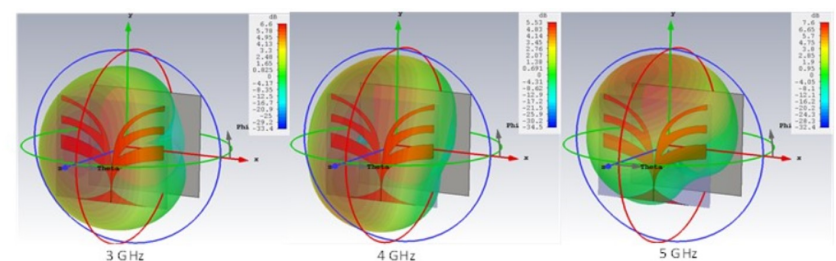

(b)

Figure 3.11: On-body Vivaldi antenna [3] (a) and its related radiation pattern (b)

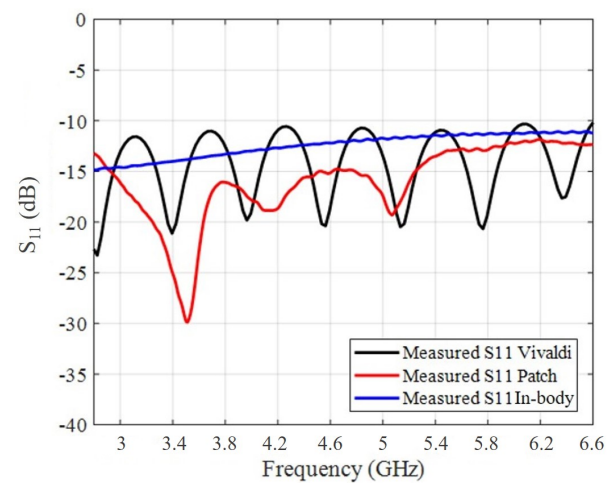

Figure 3.12: Measured reflection coefficient for the on-body Patch and Vivaldi antenna and for the in-body antenna

The on-body antenna was manually placed in YZ plane on the external edge of the container, in 13 different locations, as shown on the right side of Figure 3.9 , in order to emulate the sensors array used in real WCE applications (Figure 2.1(a)). The distance between on-body positions, also referred as receivers, in the internal grid $(\operatorname{Rx} 1$ to $\mathrm{Rx} 9)$ is $d_{i n}=3 \mathrm{~cm}$, while the distance between the external grid ( $\mathrm{Rx} 10$ to $\mathrm{Rx} 13)$ is $d_{\text {out }}=5 \mathrm{~cm}$. The in-body antenna was moved inside the muscle phantom with a spatial resolution of $(\mathrm{dx}, \mathrm{dy}, \mathrm{dz})=(3 \mathrm{~cm}, 1$ $\mathrm{cm}, 3 \mathrm{~cm})$ resulting in a total number of sample points per axis of $(\mathrm{Nx}, \mathrm{Ny}, \mathrm{Nz})$ 
$=(3,11,3)$. The same grid was repeated for each receiving on-body position to emulate 13 receivers receiving simultaneously. Moreover, for each receiver 5 snapshots of the measurements were taken to minimize the fluctuation due to the movement of the robotic arm while moving from one in-body position to another. The $S_{21}$ parameter was measured in the $3-6 \mathrm{GHz}$ frequency band with $\mathrm{N}=3201$ resolution points. The intermediate frequency and output power were set as at $3 \mathrm{kHz}$ and $8 \mathrm{dBm}$, respectively. Finally, the noise threshold level assessed for each antenna under these conditions was $-90 \mathrm{~dB}$ for the patch antenna and $-110 \mathrm{~dB}$ for the Vivaldi antenna.

As the magnetic sensors attached to the antennas are highly sensitive to small movements, for each snapshot, the position was calculated 100 times in order to minimize the errors. Then, the distance between antennas as well as their $\mathrm{x}, \mathrm{y}, \mathrm{z}$ coordinates were calculated by averaging the 500 collected measurements, for each in-body to on-body position.

Considering the setup depicted in Figure 3.9 only two coordinates of the in-body antenna ( $y$ and $z$ ) could be estimated as all receivers share the same $\mathrm{x}$-coordinates.

\subsubsection{Heterogeneous phantom-based campaign}

Experimental measurements, using a multilayer phantom-based setup were conducted at the UPV facilities. A novel two-layer phantom container (element 5 in Figure 3.13) has been designed to more precisely emulate the complex human body environment involved in WCE applications [70]. The container is made of Polyethylene terephthalate (PET) of $1.5 \mathrm{~mm}$ width and has an overall internal volume of $25 \times 25 \times 25 \mathrm{~cm}^{3}$ with two layers. Particularly, the layer of size $23 \times 25 \times 25 \mathrm{~cm}^{3}$ was filled with muscle phantom and the layer of size $2 \times 25 \times 25$ $\mathrm{cm}^{3}$ with fat phantom.

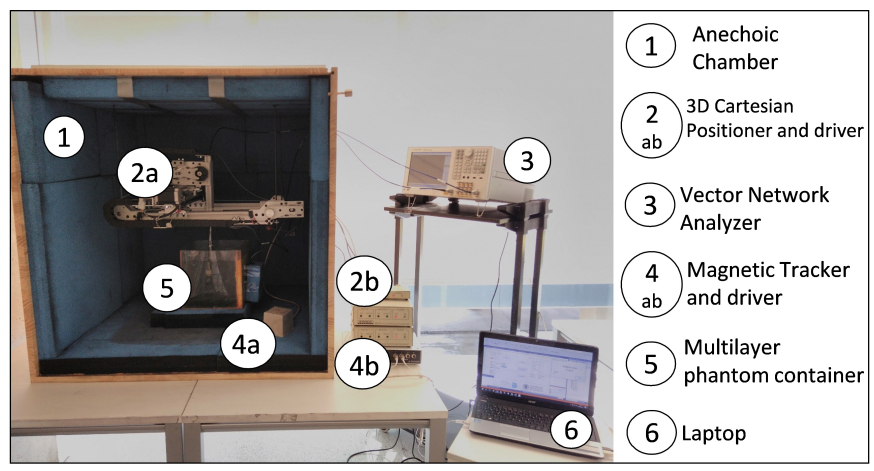

Figure 3.13: Multilayer phantom-based setup 
The phantoms used for the measurements are those described in Section 3.2.2.1, Figure 3.3.

The measurement equipment used is the same described in the previous section and the testbed is depicted in Figure 3.13. The in-body and on-body UWB patches antennas were the same ones detailed in the previous sections (Figure 3.5). Figure 3.14 reports the measured reflection coefficient for the in-body antenna (inside the muscle layer) and for the on-body antenna (on the outer edge of the fat layer). As can be observed, both antennas present a value lower than $-10 \mathrm{~dB}$ in the UWB frequency band of interest $(3.1-8.5 \mathrm{GHz}$ in this case).

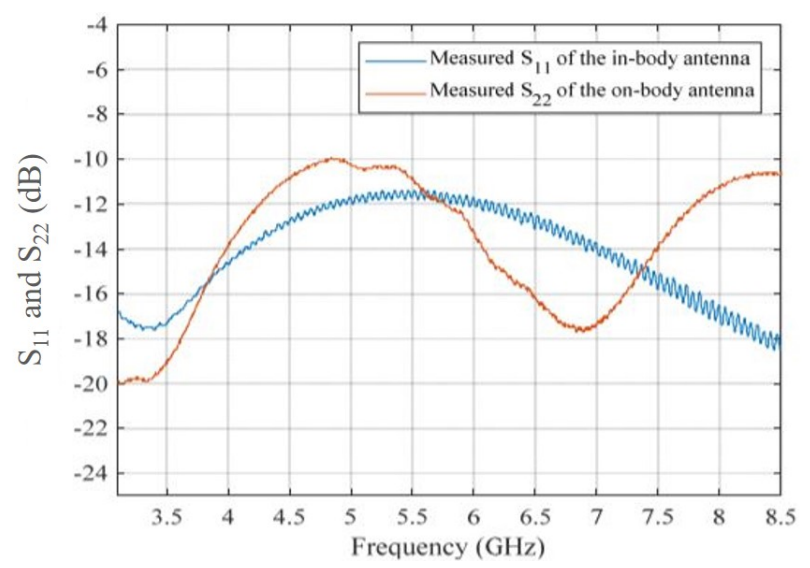

Figure 3.14: Measured reflection coefficient, for the in-body and on-body antenna

During the experimental campaign, the phantom container was placed inside the anechoic chamber and the in-body antenna, through the 3D Cartesian positioner, moved inside the muscle layer, in steps of $1 \mathrm{~cm}$ along $\mathrm{x}, \mathrm{y}, \mathrm{z}$ axis with a grid size of $(\mathrm{Nx}=12, \mathrm{Ny}=11, \mathrm{Nz}=2)$ as shown in Figure 3.15.

Five different positions, with a separation of $2 \mathrm{~cm}$, over the outer edge of the fat layer, as shown in Figure 3.15, were considered for the on-body antenna. For each location, the in-body antenna movement along $\mathrm{x}, \mathrm{y}, \mathrm{z}$ axis was repeated in order to emulate five on-body antennas receiving at the same time. For each in-body to on-body antenna position the $S_{21}$ parameter was measured in the 3.1-8.5 GHz UWB band, considering 3201 resolution points in frequency. The noise threshold level was assessed to be $-90 \mathrm{~dB}$. For each location, measurements were repeated five times (snapshots) with the aim of improving the Signal-toNoise Ratio (SNR). Then, the average $S_{21}$ was evaluated. 
Again, for each snapshot, the position was calculated 100 times and then, the distance between antennas as well as their $\mathrm{x}, \mathrm{y}, \mathrm{z}$ coordinates calculated by averaging the 500 collected measurements, per in-body to on-body position.

Considering the receivers configuration in Figure 3.15, only two coordinates of the in-body antenna, i.e., y and $\mathrm{z}$, could be estimated as all receivers share the same $\mathrm{x}$-coordinate.

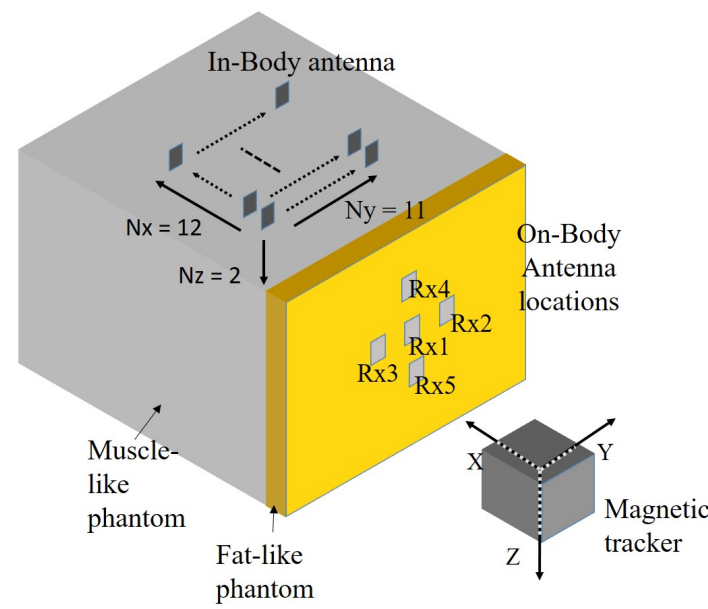

Figure 3.15: Measured grid points

\subsubsection{Software Simulations}

This section presents the software-based simulations, conducted at the UPV facilities, to collect data for RF localization.

Software simulations using the commercial software CST Microwave Studio were performed in the 3.1-5.1 GHz frequency band. The parameter $S_{21}$ was evaluated by the software through the Finite Difference Time Domain (FDTD) method. The abdominal part of a human female CAD model (Nelly) [71] has been used. This model mimics the permittivity of the human skin, fat and muscle and it was primarily used to compare the simulation results with those obtained by the multilayer phantom-based laboratory measurements, described in Section 3.2.2.3. Since the human muscle has permittivity similar to the small bowel/colon [64], this model has also been used to confirm the results obtained through in vivo experiments, presented in the following section. No internal organs as well as no blood flow were considered, in order to reduce the computational time of the simulations. Figure 3.16 shows the described 
model along with the in-body and on-body antenna positions considered in a first preliminary study.

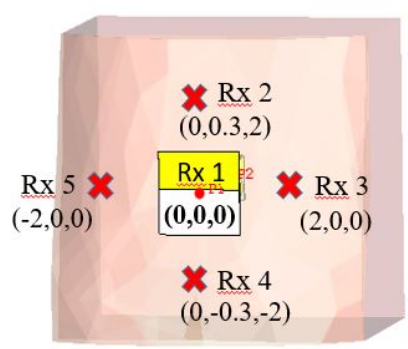

(a)

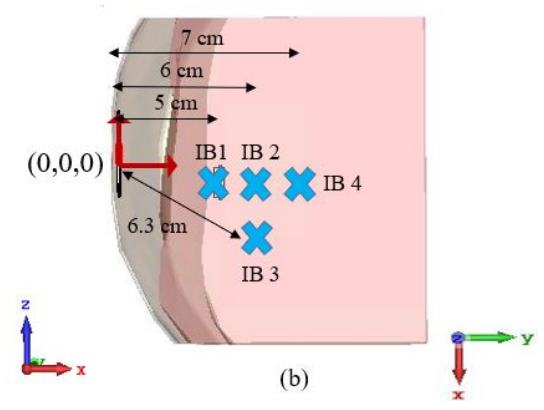

Figure 3.16: On-body (a) and in-body (b) antenna locations (top view)

The UWB in-body and on-body antenna used in the simulations (Figure 3.5) are the same employed for the laboratory measurement campaigns detailed in the previous section.

Measurements were performed by moving the in-body antenna in steps of 1 $\mathrm{cm}$ in four different locations along $\mathrm{x}$ and $\mathrm{y}$ axis inside the muscle layer (Figure $3.16(b))$. Five on-body antenna positions $(\operatorname{Rx} 1$ to $\operatorname{Rx} 5)$, with a separation of $2 \mathrm{~cm}$ along $\mathrm{x}$ and $\mathrm{z}$-axis and of $0.3 \mathrm{~cm}$ along $\mathrm{y}$-axis, were considered on the abdominal region of the CAD model (Figure 3.16(a)). As shown in Figure 3.16 , the center $(\mathrm{P} 1)$ of the on-body receiving antenna $\mathrm{Rx} 1$ is taken as the origin of the reference system used to evaluate the real and estimated positions of the in-body antenna.

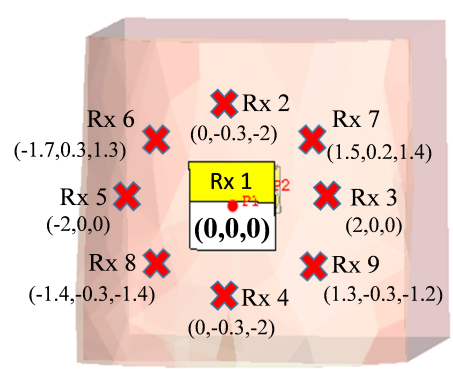

(a)

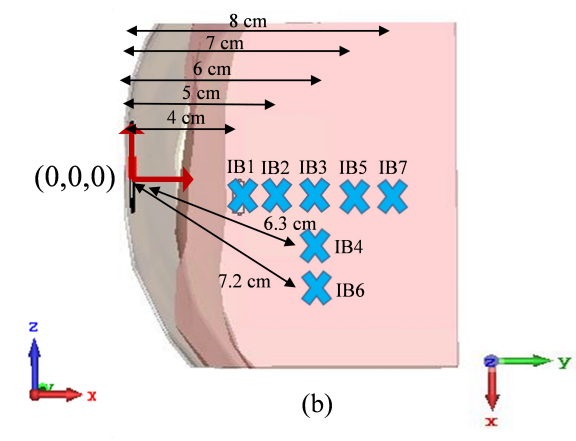

(b)

Figure 3.17: Supplementary simulations: On-body (a) and in-body (b) antenna locations (top view) 
In a second step, more simulations with the same setup were carried out to collect more data. Figure 3.17 shows the supplementary measured in-body and on-body positions. Three additional in-body locations, IB1, IB6, IB7 and four additional on-body positions, $\mathrm{Rx} 6, \mathrm{Rx} 7, \mathrm{Rx} 8, \mathrm{Rx} 9$, as depicted in Figure 3.17 , were considered.

\subsubsection{In Vivo Measurements}

Experiments in living animals are not easy to conduct as they are subject to ethical restrictions and extremely costly as dedicated facilities and a specialized medical team are required. Despite these difficulties, in vivo experiments, is the most realistic approach compared to laboratory measurements (controlled environment) and simulations (ideal environment) for in-body radio channel characterization and thus, for the testing of RF-based localization techniques. As a matter of fact, in vivo measurements are of high relevance in order to test developed localization algorithms in a scenario which is the closest to reality, as it includes breathing, movement of internal organs and blood flow (since the animal is alive) as it would be in real capsule endoscopy procedures with human beings. In this section, the three in vivo measurement campaigns carried out at the hospital La Fe, in Valencia are detailed. For comparison purposes, in vivo measurements were designed and conducted to resemble the multilayer laboratory measurements and simulations described in the previous sections. On this regard, it is important to mention that for the first two campaigns the orientation of the in-body and on-body antenna used in the experiments, as far as possible, was kept the same in order to better investigate the effect of the propagation channel on the localization performance.

\subsubsection{Living animal subjects}

In vivo measurements were conducted at the animal laboratory of the Hospital Universitari i Politécnic la Fe in Valencia, Spain, after approval by the Ethical Committee of Investigation of the hospital, under the protocol WIBEC 2015/0463. Pig subjects were chosen for the experiments due to the high similarities between swine and human anatomy. The animal subjects used for the three in vivo measurement campaigns were female pigs which weighed between 36 and $40 \mathrm{~kg}$, approximately.

\subsubsection{First experiment}

Same equipment (VNA, magnetic tracker, in-body and on-body antenna) described in Section 3.2.2.3 for the multilayer phantom-based measurement campaign was used. As the main scenario of interest for WCE applications is the GI tract, the in-body antenna was placed, through laparoscopy, in two different 
positions inside the abdomen of the porcine model, in order to be surrounded by either small bowel or colon or both. The first (Figure 3.18(a)) in-body position was surrounded by small bowel whereas the second one (Figure 3.18(b)) was surrounded by small bowel and colon.

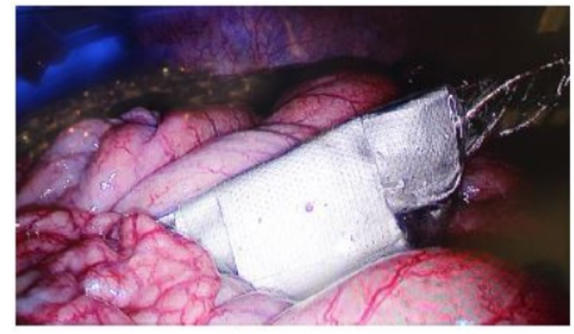

(a)

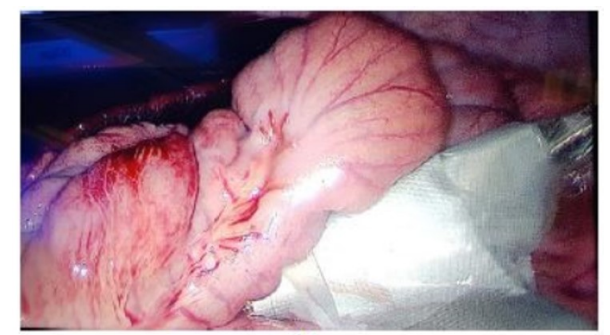

(b)

Figure 3.18: First (a), and second (b) measured in-body position

For each in-body location, the on-body antenna was placed on the abdomen of a porcine model, in direct contact with the skin (Figure3.19(a)), in thirteen different locations, as depicted in Figure 3.19(b).

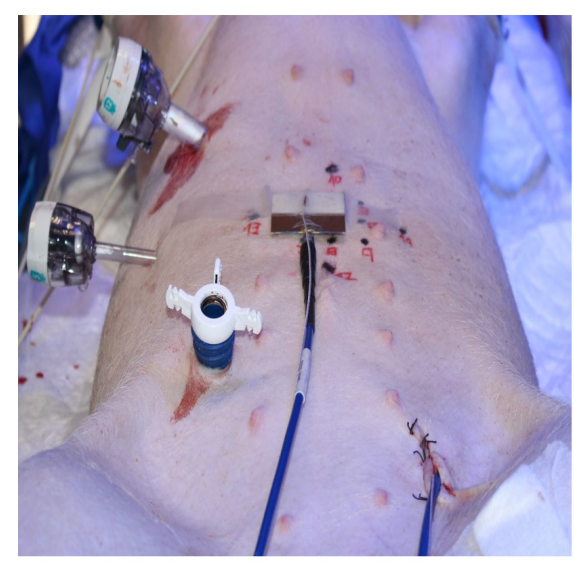

(a)

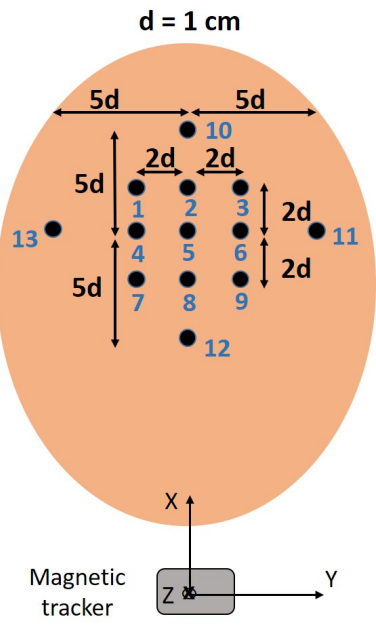

(b)

Figure 3.19: In vivo measurements for one on-body position (a) and measured grid for each in-body position (top view) (b)

Figure 3.20 and Figure 3.21 shows the reflection coefficients of the in-body and on-body antenna, respectively, for each measured in-body locations and 
for all the thirteen receiving positions. As can be observed, the reflection coefficient of the in-body and on-body antenna varies depending on the position of the antenna. The on-body antenna is matched within the frequency band of interest for all the thirteen measured locations per in-body position, since the $S_{22}$ coefficient is below or equal to $-10 \mathrm{~dB}$, as shown in Figure 3.21. The first (IB1) and second (IB2) in-body position in Figure 3.20 have different $S_{11}$ response, most likely because IB1 was surrounded by small bowel only and IB2 was surrounded by small bowel and colon. Anyway, the $S_{11}$ has a maximum value of $-7 \mathrm{~dB}$ (Figure 3.20(a)) and therefore the antenna is considered matched as well as in [72], where the antenna is considered matched for a value below or equal to $-6 \mathrm{~dB}$ for in vivo experiments.

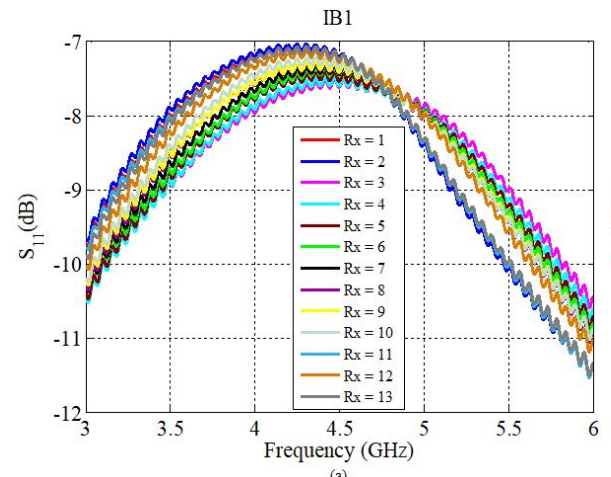

(a)

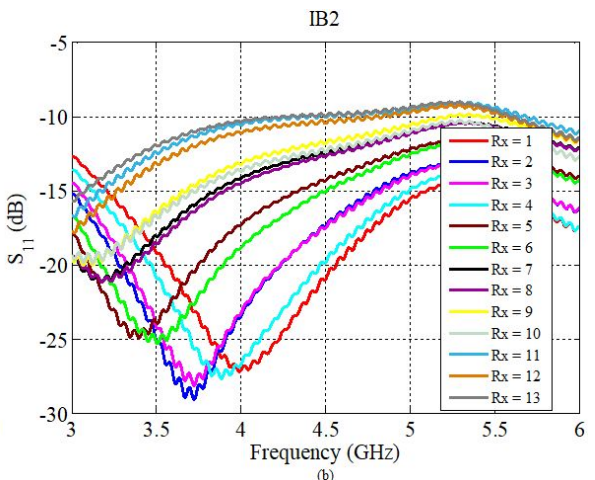

(b)

Figure 3.20: $S_{11}$ for the first (a), and second (b) measured in-body position
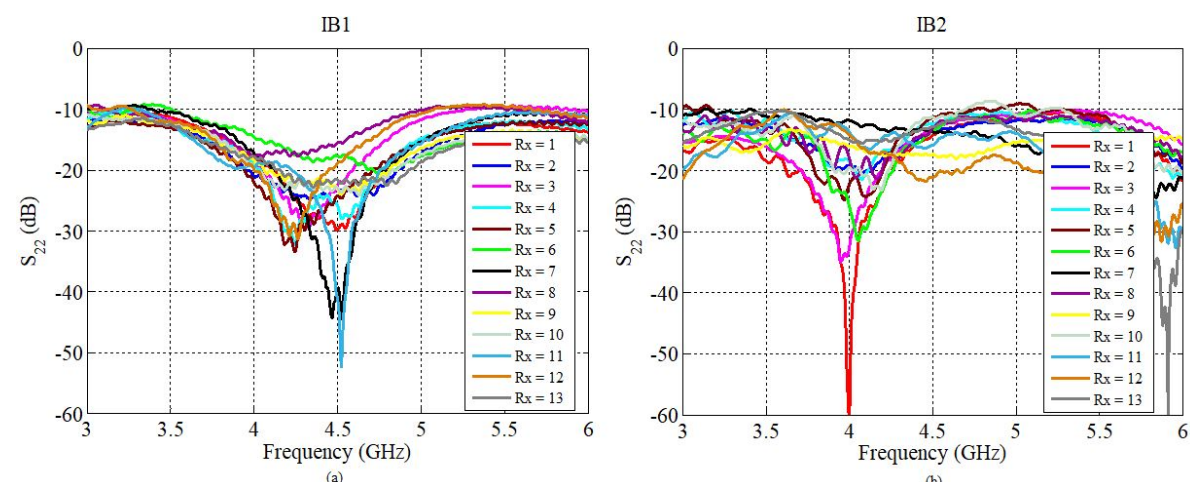

Figure 3.21: $S_{22}$ for the first (a), and second (b) measured in-body position

Measurements were taken in the $3-6 \mathrm{GHz}$ UWB frequency band, considering 1601 resolution points in frequency. Through the VNA, for each pair of in-body 
to on-body positions five snapshots of the channel $\left(S_{21}\right)$ were taken. The noise threshold level under these conditions was assessed to be $-90 \mathrm{~dB}$.

As for the laboratory measurements in the previous section, antenna separation distance and antenna coordinates were evaluated 100 times per snapshot by the magnetic tracker. Finally, averaging the collected measurements, $S_{21}$, antennas separation distance and antennas coordinates were evaluated.

\subsubsection{Second experiment}

Using the same equipment and methodology described in the previous section, in vivo measurements were conducted in the 3.1-5.1 GHz UWB frequency band, considering 2001 resolution points in frequency. Same in-body antenna used for the in vivo campaign detailed in the previous section was employed.

In this campaign, measurements were performed using three different on-body antennas, shown in Figure 3.22.

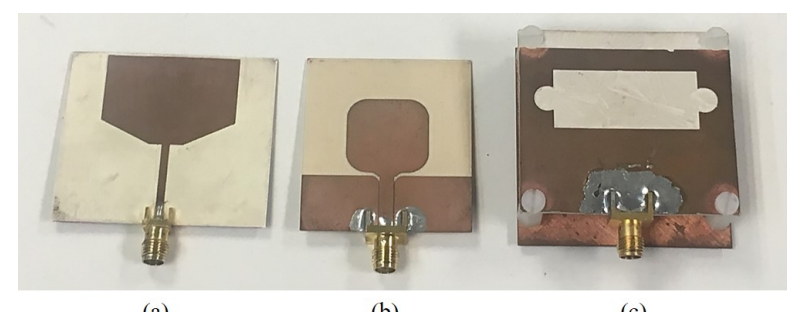

(a)

(b)

(c)

Figure 3.22: On-body antennas: First Patch antenna (a), Second Patch antenna (b), Directive antenna (c)

The first one (Figure 3.22(a)) is the same patch antenna used in the previous in vivo campaign (Figure 3.5(a)). The second one (Figure 3.22(b)) is an omnidirectional UWB patch antenna fabricated at UPV facilities as 'backup' of the first one. The third one (Figure 3.22(c)) is a directive slot patch antenna characterized by a reflecting plane designed to improve the penetration of the signal through the tissues [73], [74].

The in-body antenna was placed, through laparoscopy, in three different locations inside the abdomen of the porcine model, surrounded by either small bowel or colon or both. For each in-body position, the on-body antennas were placed, one at a time, in thirteen different locations on the abdomen of the swine as depicted in Figure 3.23.

Figure 3.24 shows the reflection coefficients of the in-body antenna, for each measured in-body locations and for all the thirteen receiving positions. As can be observed, the antenna is well matched within the frequency band of interest, for all measured in-body locations. 


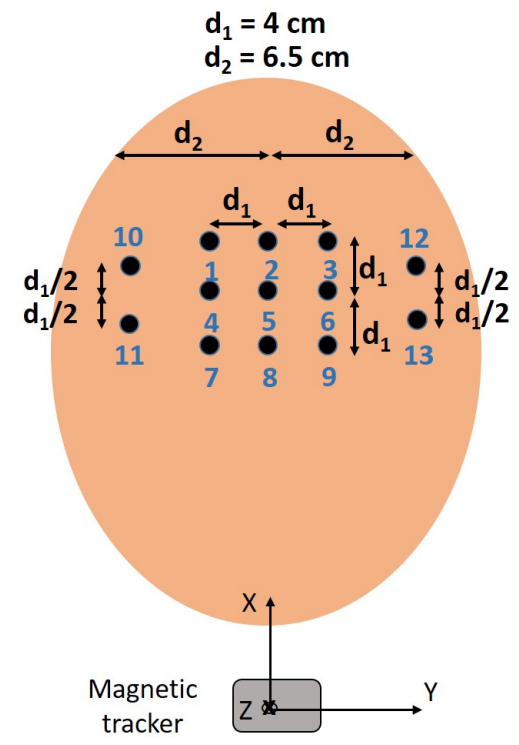

Figure 3.23: Measured on-body positions (second in vivo experiment)
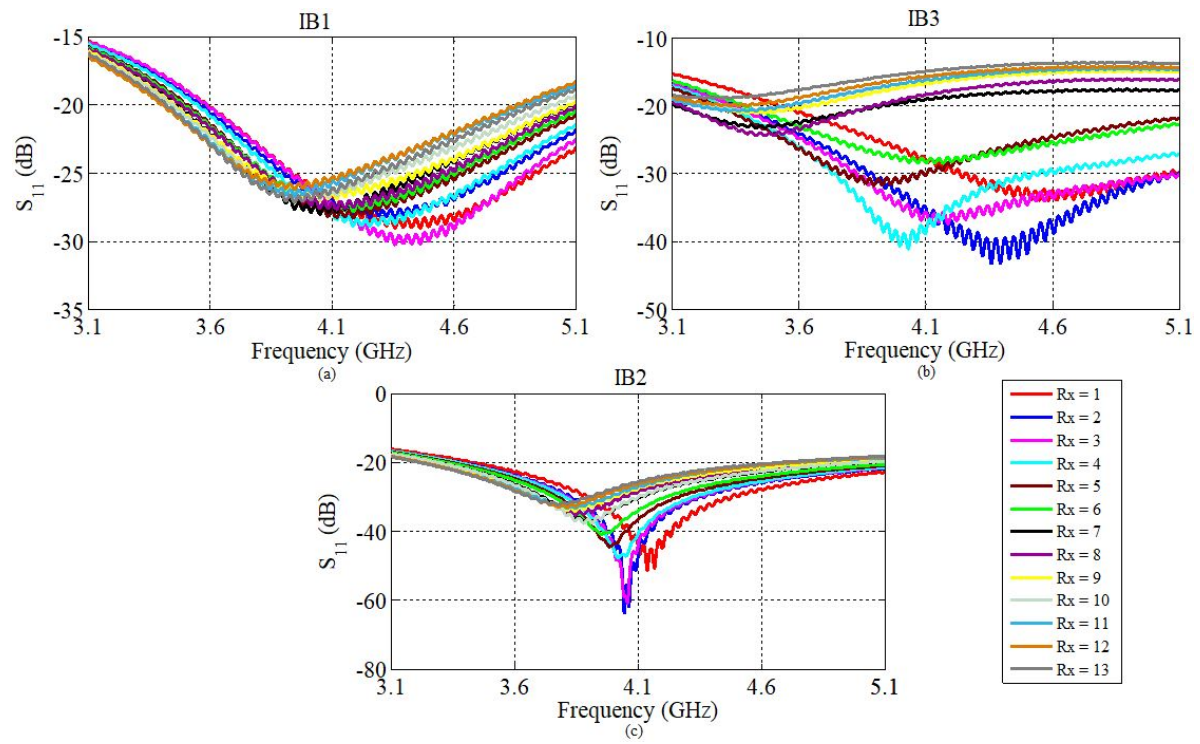

Figure 3.24: $S_{11}$ for the first (a), second (c) and third (c) measured in-body position 
Figure 3.25 reports the reflection coefficient of the on-body antennas for the third measured in-body position. The Patch 1 antenna has good matching within the considered frequency band. The directive and Patch 2 antenna are characterized by a slightly higher reflection coefficient, with a maximum value of $-6 \mathrm{~dB}$, which can still be considered acceptable [72]. Similar behavior was assessed for the first and second in-body locations.

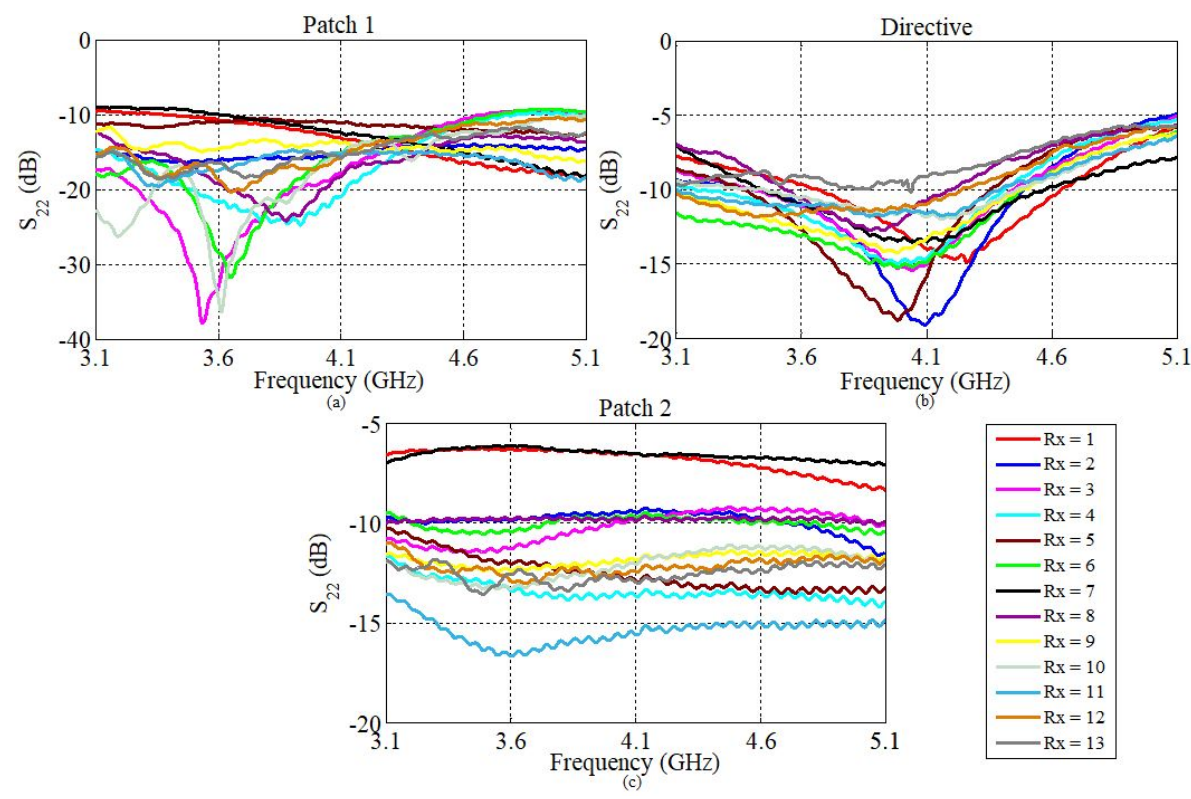

Figure 3.25: $S_{22}$ for the Patch 1 (a), Patch 2 (b) and Directive (c) antenna for the third measured in-body position

The $S_{21}$ parameter was measured five times per each in-body to on-body position. The noise threshold level was assessed to be $-90 \mathrm{~dB}$ for the Patch 1 and Patch 2 antenna and $-100 \mathrm{~dB}$ for the directive antenna, respectively.

Antenna separation distance and antenna coordinates were evaluated 100 times per snapshot by the magnetic tracker. Then, by averaging the collected measurements, $S_{21}$, antennas separation distance and antennas coordinates were evaluated.

\subsubsection{Third experiment}

This experiment was conducted during an experimentation week at the hospital La Fe, in Valencia, within the Wireless In-Body Environment (WiBEC) Innovative Training Network (ITN) with the aim of showing to the attendees 
how in vivo measurements are performed. Same equipment (including the inbody antenna) and methodology explained for the previous two in vivo campaigns were used. Four on-body antenna were employed in this experiment. The first two are the same patch and 'backup' patch antenna employed in the previous campaign and showed in Figure 3.22(a) and (b), respectively. The third and fourth antenna were manufactured at the UPV facilities for Body Area Network (BAN) applications and showed in Figure 3.26.

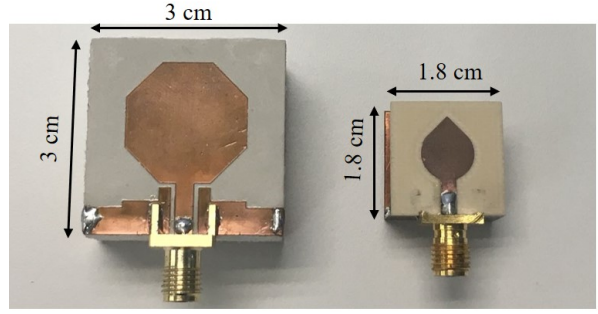

(a) (b)

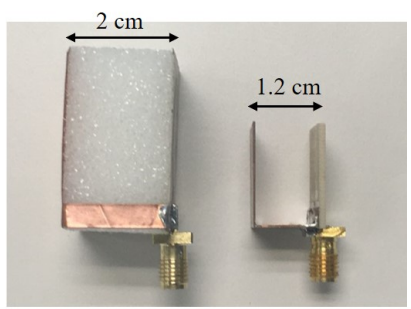

(a) (b)

Figure 3.26: Coplanar Waveguide (CPW) monopole (a) and tear-shape UWB monopole

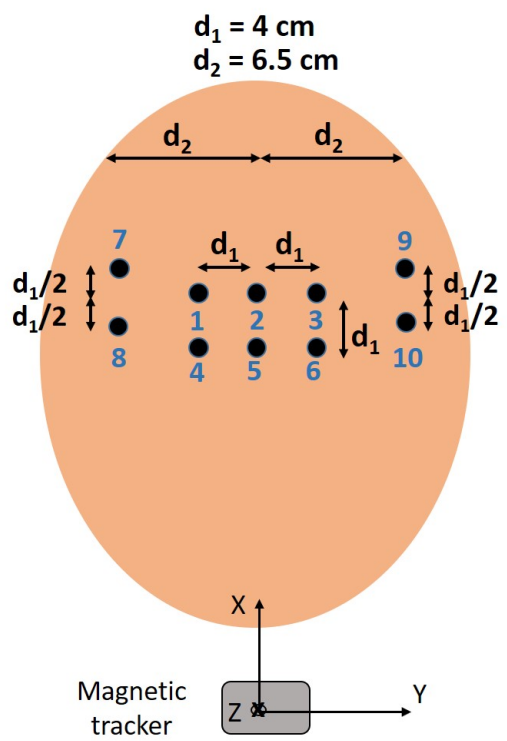

Figure 3.27: Measured on-body positions (third in vivo experiment)

The first antenna in Figure 3.26(a) is an UWB octagon Coplanar Waveguide (CPW) monopole [75] and the second one in Figure 3.26(b) is a tear-shape 
UWB monopole antenna [76]. Both were designed with a reflector plane to focuses the radiation of the monopole in the direction of the human body and enhance the gain. Measurements were performed in the 3.1-5.1 GHz frequency band, in the same way as for the previous in vivo campaigns. For each onbody antenna and in-body position the measured on-body locations were ten and they are shown in Figure 3.27. The noise threshold level was assessed to be $-90 \mathrm{~dB}$ for the Patch 1 antenna, $-95 \mathrm{~dB}$ for the Patch 2 antenna and - 100 $\mathrm{dB}$ for the CPW and tear-shape antenna, respectively.

Two in-body locations were measured. For the first in-body position, measurements were performed using as on-body antennas the Patch and the octagon CPW monopole antenna. For the the second in-body position, measurements were performed using the backup Patch and the tear-shape monopole antenna. Using the Patch 2 antenna only seven on-body positions, out of ten, were measured due to lack of time.

Figure 3.28 shows the reflection coefficients of the in-body antenna, for each measured in-body locations and for all the ten receiving positions. As can be observed, the antenna is matched within the frequency band of interest, for both measured in-body locations.
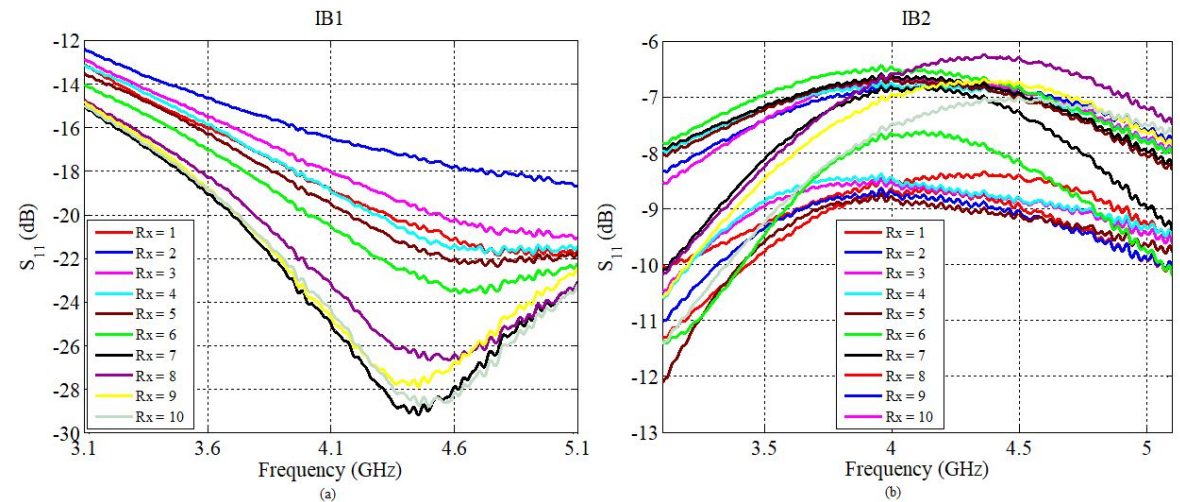

Figure 3.28: $S_{11}$ for the first (a) and second (b) measured in-body position

Figure 3.29 reports the reflection coefficient of the on-body antennas. All the antennas present a $S_{11}$ below $-6 \mathrm{~dB}$ within the considered frequency band except for Rx2 using the tear-shape antenna. This might be due to an antenna misplacement when measuring such on-body position.

It is important to point out that, in this experiment, the in-body antenna was placed inside the abdomen of the pig, at the surgeon's discretion, meaning that, in this case the orientation of the in-body and on-body antenna might have changed. For these measurements, the software controlling the magnetic 


\subsection{Offline RF-based Localization}

tracker was slightly changed in order to measure, besides their coordinates and distance, azimuth and elevation angles of the sensors attached to the antennas, with respect to the magnetic transmitter reference system (Figure 3.27).
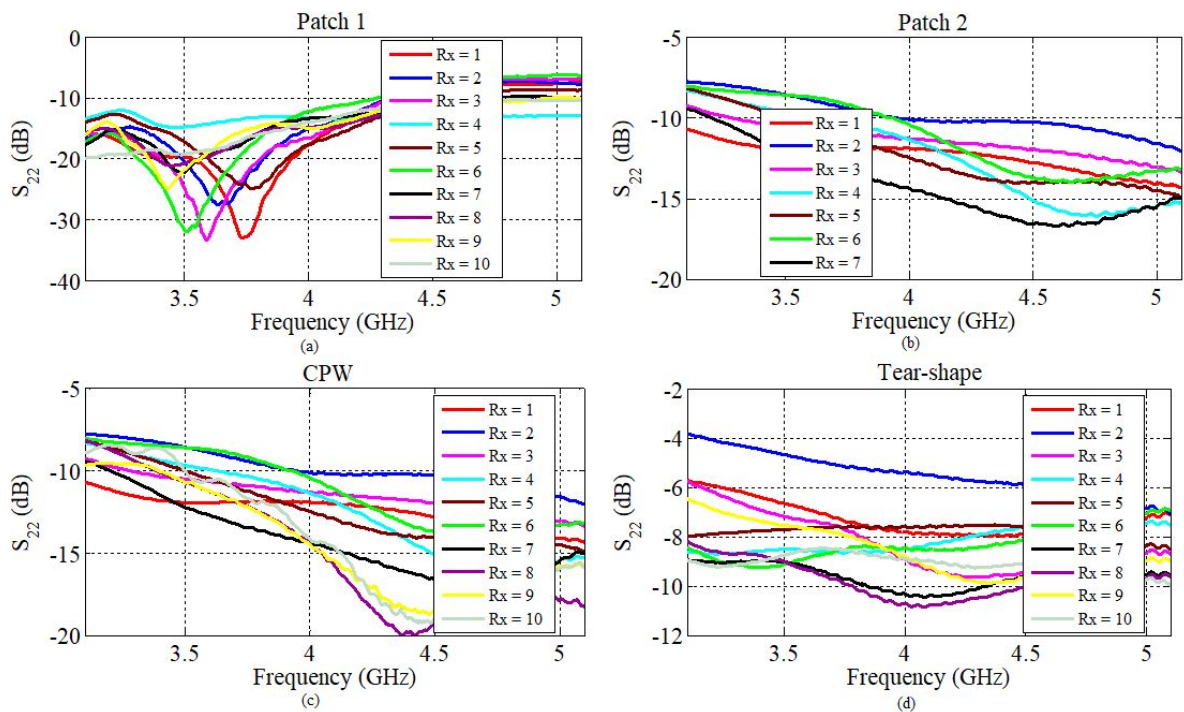

Figure 3.29: $S_{22}$ for the Patch 1 (a), Patch 2 (b), CPW (c) and Tear-shape (d)

\subsection{Offline RF-based Localization}

In this section offline processing of the data collected through simulations and experimental measurements is described. Particularly, the first paragraph describes the two approaches used to estimate the distance between the in-body and on-body antennas (ranging distance): Received Signal Strength (RSS) and Time of Arrival (ToA). Then, the second paragraph details the algorithms used to estimate the coordinates of the in-body antenna in case of one-dimensional, two-dimensional and three-dimensional positioning.

\subsubsection{Ranging distance estimation}

In the following an overview of how the distance between transmitter and receivers (ranging distance) is estimated through RSS and ToA approach is given. 


\subsubsection{Received Signal Strength (RSS)}

As mentioned in Section 2.3, RSS-based localization implies a previous characterization of the propagation channel. Specifically, a path loss model is needed in order to estimate the distance between transmitter and receiver (ranging distance).

Several path loss models for in-body to on-body communications at UWB frequencies have been proposed in literature [2] but none of them is standardized, i.e. none of them is general enough to be adopted as standard for this kind of communications. Therefore, analysis of the propagation channel and path loss models have been derived from the simulations and measurements presented in the previous chapter.

From the measured $S_{21}$ path loss values for each in-body to on-body antenna position were evaluated as follows:

$$
P L_{\text {meas }}(d B)=-10 \log _{10}\left(\overline{|H(f)|^{2}}\right)
$$

where $H(f)$ is the frequency transfer function in $N$ resolution points computed as $H(f)=\left|S_{21}\right| e^{-j \phi S_{21}}$, where $\left|S_{21}\right|$ and $\phi_{21}$ are module and phase in radians of the $S_{21}$, respectively.

Depending on the measurements campaign, path loss values within a minimum and maximum distance between transmitting and receiving antennas were taken into account, in order to ensure that considered measurements are well above the noise level. Then, selected path loss values were fitted by a log-distance approximation model:

$$
P L(d B)=P L_{0, d_{r e f}}(d B)+10 n \log _{10}\left(\frac{d}{d_{r e f}}\right)
$$

where $d$ is the distance between antenna centers, $d_{r e f}$ is the reference distance, $P L_{0, d_{r e f}}$ is the path loss at $d_{r e f}$ and $n$ is the path loss exponent. A reference distance of $1 \mathrm{~cm}$ was considered as it is the most used among the in-body to on-body UWB channel models proposed in literature.

From measurements, the performance of RSS-based positioning were evaluated under two different assumptions:

1. Assuming an ideal receiver capable of detecting all the multipath components of the channel impulse response (ideal case) so through Equation 3.1 the path loss can be precisely estimated as:

$$
P L_{\text {est }}(d B)=P L_{\text {meas }}(d B)
$$

2. Considering a real case scenario, where the receiver usually receives for a given period of time being able to detect only few multipath components 
with power below a certain level from the strongest path. In this case, the path loss can be computed as follows:

$$
P L_{e s t}(d B)=-10 \log _{10} \sum|h(\tau)|_{\text {sel }}^{2}
$$

where $|h(\tau)|_{\text {sel }}=|\operatorname{IFFT}(H(f))|_{\text {sel }}$ are the selected multipath components of the channel impulse response.

From the model proposed in 3.2 an estimation of the ranging distance $d$ can be obtained as follows:

$$
d_{\text {est }}=10^{\frac{P L_{e s t}-P L_{0, d_{r e f}}}{10 n}} \cdot d_{r e f}
$$

where $P L_{e s t}$ is the path loss evaluated using Equation 3.3 or 3.4 if assuming ideal or not ideal channel estimation, respectively.

\subsubsection{Time of Arrival (ToA)}

For ToA-based ranging, as mentioned in Section 2.3, the main issue is the estimation of the propagation velocity of the RF signal travelling across different human tissues.

For each in-body-on-body antenna position, from the measured $S_{21}$ the channel Power Delay Profile (PDP) was calculated as follows:

$$
P D P=10 \log _{10}|h(\tau)|^{2}
$$

where $h(\tau)$ is the Inverse Fast Fourier Transform (IFFT) of the channel matrix $\left(H(f)=\left|S_{21}\right| e^{-j \phi S_{21}}\right)$ in frequency domain. From the PDP the ToA of the direct path signal was evaluated as:

$$
T o A=\tau(\max (P D P))
$$

where $\tau$ is the propagation delays vector.

Then, the ranging distance can be estimated using Equation 2.2.

When considering the homogeneous phantom-based measurements in Section $3.2 .2 .2, \varepsilon_{r}$ in Equation 2.2 is the average relative permittivity $\varepsilon_{r, A v g}$ of the muscle-tissue phantom over the frequency range of interest.

The permittivity of the muscle phantom was measured through a system composed by a VNA, a 1 meter long coaxial cable, a coaxial probe and a computer which controls the VNA and process the measured samples [60]. The output of this system is the dieletric constant, $\varepsilon_{r}^{\prime}$, and the loss factor, $\varepsilon_{r}^{\prime \prime}$, of the relative permittivity of the sample under test:

$$
\varepsilon_{r}=\varepsilon_{r}^{\prime}-j \varepsilon_{r}^{\prime \prime}
$$


From 3.8 the average relative permittivity of the muscle phantom was then evaluated as:

$$
\varepsilon_{r, A v g}=\frac{\sum_{1}^{N} \sqrt{\left|\varepsilon r^{\prime}\right|^{2}+\left|\varepsilon r^{\prime \prime}\right|^{2}}}{N}
$$

where $N$ is the number of resolution points in frequency within the frequency range of interest. Finally, using Equation 2.2 and the calculated values in Equation 3.7 and 3.9, the distance between antennas can be estimated as:

$$
d=V_{p, A v g} \cdot T o A
$$

where $V_{p, A v g}=\frac{c}{\sqrt{\varepsilon_{r, A v g}}}$ is the average propagation velocity.

When considering heterogeneous phantom-based measurements in Section 3.2.2.3, the average propagation velocity $V_{p, A v g}$ was evaluated based on a slightly modified version of the formula proposed in [77], [78]. Particularly, the average velocity across each tissue was weighted by the distance travelled by the signal within each of the tissue with respect to the total travel path length, i.e. the distance between antennas, as follows:

$$
V_{p, A v g}=\frac{d_{\text {est_RSS }}-2}{d_{\text {est_RSS }}} \cdot V_{p, \text { muscle }}+\frac{2}{d_{\text {est_RSS }}} \cdot V_{p, f a t}
$$

where $V_{p, \text { muscle }}=\frac{c}{\sqrt{\varepsilon_{r, \text { muscle }}}}$ and $V_{p, \text { fat }}=\frac{c}{\sqrt{\varepsilon_{r, f a t}}}$ are the average propagation velocity of the signal across the muscle layer and across the fat layer, respectively, $d_{\text {est_RSS }}$ is the distance between antennas in $\mathrm{cm}$, estimated through RSS approach, described in Section 3.3.1.1 and $2 \mathrm{~cm}$ is the thickness of the fat layer (Section 3.2.2.3). $\varepsilon_{r, \text { muscle }}$ and $\varepsilon_{r, \text { fat }}$ were evaluated as in Equation 3.9 over the frequency range of interest.

\subsubsection{Coordinates Estimation}

In this section the algorithms used to estimate the coordinates of the in-body antenna in case of One-Dimensional (1D), Two-Dimensional (2D) and ThreeDimensional (3D) positioning are presented.

\subsubsection{1D Localization}

In order to estimate the ranging distance using the RSS-based technique, a perfect knowledge of the channel was assumed. Therefore, the path loss was precisely computed as in Equation 3.1. In general, this condition is not satisfied but here we wanted to analyze the most ideal case. Through the log-distance fitting model evaluated as in Equation 3.2, an estimation of $d$ can be obtained through Equation 3.5. 
Using the ToA-based approach the ranging distance was estimated through Equation 3.10, being $\varepsilon_{r, \text { Avg }}$ calculated as in Equation 3.9.

The true distance between any on-body antenna and the in-body antenna is given by:

$$
d=\sqrt{\left(x_{I B}-x_{i}\right)^{2}+\left(y_{I B}-y_{i}\right)^{2}+\left(z_{I B}-z_{i}\right)^{2}}
$$

where $\left(x_{I B}, y_{I B}, z_{I B}\right)$ are the coordinates of the in-body antenna and $\left(x_{i}, y_{i}, z_{i}\right)$ the coordinates of the $i$ th receiver with respect to the reference system illustrated in Figure 3.8(a).

Due to the fact that in the measurements campaign described in Section 3.2.2.2.1, only one position of the on-body antenna was considered, only one coordinate of the in-body antenna could be estimated at a time. In fact, from the estimated ranging distance, taking the coordinates system depicted in Figure $3.8(\mathrm{a})$ as reference and by fixing $\mathrm{z}$, the $x_{I B}$ est or $y_{I B}$ est estimated coordinates of the in-body antenna could be calculated using $\overline{3.12}$, depending on the considered direction of movement. This means that by fixing y and considering the in-body antenna movement along $\mathrm{x}$ then $x_{I B_{-} \text {est }}$ could be evaluated, and by fixing $\mathrm{x}$ and considering the in-body antenna movement along $\mathrm{y}$ then $y_{I B \_ \text {est }}$ could be evaluated.

\subsubsection{2D Localization}

Considering the measurement campaigns described in Section 3.2.2.2.2 and 3.2 .2 .3 , only two coordinates of the in-body antenna $\left(y_{I B}, z_{I B}\right)$ could be estimated as all receivers are located in the same $(y-z)$ plane i.e., they share the same $\mathrm{x}$ coordinate.

Ranging distance was estimated through Equation 3.5, assuming ideal and not ideal channel estimation, or through Equation 3.10 considering the RSS or ToA approach, respectively.

The in-body antenna coordinates were estimated through trilateration using a linearized approach [45]. The method is suitable for 3D localization but it could be easily adapted for 2D localization. Such methodology is explained in the following.

Let $C=\left(y_{I B}, z_{I B}\right)$ be the coordinates of the in-body antenna and $R_{i}=$ $\left(y_{i}, z_{i}\right)$ the position of the $i$ th receiver. For $2 \mathrm{D}$ localization, at least three receivers are needed, one taken as reference, to get a linear system in 2 unknowns $y_{I B}, z_{I B}$ in the form of:

$$
A x=b
$$

where $A$ and $x$ are given by 


$$
\begin{gathered}
A=\left(\begin{array}{cc}
y_{1}-y_{n} & z_{1}-z_{n} \\
\vdots & \vdots \\
y_{N}-y_{n} & z_{N}-z_{n}
\end{array}\right) \\
x=\left(\begin{array}{c}
y_{I B}-y_{n} \\
z_{I B}-z_{n}
\end{array}\right)
\end{gathered}
$$

and $b$ is given by

$$
b=\left(\begin{array}{c}
0.5\left(\hat{d}_{n}(C)^{2}+d_{1 n}{ }^{2}-\hat{d}_{1}(C)^{2}\right) \\
\vdots \\
0.5\left(\hat{d}_{n}(C)^{2}+d_{N n}{ }^{2}-\hat{d}_{N}(C)^{2}\right)
\end{array}\right)
$$

where $R_{n}=\left(y_{n}, z_{n}\right)$ is the position of the reference receiver, $\hat{d}_{n}(C)$ is the estimated distance between the in-body antenna and the $n$th reference receiver, $d_{i n}$ is the distance among the $i$ th receiver and the $n$th reference receiver and $\hat{d}_{i}(C)$ is the estimated distance among the in-body antenna and the $i$ th receiver. Finally, $\left(y_{I B}, z_{I B}\right)$ are obtained by solving Equation 3.13 as follows:

$$
x=\left(A^{T} A\right)^{-1} A^{T} b
$$

where $(.)^{T}$ represents the transpose of a matrix. In cases where $\left(A^{T} A\right)$ is nearly singular and poorly conditioned its inverse cannot be calculated. For such cases, the solution can be calculated by back substitution after doing QR decomposition of the matrix A [44].

\subsubsection{3D Localization}

For 3D localization, measurements described in Section 3.2.3 and 3.2.4 were considered.

Ranging distance was estimated using the RSS-based approach, through Equation 3.5, assuming ideal and not ideal channel estimation. ToA approach was not used as results obtained for the $1 \mathrm{D}$ and $2 \mathrm{D}$ case, presented and discussed in the next chapters, showed poor accuracy compared to the RSS-based technique.

The in-body antenna coordinates were estimated through trilateration using a Non Linear Least Square (NLLS) approach [44]. This approach consist in the minimization of the sum of the squares of the errors on the distance:

$$
\min \sum_{i=1}^{N}\left(\sqrt{\left(x_{I B}-x_{i}\right)^{2}+\left(y_{I B}-y_{i}\right)^{2}+\left(z_{I B}-z_{i}\right)^{2}}-d_{i, e s t}\right)^{2}
$$


where $N$ is the number of selected receivers for the localization, $\left(x_{I B}, y_{I B}, z_{I B}\right)$ are the coordinates of the in-body antenna, $\left(x_{i}, y_{i}, z_{i}\right)$ are the coordinates of the $i$ th selected receivers and $d_{i, e s t}$ is the estimated distance between in-body antenna and selected receiver $i$.

The function in 3.18 was minimized through the Levenberg-Marquardt iterative algorithm [49]. This algorithm combines two minimization methods: the gradient descent and the Gauss-Newton method. In the gradient descent method, the sum of the squared errors is reduced by updating the parameters in the steepest-descent direction. In the Gauss-Newton method, the sum of the squared errors is reduced by assuming the least squares function locally quadratic and finding its minimum. The Levenberg-Marquardt method acts more like a gradient-descent method when the parameters are far from their optimal value, and acts more like the Gauss-Newton method when the parameters are close to their optimal value.

An initial starting point is required to start the iterative process. An initial 'guess' for the in-body antenna coordinates can be obtained by solving the system in Equation 3.13 formulated for 3D positioning [45] through the same Linear Least Square (LLS) method described in Section 3.3.2.2. In this dissertation, a fixed starting point was picked, in order to avoid time-consuming solvers and additional complexity when the matrix in Equation 3.13 is close to singularity. $(0,0,0)$ was mostly used as initial point since it was assessed to be far enough from the real solution (true location of the in-body antenna), considering the case-specific reference system. In case this condition was not satisfied, another starting point was chosen according to the same rule.

Considering the data collected during the third in vivo experiment, described in Section 3.2.4.4, an hybrid RSS/AoA scheme was used, besides the RSS-based localization only. For these measurements, in fact, azimuth and elevation angle of the sensors attached to the in-body and on-body antenna were measured along with the distance between antennas and their coordinates. It is important pointing out that in this case the AoA information (azimuth and elevation angle) was provided by the magnetic tracker controlling software but in real applications the AoA needs to be estimated through appropriate algorithms [79].

Figure 3.3.2.1 depicts the measurement setup, highlighting the position of the magnetic tracker and the reference system along with the azimuth $\phi_{i}$ and elevation angle $\alpha_{i}$ between the in-body antenna and the $i$ th on-body antenna.

The azimuth and elevation angle between the in-body and the $i$ th on-body antenna were evaluated as the difference between the azimuth and elevation angle of the sensors, measured by the magnetic tracker, with respect to its reference system. 


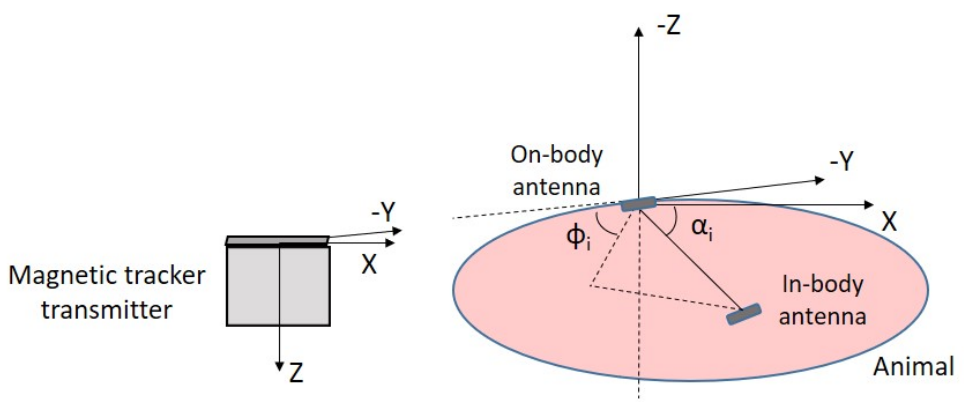

Figure 3.30: Third in vivo measurement setup highlighting azimuth and elevation angles

Azimuth $\phi_{i}$ and elevation $\alpha_{i}$ angle between antennas, considering the reference system in Figure 3.30, can be defined as:

$$
\begin{aligned}
\phi_{i} & =\tan ^{-1} \frac{z_{I B}-z_{i}}{y_{I B}-y_{i}} \\
\alpha_{i} & =\cos ^{-1} \frac{x_{I B}-x_{i}}{d_{i}}
\end{aligned}
$$

where $\left(x_{I B}, y_{I B}, z_{I B}\right)$ are the coordinates of the in-body antenna, $\left(x_{i}, y_{i}, z_{i}\right)$ are the coordinates of the $i$ th receiver and $d_{i}$ is the distance between in-body antenna and receiver $i$ given by $\sqrt{\left(x_{I B}-x_{i}\right)^{2}+\left(y_{I B}-y_{i}\right)^{2}+\left(z_{I B}-z_{i}\right)^{2}}$.

In this case having both, distance and angle, estimations available, the location of the in-body antenna was estimated through the minimization of the sum of the squares of the errors on the distance and on the coordinates [43] considering Equation 3.19 and 3.20. The cost function to be minimized can be defined as in [43]:

$$
\begin{gathered}
\sum_{i=1}^{N}\left\{w_{i}\left(d_{i, e s t}-d_{i}\right)^{2}+\right. \\
w_{N+i}\left(y_{I B} \tan \phi_{i, e s t}-z_{I B}-\left(y_{i} \tan \phi_{i, e s t}-z_{i}\right)\right)^{2}+ \\
\left.w_{2 N+i}\left(x_{I B}-\left(x_{i}+d_{i, \text { est }} \cos \alpha_{i, e s t}\right)\right)^{2}\right\}
\end{gathered}
$$

where $N$ is the number of selected receivers for the localization, $\left(x_{I B}, y_{I B}, z_{I B}\right)$ are the coordinates of the in-body antenna, $\left(x_{i}, y_{i}, z_{i}\right)$ are the coordinates of the $i$ th selected receivers, $\phi_{i, e s t}$ and $\alpha_{i, e s t}$ the azimuth and elevation angle between in-body antenna and $i$ th receiver, derived from those measured by the tracker, $w_{i}$ are weights used to emphasize the contributions of the measurements that 
are more reliable and $d_{i, e s t}$ is the estimated distance between in-body antenna and selected receiver $i$.

The function in 3.21 was minimized through the Levenberg-Marquardt algorithm.

In the absence, as in this case, of a priori knowledge of the standard deviations of the distance, azimuth and elevation angle estimation errors the weights, $w_{i}$, in Equation 3.21 can be simply chosen to be unity. 



\section{Chapter 4}

\section{Localization Results and Discussion}

In this chapter the performance metrics used to evaluate the achieved localization accuracy are firstly introduced. Then, results obtained for onedimensional, two dimensional and three-dimensional positioning through the methodology detailed in Section 3.3 are presented. Furthermore, analysis on the impact of receivers selection as well as of the evaluated path loss models on the localization accuracy is reported. Finally, a section summarizing and discussing the obtained results concludes the chapter.

\subsection{Performance Metrics}

Performance metrics used to assess the goodness of the achieved localization accuracy are detailed in the following. Considering the RSS-based approach, through the log-distance fitting model (Equation 3.2) evaluated in each case (laboratory measurements, simulations, in vivo experiments) an estimation of $d$ can be obtained by solving Equation 3.5. Considering the ToA-based method, through the calculated propagation velocity and ToA, $d$ can be estimated by solving Equation 3.10.

The ranging error and its relative error can then be expressed as:

$$
\begin{gathered}
\text { Ranging Error }=\left|d_{\text {est }}-d\right| \\
\operatorname{Rel}_{\text {RangErr }}=\frac{\text { Ranging Error }}{d}=\frac{\left|d_{\text {est }}-d\right|}{d}
\end{gathered}
$$


where $d$ is the real distance between antennas and $d_{e s t}$ is the estimated one through Equation 3.5.

For the analysis of the localization error tendency, when considering $N_{R X s}$ receivers for positioning, the average ranging error for each considered in-body position can be evaluated as:

$$
A v g_{\text {RangErr }}=\frac{\sum_{i=1}^{N_{R x s}}\left|d_{i, e s t}-d_{i}\right|}{N_{R x s}}
$$

where $d_{i, e s t}$ is the estimated distance between the in-body antenna and the $i$ th receiver obtained through Equation 3.5 and $d_{i}$ is the real distance between the in-body antenna and the $i$ th receiver.

The localization error, LE, for the $3 \mathrm{D}$ case and its relative error can be defined as:

$$
\begin{gathered}
L E=\sqrt{\left(x_{I B}-x_{I B_{-} e s t}\right)^{2}+\left(y_{I B}-y_{I B_{-} e s t}\right)^{2}+\left(z_{I B}-z_{I B_{-} e s t}\right)^{2}} \\
\operatorname{Rel}_{L E}=\frac{L E}{\sqrt{x_{I B}^{2}+y_{I B}^{2}+z_{I B}^{2}}}
\end{gathered}
$$

where $\left(x_{I B}, y_{I B}, z_{I B}\right)$ and $\left(x_{I B \_e s t}, y_{I B \_e s t}, z_{I B}\right.$ est $)$ are the real and estimated coordinates of the in-body antenna, respectively. For the $2 \mathrm{D}$ case, Equations 4.4 and 4.5 are calculated omitting the $\mathrm{x}$-coordinate as for the specific receivers configuration (Figure 3.9 and Figure 3.15) it could not be estimated.

The relative errors on the estimation of the in-body antenna coordinates can be evaluated individually as follows:

$$
\begin{aligned}
& \operatorname{RelEr}_{x_{I B}}=\frac{\left|x_{I B}{ }_{-e s t}-x_{I B}\right|}{\left|x_{I B}\right|} \\
& \operatorname{RelErr}_{y_{I B}}=\frac{\left|y_{I B} e s t-y_{I B}\right|}{\left|y_{I B}\right|} \\
& \operatorname{RelErr}_{z_{I B}}=\frac{\mid z_{I B \_} e s t}{-z_{I B} \mid}
\end{aligned}
$$

where $\left(x_{I B}, y_{I B}, z_{I B}\right)$ and $\left(x_{I B_{-} e s t}, y_{I B_{-} e s t}, z_{I B_{-} e s t}\right)$ are the real and estimated coordinates of the in-body antenna, respectively.

In order to evaluate the error committed in the estimation of the distance "traveled" by the in-body antenna from one location to another, the estimated distance between a set of $3 \mathrm{D}$ positions, $i=1, \ldots, n$ can be defined as in [80] as follows: 
$\widehat{d}=\sqrt{\left(x_{I B_{e s t}, i+1}-x_{I B_{e s t}, i}\right)^{2}+\left(y_{I B_{e s t}, i+1}-y_{I B_{e s t}, i}\right)^{2}+\left(z_{I B_{e s t}, i+1}-z_{I B_{e s t}, i}\right)^{2}}$

where $\left(x_{I B_{e s t}, i+1}, y_{I B_{e s t}, i+1}, z_{I B_{e s t}, i+1}\right)$ and $\left(x_{I B_{e s t}, i}, y_{I B_{e s t}, i}, z_{I B_{e s t}, i}\right)$ are the estimated in-body coordinates for position $i+1$ and position $i$ respectively. Similarly, the real distance between the same set of $3 \mathrm{D}$ positions, $i=1, \ldots, n$ can be expressed by:

$$
d=\sqrt{\left(x_{I B, i+1}-x_{I B, i}\right)^{2}+\left(y_{I B, i+1}-y_{I B, i}\right)^{2}+\left(z_{I B, i+1}-z_{I B, i}\right)^{2}}
$$

where $\left(x_{I B, i+1}, y_{I B, i+1}, z_{I B, i+1}\right)$ and $\left(x_{I B, i}, y_{I B, i}, z_{I B, i}\right)$ are the real in-body coordinates for position $i+1$ and position $i$ respectively.

Finally, the relative and absolute error on the distance "traveled" by the in-body antenna, also referred as tracking error, can be defined:

$$
\begin{aligned}
\text { TrackErr }_{r e l} & =\frac{\widehat{d}-d}{d} \\
\operatorname{TrackEr}_{a b s} & =\widehat{d}-d
\end{aligned}
$$

where $\widehat{d}$ and $d$ are the estimated and real distance calculated through Equation 4.9 and Equation 4.10, respectively.

\subsection{D Positioning}

In this section localization results obtained from the laboratory measurements described in Section 3.2.2.2.1 are reported. It is important reminding that since only one position of the on-body antenna was considered, only one coordinate of the in-body antenna could be estimated at a time, depending on the considered direction of movement on $\mathrm{x}$ or $\mathrm{y}$-axis of the in-body antenna. Results obtained with RSS and ToA approach are described. For the sake of this work, data from 3.1 - $4.1 \mathrm{GHz}$ were considered because for antenna distances larger than $8 \mathrm{~cm}$ measurements beyond $4.1 \mathrm{GHz}$ were below the noise level.

\subsubsection{RSS-based localization}

Path loss values, considering ideal channel estimation and calculated through Equation 3.1, within a distance between antenna centers from $d=2.8 \mathrm{~cm}$ to $d=8 \mathrm{~cm}$ were fitted by a log-distance approximation model (Equation 3.2). Table 4.1 reports the fitting parameters of the log-distance model for a reference 
distance, $d_{r e f}$, of $1 \mathrm{~cm}$, when considering the path loss values related to each $\mathrm{z}$ plane and when considering the path loss values related to all z planes. The path loss exponent, as expected, increases as the misalignment along z-axis between antennas increases being $\mathrm{z}=1.15 \mathrm{~cm}$ and $\mathrm{z}=3.15 \mathrm{~cm}$ the minimum and maximum antennas separation along $\mathrm{z}$.

Table 4.1: Path Loss Model Parameters

\begin{tabular}{l|ccc}
\hline \hline \multirow{2}{*}{$3.1-4.1 \mathrm{GHz}$} & \multicolumn{3}{|c}{ Log-distance model } \\
& $P L_{0, d_{\text {ref }}}$ & $n$ & $\sigma$ \\
\hline $\mathrm{z}=3.15 \mathrm{~cm}$ & -24.0246 & 10.6079 & 4.03 \\
$\mathrm{z}=2.15 \mathrm{~cm}$ & -16.5699 & 10.0283 & 4.31 \\
$\mathrm{z}=1.15 \mathrm{~cm}$ & -0.5845 & 8.5537 & 3.78 \\
\hline all distances & -8.0544 & 9.0429 & 5.13
\end{tabular}

The ranging distance for each in-body to on-body location was then estimated using Equation 3.5, considering the parameters reported in Table 4.1.

The relative errors on the estimation of $x_{I B}, y_{I B}$, calculated as in Equations 4.6 and 4.7 as a function of the in-body antenna actual coordinates, for plane $\mathrm{Z}=1.15 \mathrm{~cm}$ are presented in Figure 4.1, considering the plane related and general (all distances) path loss models reported in Table 4.1.
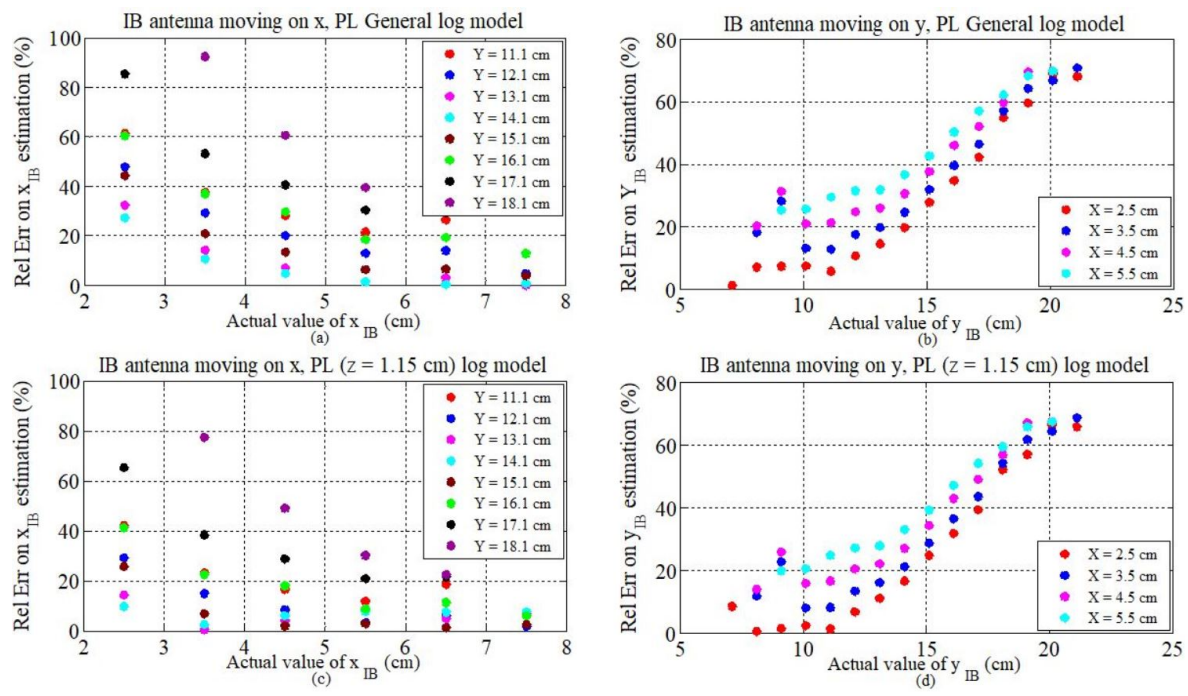

Figure 4.1: Relative errors on the estimation of $x_{I B}$ with (a) general and (c) plane related PL model for $\mathrm{z}=1.15 \mathrm{~cm}$, relative errors on the estimation of $y_{I B}$ with (b) general and $(\mathrm{d})$ plane related $\mathrm{PL}$ model for $\mathrm{z}=1.15 \mathrm{~cm}$ 
Knowing that the position of the on-body antenna is $\left(x_{O B}, y_{O B}, z_{O B}\right)=(0,14.5,0)$ and the initial location of the in-body antenna is $\left(x_{I B}, y_{I B}, z_{I B}\right)=(2.5,3.1,1.15)$ some considerations on the results in Figure 4.1 can be made. When the inbody antenna is moving along $\mathrm{x}$ the lowest error is obtained for $\mathrm{y}=13.1,14.1$, $15.1 \mathrm{~cm}$. In fact, in these locations the two antennas are almost aligned and this results in better localization accuracy. When the in-body antenna is moving along $\mathrm{y}$ the relative error increases with the increment in position along $\mathrm{x}$-axis. Also it can be observed that general and plane related path loss model lead to nearly the same results in terms of localization accuracy. Except for the positions explained above where the error is minimum, overall the relative error, as expected, increases as the distance among the antennas increases. Similar results were obtained for the other two $\mathrm{Z}$ planes.

Figure 4.2 reports the Cumulative Distribution Function (CDF) of the relative error on the estimation of $d$ (distance among antenna centers), calculated as in Equation 4.2, for each z plane, considering the in-body antenna movement along $\mathrm{x}$.

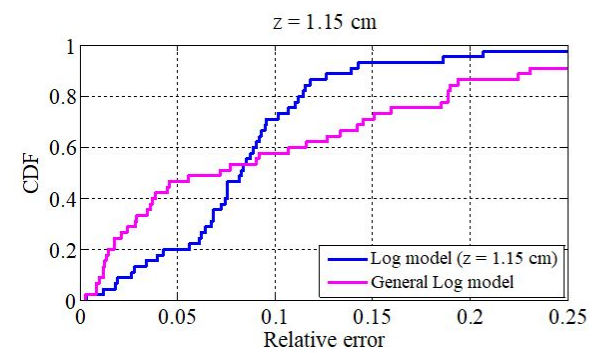

(a)

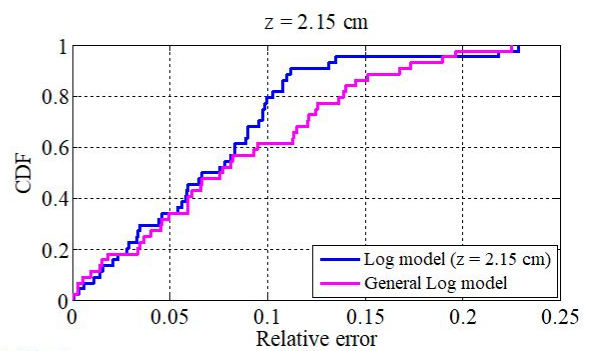

(b)

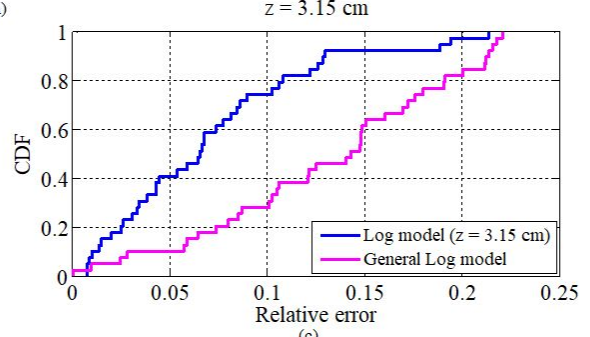

(c)

Figure 4.2: CDF of relative error on the estimation of the ranging distance $d$ for (a) $\mathrm{z}=1.15$ $\mathrm{cm}$, (b) $\mathrm{z}=2.15 \mathrm{~cm}$, (c) $\mathrm{z}=3.15 \mathrm{~cm}$

It can be observed, looking at Figure 4.2, that as the misalignment, i.e. the separation along $\mathrm{z}$, among the antennas increases the plane-related path loss model leads to lower relative error values compared to the general path loss model. As a matter of fact, from $\mathrm{Z}=1.15 \mathrm{~cm}$ to $\mathrm{Z}=3.15 \mathrm{~cm}$ there is an increment of about $5 \%$ in the relative error. This means a $2.5 \%(\sim 0.15-0.2$ 
$\mathrm{cm}$ ) error increase per $\mathrm{cm}$ of misalignment among the antennas. Similar results were obtained for the in-body antenna moving along y.

\subsubsection{ToA-based localization}

For ToA-based localization, ranging distance for each in-body to on-body location was estimated through Equation 3.10, where the average propagation velocity $V_{p, A v g}$, was calculated using as $\varepsilon_{r, A v g}$, the average permittivity of the muscle-tissue phantom over $3.1-4.1 \mathrm{GHz}$ frequency range. Figure 4.3 shows the relative errors, calculated as in Equations 4.6 and 4.7 on the estimation of $x_{I B}(\mathrm{a})$ and $y_{I B}(\mathrm{~b})$, as a function of the in-body antenna actual coordinates, for $\mathrm{z}=1.15 \mathrm{~cm}$.
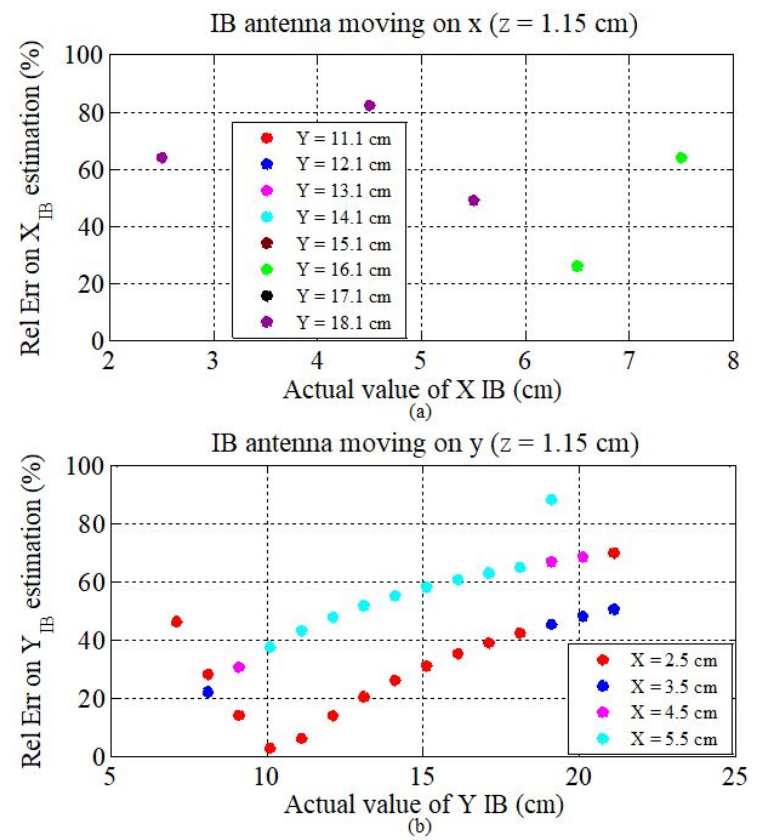

Figure 4.3: Relative errors on the estimation of $x_{I B}$ (a) and $y_{I B}$ (b) for plane $\mathrm{z}=1.15 \mathrm{~cm}$

It can be observed that overall ToA method leads to higher inaccuracy, i.e. higher localization errors, in the position estimation of the in-body antenna. This is due to bandwidth limitations ( $1 \mathrm{GHz}$ in this case) resulting into a poor resolution in the delay domain which causes erroneous detection of the direct path signal. Figure 4.4 shows the Power Delay Profile (PDP) related to a distance among antenna centers equal to $4.02 \mathrm{~cm}$. On the $\mathrm{x}$-axis is the delay 
vector multiplied by the propagation velocity of the RF signal inside the muscle phantom.

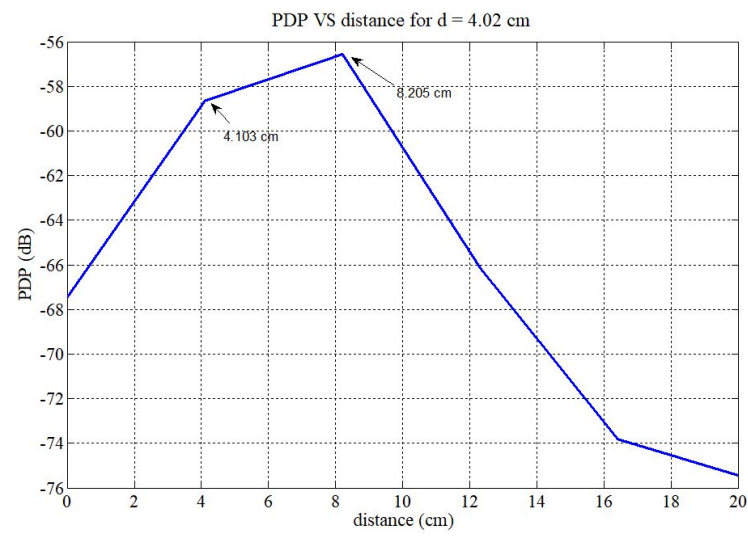

Figure 4.4: Power Delay Profile for distance among antenna centers $d=4.02 \mathrm{~cm}$

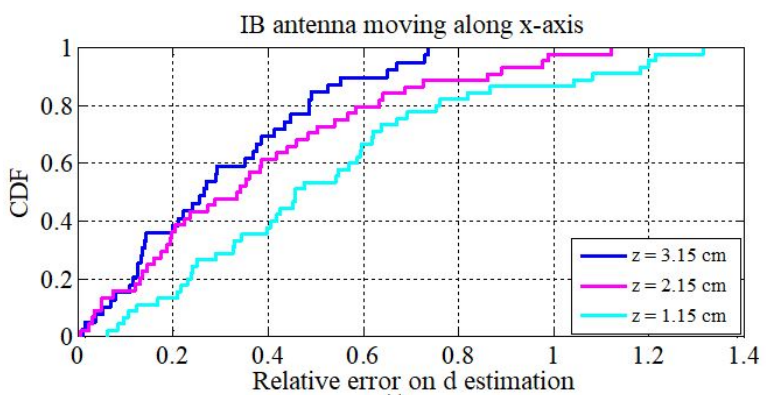

(a)

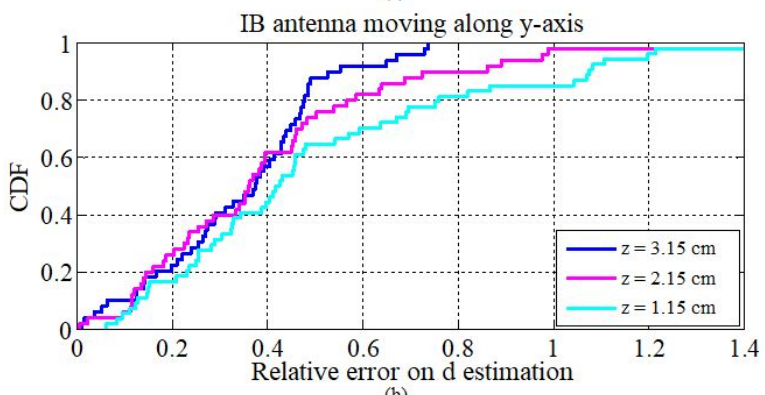

(b)

Figure 4.5: CDF of relative error on the estimation of $d$ for all $\mathrm{z}$ planes, when in-body antenna is moving along $\mathrm{x}$-axis (a) and when is moving along $\mathrm{y}$-axis (b) 
It is worth observing that the resolution between two consecutive points (i.e. delay) is around $4 \mathrm{~cm}$ which implies that two consecutive multipath components arriving with a difference in the path lengths lower than $4 \mathrm{~cm}$ cannot be separately distinguished. Therefore this issue affects the accuracy of the localization. This is more evident in Figure 4.5 which reports the Cumulative Distribution Function (CDF) of the relative error on the estimation of $d$ (distance among antenna centers) for each z plane considered. Due to poor resolution, as shown in Figure 4.4, for distances among the antennas in the range of $4-8 \mathrm{~cm}$ the detected ToA of the direct path signal will generate a distance estimation around $8 \mathrm{~cm}$. This means that when the actual distance among the antennas is near $8 \mathrm{~cm}$ the estimated distance will be closer to this value resulting in, as depicted in Figure 4.5, a reduction of the relative error as the misalignment, i.e. the separation along $\mathrm{z}$, between antennas increases.

\subsection{D Positioning}

In this section localization results obtained from the homogeneous and heterogeneous phantom-based laboratory measurements detailed in Section 3.2.2.2.2 and 3.2.2.3 are reported. It is important reminding that due to the specific configuration of the on-body receivers, only two coordinates of the in-body antenna could be estimated. Results obtained with RSS and ToA approach are presented and discussed.

\subsubsection{RSS-based localization}

\subsubsection{Homogeneous Phantom}

Two-dimensional localization was performed using the homogeneous phantombased setup described in Section 3.2.2.2. From the analysis of the channel transfer function, for both antennas, measurements related to a distance between antennas from $3 \mathrm{~cm}$ to $6.5 \mathrm{~cm}$ and frequency up to $5.1 \mathrm{GHz}$ were selected for the path loss calculation, in order to have the maximum components above the noise level. Path loss values, considering ideal channel estimation and calculated through Equation 3.1, were fitted by a log-distance approximation model (Equation 3.2). Table 4.2 summarizes the path loss models parameters, for a reference distance, $d_{r e f}$, of $1 \mathrm{~cm}$, obtained considering each antenna individually and considering both antennas. The Vivaldi antenna shows a higher path loss exponent $n$, than the other two models, which was expectable from the results [69]. Nevertheless, the variance, $\sigma$, of the omnidirectional patch antenna is surprisingly smaller than the directive Vivaldi's one. As expected, the path loss model resulting from the combination of both antennas has a path loss exponent in between the other two models and a variance higher than the 
other models. This is due to the wide spread between the measured path loss values of the two antennas.

Table 4.2: Path Loss Models for Patch and Vivaldi antenna

\begin{tabular}{c|c|c}
\hline \hline PL Vivaldi & PL Patch & PL both antennas \\
\hline$P L_{0, d_{\text {ref }}}=17.35 \mathrm{~dB}$ & $P L_{0, d_{\text {ref }}}=24.86 \mathrm{~dB}$ & $P L_{0, d_{\text {ref }}}=19.05 \mathrm{~dB}$ \\
$n=7.37$ & $n=5.09$ & $n=6.56$ \\
$\mu \sim 0$ & $\mu \sim 0$ & $\mu \sim 0$ \\
$\sigma=5.72 \mathrm{~dB}$ & $\sigma=4.26 \mathrm{~dB}$ & $\sigma=6.79 \mathrm{~dB}$ \\
\hline \hline
\end{tabular}

The real positions of the in-body antenna, as well as the estimated ones, were evaluated with respect to the magnetic tracker transmitter's reference system (Figure 3.10(b) and Figure 3.9). The ranging distance for each inbody to on-body location was estimated through Equation 3.5, using the parameters reported in Table 4.2. For the estimation of the in-body antenna coordinates, $\left(y_{I B}, z_{I B}\right)$, trilateration using the linearized approach described in Section 3.3.2.2 was employed, considering different combinations of three receivers. Performance were evaluated in terms of relative localization error calculated as in 4.5, omitting the x-coordinate, as for the specific configuration of receivers (Figure 3.9 ) it could not be estimated.

Figure 4.6 shows a comparison of the Cumulative Distribution Function of the relative error for the Patch and Vivaldi antenna, using different combinations of three receivers and the path loss models reported in Table 4.2. Particularly, results for each antenna were evaluated and compared when using the general path loss model, i.e. the model obtained considering both antennas, and the antenna related path loss model. For both antennas, performance obtained using the antenna related path loss models (Figure 4.6(c), (d)) were slightly better than those obtained with the general path loss models (Figure 4.6(a), (b)). The best combination of receivers, leading to lowest error values, is receiver 1, 3 and 5 (taken as reference), i.e. the magenta curve in Figure 4.6. In fact, for this combination of receivers the average ranging error, calculated as in Equation 4.3, was assessed to be the lowest compared to the other receivers combinations. This, in turn, results in better accuracy in the estimation of the in-body antenna coordinates when solving the linearized system of equations in Equation 3.13. Considering the best combination of receivers (i.e 1, 3 and 5 as reference), error values obtained using the Patch antenna and its antenna related model were $2-3 \%$ higher than those obtained using the Vivaldi antenna and its corresponding model. In terms of localization error and considering the same combination of receivers, an average error of $1.6 \mathrm{~cm}$ was obtained with 
the Patch antenna and of $0.98 \mathrm{~cm}$ with the Vivaldi antenna, using the antenna related path loss models.

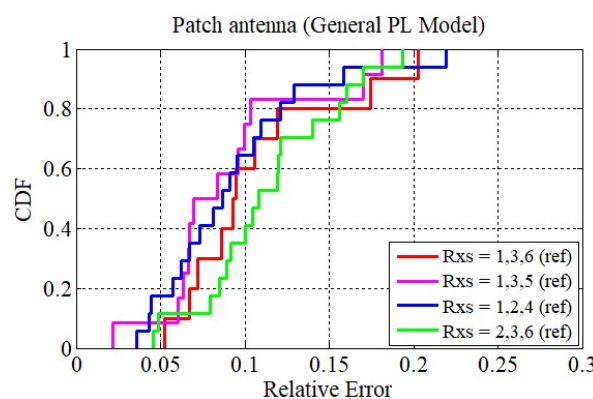

(a)

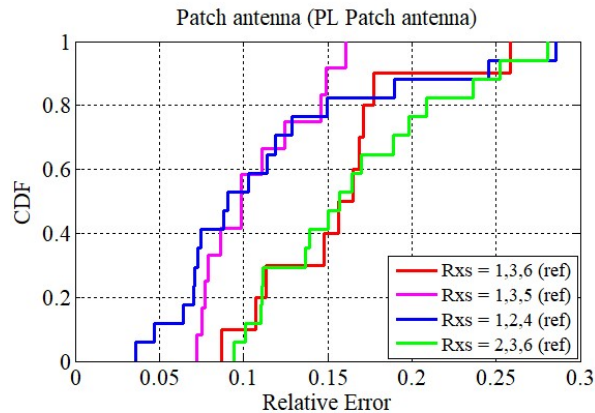

(c)

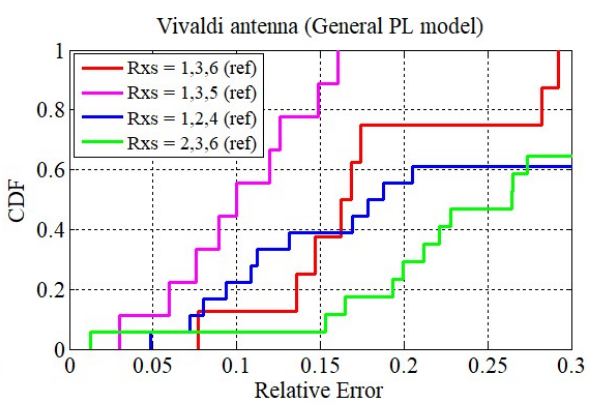

(b)

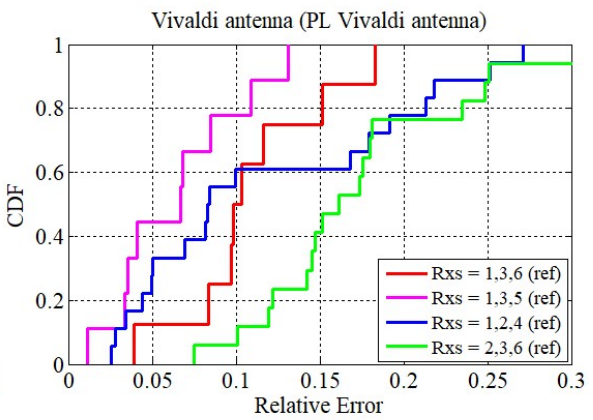

(d)

Figure 4.6: CDF of relative localization error for (a) Patch antenna using the general PL model, (b) for the Patch antenna using the related PL model, (c) for the Vivaldi antenna using the general PL model and (d) for the Vivaldi antenna using the related PL model.

The reason behind this behavior could be explained by the fact that when using the Vivaldi antenna path loss values corresponding to receivers 1,3 and 5 are closer to the antenna related fitting model curve. This turns into a lower average ranging error and, consequently, a slightly lower localization error compared to the one obtained using the Patch antenna.

\subsubsection{Heterogeneous Phantom}

A more realistic two-dimensional localization was performed using the heterogeneous phantom-based setup described in Section 3.2.2.3. Data from 3.1-5.1 $\mathrm{GHz}$ were considered because for antenna distances larger than $8 \mathrm{~cm}$ measurements beyond $5.1 \mathrm{GHz}$ were below the noise level. Path loss values evaluated as in Equation 3.1 were fitted through Equation 3.2 , being $d_{r e f}=1 \mathrm{~cm}, P L_{0, d_{\text {ref }}}$ $=-24.43 \mathrm{~dB}$ and $n=9.69$. In order to estimate the $\left(y_{I B}, z_{I B}\right)$ coordinates 
of the in-body antenna different combination of three receivers (one taken as reference) were considered to directly solve the linearized system in Equation 3.13 with two equations. Figure 4.7(a) depicts the Cumulative Distribution Function (CDF) of the relative localization error, computed as in Equation 4.5 , omitting the $\mathrm{x}$-coordinate, for three different combinations of three receivers. In Figure 4.7(b) the true locations of the in-body antenna (given by the magnetic tracker) are represented versus the estimated ones for the same combinations of receivers. Results show how the receivers selected for localization impact the accuracy of the results. Particularly, the combination of receivers 2,4 and 3 , taken as reference, leads to lower relative error values compared to the other combinations, as it is experiencing on average the highest level of received power [81], [82]. Similar results were obtained for the same combinations of receivers, when considering the same measurements but in a narrower frequency band (3.1-4.1 GHz) [83].

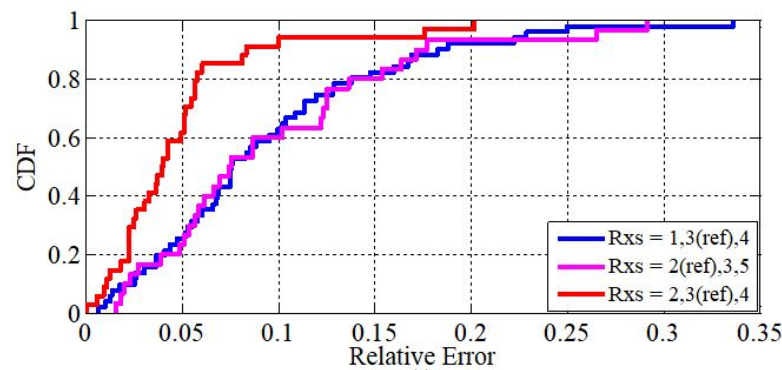

(a)

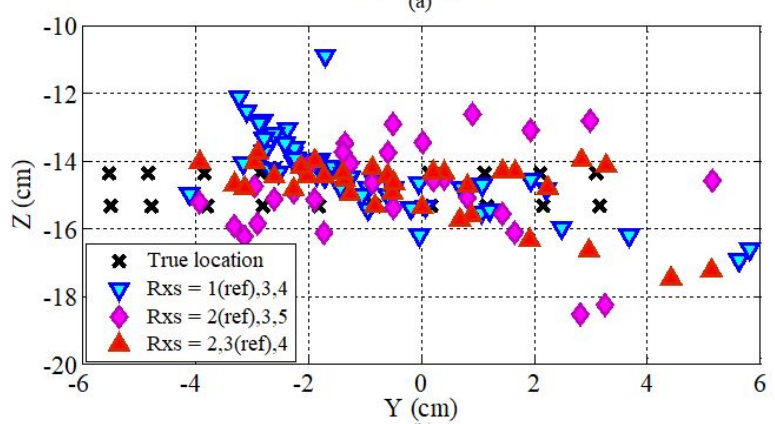

(b)

Figure 4.7: CDF of relative localization error (a), and true vs estimated location of in-body antenna (b) for 2D RSS-based localization

Considering the combination of receivers leading to the best performance, i.e. receivers 2, 4 and 3 as reference (Figure 4.7), localization accuracy was evaluated and compared in case of ideal and not ideal channel estimation assumption, detailed in Section 3.3.1.1. As a reminder, in case of ideal channel 
estimation, the path loss was computed as in Equation 3.1. In case of not ideal channel estimation, the path loss was calculated through Equation 3.4, by selecting all the multipath components whose power is above or equal to the maximum of the Power Delay Profile minus a certain threshold, specifically $5 \mathrm{~dB}, 10 \mathrm{~dB}, 20 \mathrm{~dB}$, respectively. Figure 4.8 (a) shows that considering the components with power below $10 / 20 \mathrm{~dB}$ from the maximum (magenta and green curve), almost same performance as for the ideal channel estimation are obtained, while slightly worse performance is observed when considering the components below $5 \mathrm{~dB}$ (blue curve). This is also noticeable in Figure 4.8(b) where, using the same combination of receivers, an example of tracking considering the in-body antenna moving in steps of $1 \mathrm{~cm}$ along $\mathrm{y}$-axis, for $\mathrm{x}=1$ and $\mathrm{z}=2$, is presented.
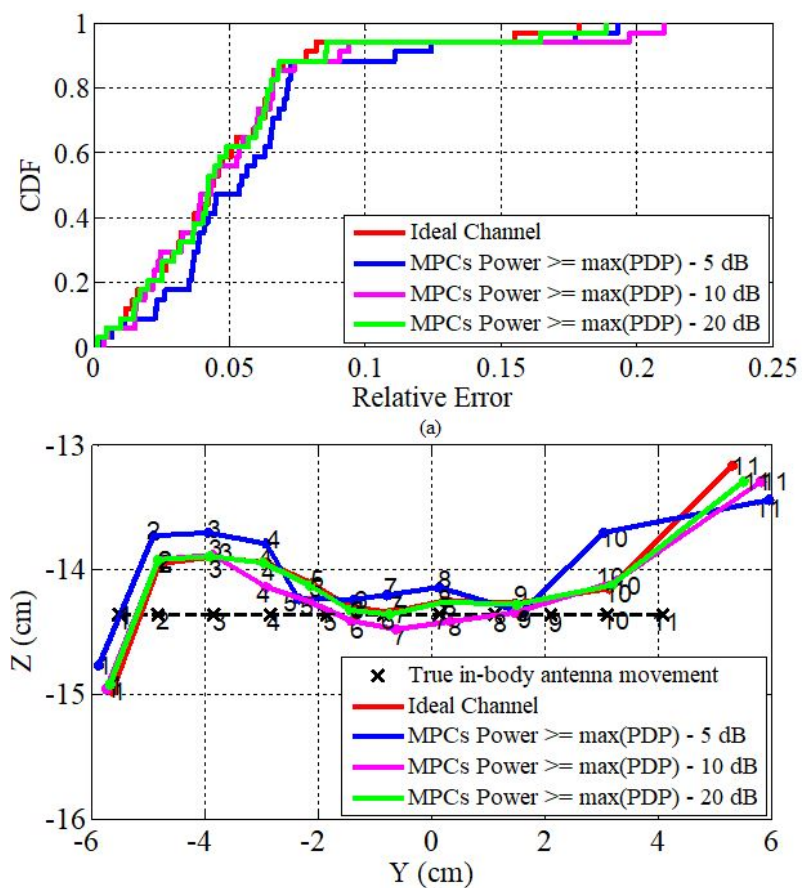

(b)

Figure 4.8: CDF of relative localization error (a) and example of tracking for in-body antenna moving along $\mathrm{y}$-axis, for $\mathrm{x}=1$ and $\mathrm{z}=2(\mathrm{~b})$, for ideal and not ideal channel estimation

It is important to mention that for this track, the distance between the inbody antenna and some on-body receivers was sometimes higher than $8 \mathrm{~cm}$. Thus, for such points the path loss model used for ranging estimation does not include such distances, leading to some inaccuracies. In fact, it is worth 
observing in Figure 4.8(b) that for all the considered cases, the estimation error is higher for the outer points of movement of the in-body antenna i.e., 1-4, 10, 11. For these positions the distance between in-body antenna and receivers 2, 3 (see Figure 3.15) is outside the region of validity of the evaluated path loss model. This means more inaccuracy in the ranging distance calculation in Equation 3.5 and consequently more uncertainty in the estimation of the inbody antenna coordinates $\left(y_{I B}, z_{I B}\right)$. Regarding the localization performance, considering ideal channel estimation an average relative localization error of $4.7 \%$ corresponding to a Localization Error (LE) of $0.72 \mathrm{~cm}$ was achieved. For not ideal channel estimation, considering components with power below 5 $\mathrm{dB}$ from the maximum, an average relative error of $5.7 \%(\mathrm{LE}=0.86 \mathrm{~cm})$ was obtained. This means that in a realistic scenario (not ideal channel estimation) the inability of the receiver to perfectly characterize the channel affects the positioning accuracy leading to an increase of $1 \%$ in the localization error, in this case under study.

\subsubsection{ToA-based localization}

In order to test the ToA approach in a more realistic scenario, compared to the homogeneous phantom case, 2D localization was performed using the heterogeneous phantom-based measurements detailed in Section 3.2.2.3. Ranging distance for each in-body to on-body position, for antenna distances within 8 $\mathrm{cm}$, was estimated through Equation 3.10 where the average propagation velocity $V_{p, A v g}$ was calculated using Equation 3.11, by evaluating through Equation 3.9 the average permittivity of the muscle and fat layer over $3.1-5.1 \mathrm{GHz}$ frequency range. Figure 4.9 shows in (a) the $\mathrm{CDF}$ of the relative localization error calculated as in Equation 4.5, omitting the x-coordinate, and in (b) the true versus estimated location of the in-body antenna, for the same three different combinations of receivers considered for the RSS-based approach in Section 4.3.1.2.

It can be observed, in Figure 4.9(a), that the relative error significantly increases compared to the RSS approach (Figure 4.7). Considering the best combination of receveirs for the RSS-based method, i.e. receivers 2,4 and 3, taken as reference, an average absolute localization error (Equation 4.4) of 7.73 $\mathrm{cm}$ was obtained for the same combination and ToA-based approach.

Looking at Figure 4.9(b) it is worth observing that all the estimated location, $\left(y_{I B}, z_{I B}\right)$, of the in-body antenna are condensed more or less in the same area. The first cause of this behavior is the limited resolution in the delay domain as for the homogeneous phantom case (Section 4.2.2). In fact, looking at an example of the power delay profile, shown in Figure 4.10 for a distance between antenna centers of $7.54 \mathrm{~cm}$, the resolution between two consecutive points (i.e. delays) is more than $4 \mathrm{~cm}$, which implies that two consecutive 
multipath components arriving with a difference in the path lengths lower than $4.7 \mathrm{~cm}$ cannot be separately distinguished.
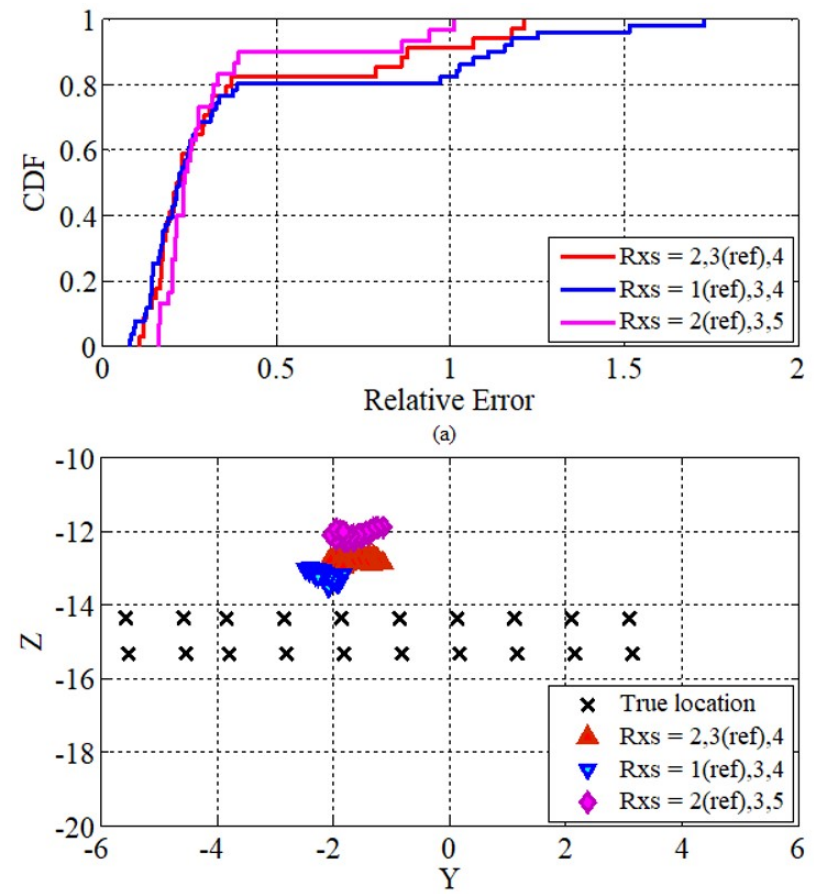

(b)

Figure 4.9: CDF of relative localization error (a) and true vs estimated location of in-body antenna (b), for 2D ToA-based localization

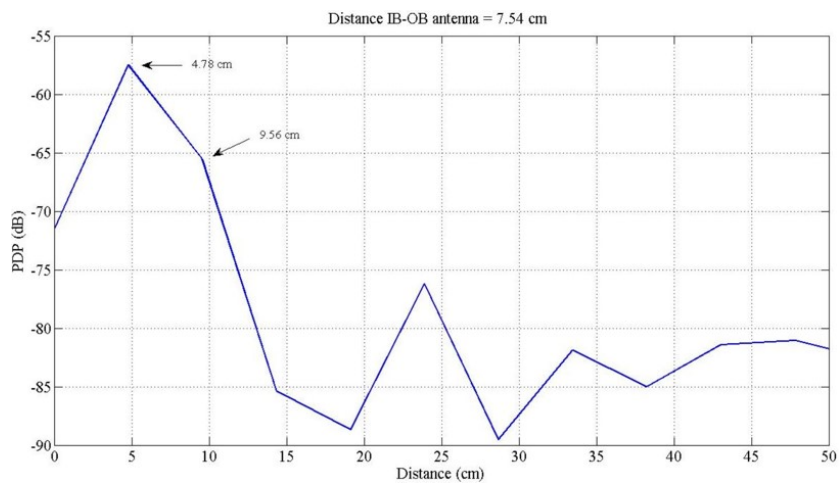

Figure 4.10: Power Delay Profile for distance between antennas of $7.54 \mathrm{~cm}$ 
The second factor affecting the localization accuracy, is that the propagation velocity of the RF signal is not constant over the different considered human tissues. This implies the use of a model to approximate it (in this case Equation 3.11 ) which could be source of possible inaccuracies.

\subsection{D Positioning}

This section reports the three-dimensional positioning results obtained from the data collected through the simulations and in vivo experiments described in Section 3.2.3 and 3.2.4, respectively. Compared to the laboratory campaigns, in this case more tissues were involved in the measurements. Since ToA approach presented resolution issues for the frequency band of interest $(3.1-5.1 \mathrm{GHz})$ and showed poor accuracy when considering one (homogeneous phantom) and two (heterogeneous phantom) human tissues, 3D localization was performed using the RSS-based approach only.

\subsubsection{RSS-based localization}

\subsubsection{Simulations}

From the simulations setup presented in Figure 3.16, which considers five onbody antenna locations and four in-body positions, path loss parameters of the log-distance fitting model (Equation 3.2) $P L_{0, d_{r e f}}=-15.83$ and $n=10.52$ were obtained, considering a reference distance, $d_{r e f}$, of $1 \mathrm{~cm}$.

In order to estimate the $\left(x_{I B}, y_{I B}, z_{I B}\right)$ coordinates of the in-body antenna, the Non Linear Least Square method described in Section 3.3.2.3 was applied using different combinations of four receivers. Figure 4.11 shows the relative localization error, evaluated as in Equation 4.5, obtained for the four simulated in-body positions (located at 5, 6, 6.3 and $7 \mathrm{~cm}$ with respect to the origin of the reference system (Figure 3.16) for five different combinations of four receivers. Results shows that for the first three in-body positions the lowest error is obtained with the combination of receivers $1,2,3,5$. These receivers for all four in-body locations, are experiencing the lowest path loss, i.e. the highest received power. This can be observed in the histogram in Figure 4.12 which depicts the average path loss experienced by each combination of four receivers, represented by a different colorbar, for the four considered in-body locations. For the combination 1, 2, 3, 5, the orange bar, the average experienced path loss is the lowest compared to the other combination of receivers. Therefore, it is reasonable to expect higher positioning accuracy, i.e. lower localization errors, using these four receivers rather than other combinations. However, this is true, only for three in-body positions. For the in-body location at 7 $\mathrm{cm}$, as shown in Figure 4.11, the lowest error is achieved by the combination 
of receivers $1,2,3,4$ which on average experiences the lowest average ranging error, calculated as in Equation 4.3, compared to the other combinations of receivers. A more detailed explanation related to this behavior is discussed in Section 4.5.

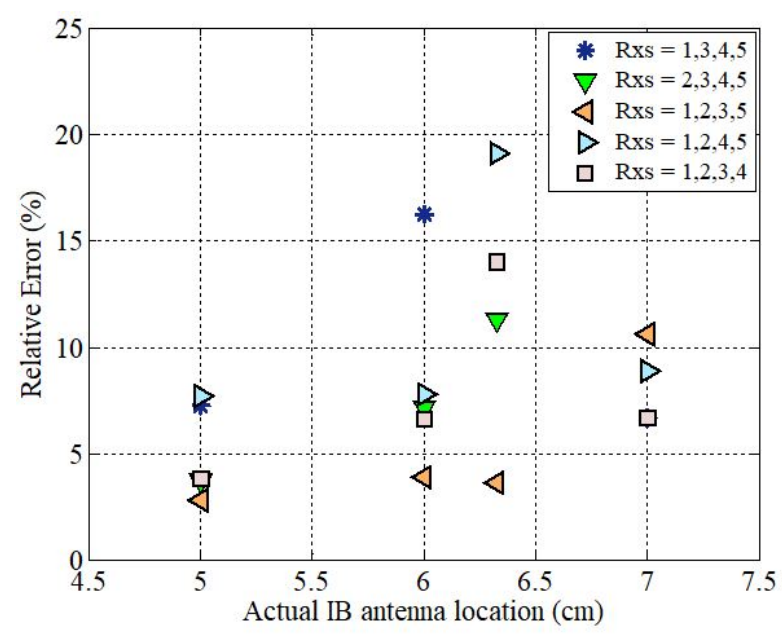

Figure 4.11: Relative localization error vs actual in-body antenna location for different combinations of four receivers

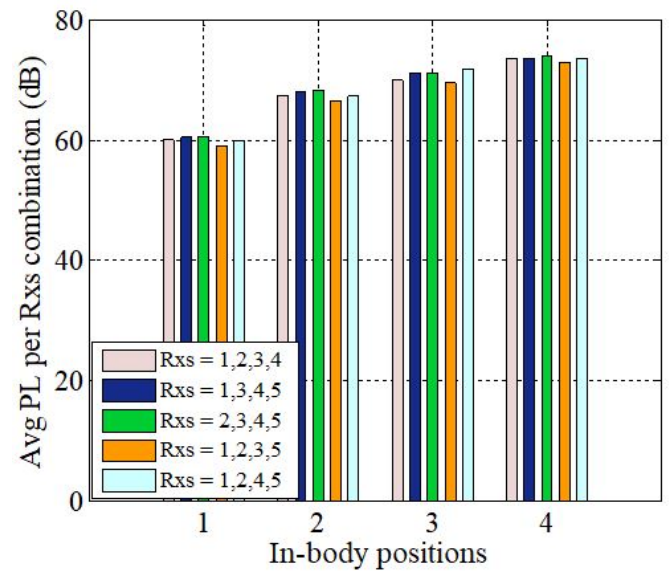

Figure 4.12: Histogram of average path loss per in-body position for different combinations of receivers 
Considering the best combination of receivers (the one leading to the lowest error) for each in-body location, through Equation 4.4, a localization error of $0.14 \mathrm{~cm}, 0.23 \mathrm{~cm}, 0.23 \mathrm{~cm}$ and $0.45 \mathrm{~cm}$ was obtained for in-body position at 5 $\mathrm{cm}$, at $6 \mathrm{~cm}$, at $6.3 \mathrm{~cm}$ and at $7 \mathrm{~cm}$, respectively.

From the supplementary simulations conducted using the same setup and presented in Figure 3.17, path loss parameters $P L_{0, d_{r e f}}=-10.34$ and $n=$ 9.79 were obtained. Localization accuracy was evaluated and compared in case of ideal and not ideal channel estimation assumption (Section 3.3.1.1), as performed for the $2 \mathrm{D}$ case, considering the heterogeneous phantom-based measurements. In case of not ideal channel estimation, the path loss was calculated through Equation 3.4, by selecting the multipath components whose power is above or equal to the maximum of the power delay profile minus $5 \mathrm{~dB}, 10 \mathrm{~dB}$ and $20 \mathrm{~dB}$. Performance evaluated considering components with power below $10 / 20 \mathrm{~dB}$ from the maximum resulted to be very close to those obtained for ideal channel estimation (Equation 3.1). Therefore, Figure 4.13 reports the relative localization error evaluated considering ideal channel estimation in (a) and considering the multipath components with power below $5 \mathrm{~dB}$ from the maximum in (b), using the receivers experiencing the highest level of received power, firstly starting with four and then increasing the number up to nine. It can be observed that for in-body position at $4 \mathrm{~cm}, 5 \mathrm{~cm}$ and $6 \mathrm{~cm}$ the localization error is slightly lower when using not ideal channel estimation. The opposite trend is observed for in-body at $6.3 \mathrm{~cm}, 7 \mathrm{~cm}, 7.2 \mathrm{~cm}$ and $8 \mathrm{~cm}$.

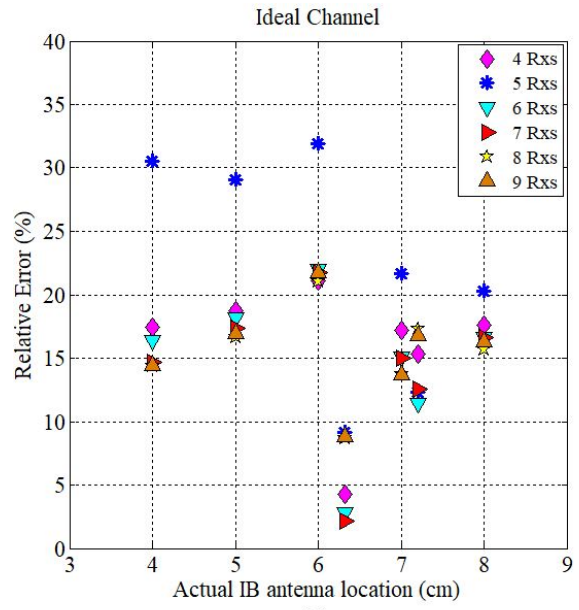

(a)

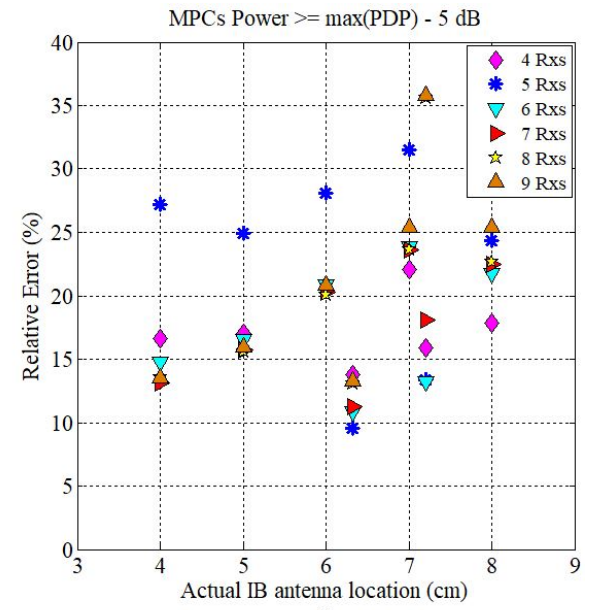

(b)

Figure 4.13: Relative localization error for ideal channel estimation (a) and for not ideal channel estimation (b), considering different number of receivers 
This behavior can be explained looking at the distribution of the path loss values (an example is reported in Figure 4.14) corresponding to the receivers selected for the localization with respect to the logarithmic fitting model. Figure 4.14 shows the selected path loss values (pink triangles), related to the five receivers experiencing the highest level of received power for in-body position at $5 \mathrm{~cm}$, using ideal (a) an not ideal (b) channel estimation, considering the multipath components with power below $5 \mathrm{~dB}$ from the maximum of the power delay profile. For both cases, the same combination of five receivers $(1,2,5,6,7)$ was the one experiencing the highest level of received power.

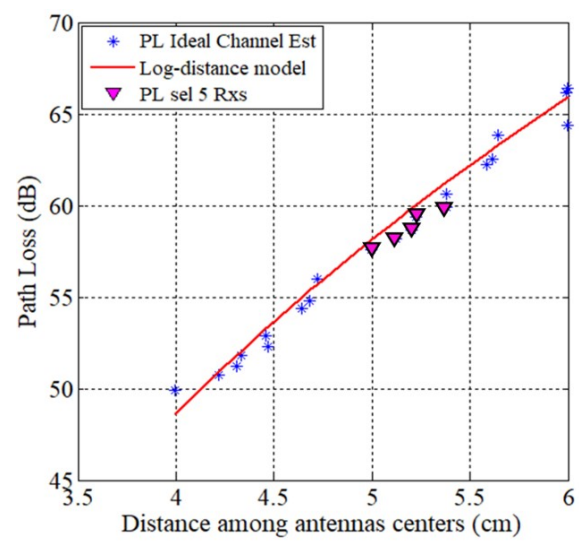

(a)

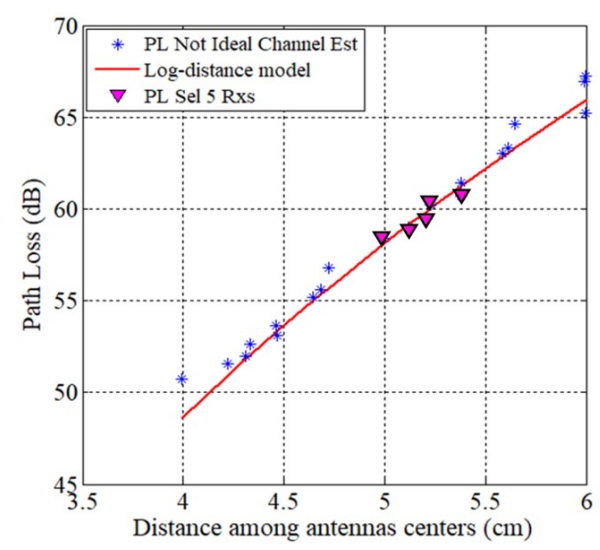

(b)

Figure 4.14: Simulated path loss values and fitting model along with path loss values of selected receivers for in-body position at $5 \mathrm{~cm}$, considering ideal (a) and not ideal (b) channel estimation

It can be observed in Figure 4.14 that, for both cases, ideal and not ideal channel estimation, the distribution of the estimated ranging distances, through the selected path loss values, around the distance to estimate (i.e. $5 \mathrm{~cm}$ ) is very similar. However, for not ideal channel estimation (4.14(b)), the average ranging error, calculated as in Equation 4.3, was slightly lower $(0.05 \mathrm{~cm})$ than the one obtained for the ideal case $(0.1 \mathrm{~cm})$, as the selected path loss values are closer to the fitting model curve. Therefore, when applying the minimization algorithm (3.18), a better estimation of the in-body antenna coordinates can be achieved for the not ideal case. Same explanation holds for in-body position at $4 \mathrm{~cm}$ and at $6 \mathrm{~cm}$. For the remaining in-body positions (at $6.3 \mathrm{~cm}, 7 \mathrm{~cm}, 7.2$ $\mathrm{cm}$ and $8 \mathrm{~cm}$ ) ideal channel estimation resulted in lower localization errors for the same behavior, i.e. selected path loss values lead to lower ranging errors with respect to the not ideal case. 
Considering the best combination of receivers for each in-body position, Table 4.3 reports the localization errors in $\mathrm{cm}$ obtained for ideal and not ideal channel estimation.

Table 4.3: Localization error for ideal and not ideal channel estimation

\begin{tabular}{c|c|c}
\hline \hline IB Pos $(\mathrm{cm})$ & $\begin{array}{c}\text { Ideal } \\
\text { LE }(\mathrm{cm})\end{array}$ & $\begin{array}{c}\text { Not Ideal } \\
\text { LE }(\mathrm{cm})\end{array}$ \\
\hline 4 & 0.58 & 0.52 \\
5 & 0.85 & 0.80 \\
6 & 1.26 & 1.20 \\
6.3 & 0.13 & 0.60 \\
7 & 0.96 & 1.54 \\
7.2 & 0.82 & 0.94 \\
8 & 1.25 & 1.43 \\
\hline \hline
\end{tabular}

\subsubsection{In Vivo}

\subsection{First Experiment}

From the first in vivo experiment described in Section 3.2.4.2, data from 3.1-5.1 GHz were considered because for antenna distances larger than 8-9 cm measurements beyond $5.1 \mathrm{GHz}$ were below the noise level [70]. Path loss values related to antennas distances below or equal to $8 \mathrm{~cm}$ were fitted through Equation 3.2 , resulting $P L_{0, d_{\text {ref }}}=21.84 \mathrm{~dB}$ and $n=5.44$, considering a reference distance of $d_{\text {ref }}=1 \mathrm{~cm}$.

In order to estimate the $\left(x_{I B}, y_{I B}, z_{I B}\right)$ coordinates of the in-body antenna, the Non Linear Least Square method described in Section 3.3.2.3 was applied using different combinations of receivers. Table 4.4 shows the relative and absolute localization error, evaluated as in Equation 4.5 and 4.4 respectively, obtained for the two measured in-body positions, named $I B 1$ and $I B 2$, by selecting the receivers experiencing the highest level of received power, starting with four up to thirteen.

From the results reported in Table 4.4, it can be observed that, by increasing the number of receivers from 4 up to 13, the relative localization error slightly decreases for in-body position IB1 but not for position IB2. In fact, passing from 10 to 13 receivers means, for in-body position IB1 having one receiver outside the region of validity of the path loss model $(>8 \mathrm{~cm})$ and for position IB2 having three of them. This affects the ranging accuracy and adds uncertainty when applying the minimization error algorithm. As for both inbody positions fairly good localization errors were achieved using 10 receivers, 
performance considering ideal and not ideal channel estimation were evaluated applying the Levenberg-Marquardt algorithm by selecting those ten receivers experiencing the highest power.

Table 4.5 and Table 4.6 report, for in-body position IB1 and IB2, respectively, the localization error and its relative error, as well as the relative and absolute errors on the estimation of $\left(x_{I B}, y_{I B}, z_{I B}\right)$, for ideal and not ideal channel estimation case, considering the multipath components with power below $10 \mathrm{~dB}$ and $5 \mathrm{~dB}$ from the maximum of the power delay profile.

Table 4.4: Localization error for different number of receivers

\begin{tabular}{c|cc|cc}
\hline \hline \multirow{2}{*}{$\mathrm{N}$ Rxs } & \multicolumn{2}{|c|}{$I B 1$} & \multicolumn{2}{c}{$I B 2$} \\
& $\operatorname{Rel}_{L E}(\%)$ & $\mathrm{LE}(\mathrm{cm})$ & $\operatorname{Re}_{L E}(\%)$ & $\mathrm{LE}(\mathrm{cm})$ \\
\hline 4 & 1.91 & 1.24 & 4.61 & 3.01 \\
7 & 1.81 & 1.19 & 1.41 & 0.93 \\
10 & 1.48 & 0.97 & 1.40 & 0.91 \\
13 & 1.21 & 0.79 & 2.82 & 1.85 \\
\hline \hline
\end{tabular}

Table 4.5: Localization errors for in-body position IB1

\begin{tabular}{c|c|c|c}
\hline \hline & Ideal & $-10 \mathrm{~dB}$ & $-5 \mathrm{~dB}$ \\
\hline $\operatorname{Rel}_{L E}(\%)$ & 1.48 & 1.27 & 1.11 \\
$\operatorname{LE}(\mathrm{cm})$ & 0.97 & 0.83 & 0.72 \\
$\operatorname{RelErr}_{x_{I B}}(\%)$ & 1.03 & 0.93 & 0.81 \\
$\operatorname{AbsErr}_{x_{I B}}(\mathrm{~cm})$ & 0.66 & 0.60 & 0.52 \\
$\operatorname{RelErr}_{y_{I B}}(\%)$ & 5.93 & 4.85 & 2.87 \\
$\operatorname{AbsErr}_{y_{I B}}(\mathrm{~cm})$ & 0.28 & 0.23 & 0.14 \\
$\operatorname{RelErr}_{z_{I B}}(\%)$ & 6.40 & 5.19 & 4.77 \\
$\operatorname{AbsErr}_{z_{I B}}(\mathrm{~cm})$ & 0.65 & 0.52 & 0.48 \\
\hline \hline
\end{tabular}

Table 4.6: Localization errors for in-body position IB2

\begin{tabular}{c|c|c|c}
\hline \hline & Ideal & $-10 \mathrm{~dB}$ & $-5 \mathrm{~dB}$ \\
\hline $\operatorname{Rel}_{L E}(\%)$ & 1.40 & 1.71 & 1.86 \\
$\operatorname{LE}(\mathrm{cm})$ & 0.91 & 1.12 & 1.21 \\
$\operatorname{RelErr}_{x_{I B}}(\%)$ & 0.55 & 0.78 & 0.41 \\
$\operatorname{AbsErr}_{x_{I B}}(\mathrm{~cm})$ & 0.35 & 0.50 & 0.26 \\
$\operatorname{RelErr}_{y_{I B}}(\%)$ & 6.44 & 8.65 & 5.53 \\
$\operatorname{AbsErr}_{y_{I B}}(\mathrm{~cm})$ & 0.21 & 0.29 & 0.18 \\
$\operatorname{RelErr}_{z_{I B}}(\%)$ & 8.34 & 9.81 & 11.99 \\
$\operatorname{AbsErr}_{z_{I B}}(\mathrm{~cm})$ & 0.82 & 0.96 & 1.17 \\
\hline \hline
\end{tabular}


Results, considering ideal and not ideal channel, show that the lowest localization errors were obtained in the estimation of $x_{I B}$ for both in-body positions. As reported in Table 4.5, for in-body position IB1 error values calculated considering ideal channel are slightly higher than those obtained for the not ideal channel case. For in-body position IB2 same behavior can be observed in the estimation of $x_{I B}$ and $y_{I B}$, in Table 4.6. Although the difference between error values in both cases is not critical, this behavior closely depends on the evaluated path loss model and on the distribution of the selected path loss values, used for ranging estimation.

Figure 4.15 shows the distribution of the path loss values corresponding to the ten receivers selected for localization of in-body IB1 with respect to the logarithmic fitting model, for the ideal (a) and not ideal (b) channel estimation case.

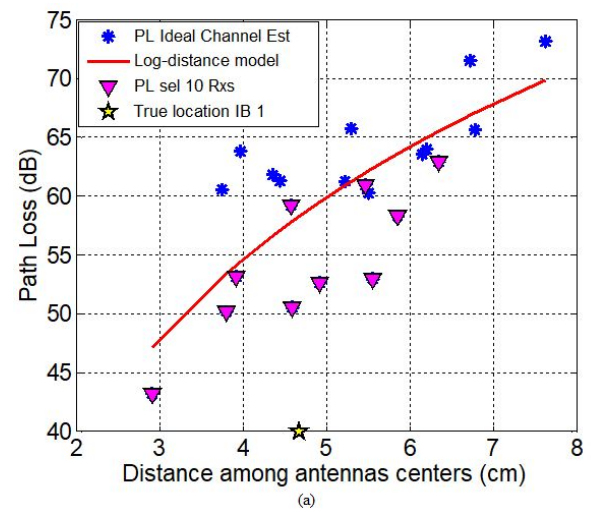

(a)

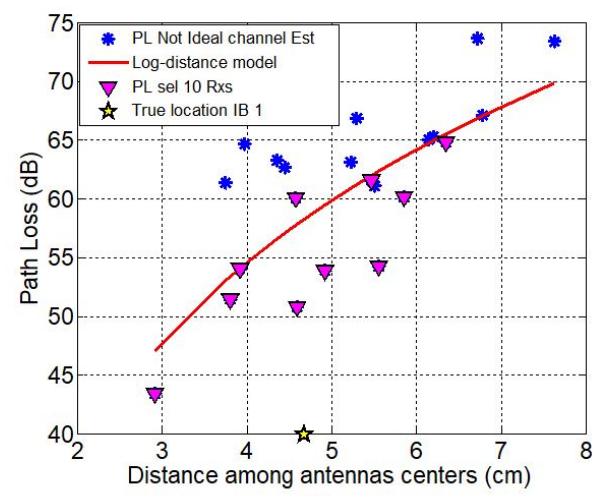

(b)

Figure 4.15: Measured path loss values and fitting model along with path loss values of selected receivers for in-body position IB1, considering ideal (a) and not ideal (b) channel estimation

Not ideal channel estimation refers to path loss values calculated considering the multipath components with power below $5 \mathrm{~dB}$ from the maximum of the PDP. The yellow star indicates the true location of in-body position IB1 with respect to the central receiver Rx5 (Figure 3.19(b)). The distribution of the selected path loss values (magenta triangles) around the distance to estimate (yellow star), considering ideal and not ideal channel estimation, is very similar. However, as observed for the simulations case (4.4.1.1), the average ranging error, calculated as in Equation 4.3 , is slightly lower $(0.61 \mathrm{~cm})$ for not ideal estimation compared to the ideal one $(0.77 \mathrm{~cm})$ as some of the selected path loss values are closer to the fitting model curve. Therefore, being the estimates of the ranging distances well distributed around the distance to estimate (yellow 
star) and more accurate with respect to the ideal case, this can explain the error decrease when considering not ideal channel estimation.

\subsection{Second Experiment}

Considering the second in vivo experiment described in Section 3.2.4.3, for the sake of comparison with the results obtained from the first in vivo as well as from simulations, data from $3.1-5.1 \mathrm{GHz}$ were considered. Performance of RSS-based localization were evaluated and compared for the three different employed on-body antennas to assess whether one of the three is better than the others.

Path loss models were evaluated for antenna distances up to $11 \mathrm{~cm}$ through Equation 3.2, for each on-body antenna as well as considering the measured path loss by all the three on-body antennas. Table 4.7 reports the derived antenna related and general path loss model, for a reference distance, $d_{r e f}$, of $1 \mathrm{~cm}$.

Table 4.7: Path Loss Models for Patch 1, Patch 2, Directive antenna and for all antennas

\begin{tabular}{c|c|c|c}
\hline \hline PL Patch 1 & PL Patch 2 & PL Directive & PL all antennas \\
\hline$P L_{0, d_{\text {ref }}=3.09 \mathrm{~dB}}$ & $P L_{0, d_{\text {ref }}=2.06 \mathrm{~dB}}$ & $P L_{0, d_{\text {ref }}}=11.20 \mathrm{~dB}$ & $P L_{0, d_{r e f}}=5.30 \mathrm{~dB}$ \\
$n=7.61$ & $n=8.03$ & $n=7.20$ & $n=7.63$ \\
$\mu \sim 0$ & $\mu \sim 0$ & $\mu \sim 0$ & $\mu \sim 0$ \\
$\sigma=5.23 \mathrm{~dB}$ & $\sigma=7.33 \mathrm{~dB}$ & $\sigma=4.13 \mathrm{~dB}$ & $\sigma=6.04 \mathrm{~dB}$ \\
\hline \hline
\end{tabular}

It can be observed, in Table 4.7, that for the three considered antennas a similar path loss exponent was obtained, although slightly higher for the Patch 2. The directional antenna present a lower standard deviation, $\sigma$, compared to the omnidirectional ones. This can be explained by the fact that the directive antenna focuses the power in one direction, minimizing the received contributions from the rest. Therefore, the measured values are less spreaded out, resulting in lower deviation from the logarithmic fitting model. Regarding the path loss exponent, looking at the measured path loss values for each antenna, shown in Figure 4.16, it can be observed that they are quite similar.

The fact that the path loss exponent is slightly lower for the directional antenna, it might be due to its specific radiation characteristics when used for in vivo measurements involving different types of tissues, since the antenna was designed to be primarily matched to human fat [73].

In order to estimate the $\left(x_{I B}, y_{I B}, z_{I B}\right)$ coordinates of the in-body antenna, the Non Linear Least Square method described in Section 3.3.2.3 was applied using different receivers combinations. Figure 4.17 and Figure 4.18 report the relative localization error evaluated through 4.5 and obtained by selecting an 
increasing number of receivers experiencing the highest level of received power, using the antenna related and general path loss model of Table 4.7.

It can be observed that localization results obtained with the antenna related and general path loss model are very close to each other. Moreover, since path loss values and derived path loss models present a similar trend, no antenna is outperforming the others in terms of localization accuracy.

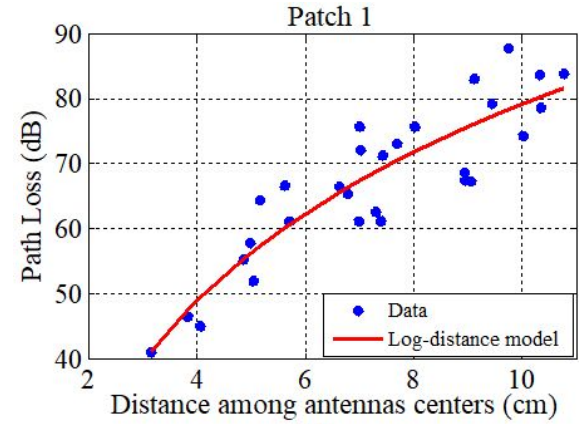

(a)

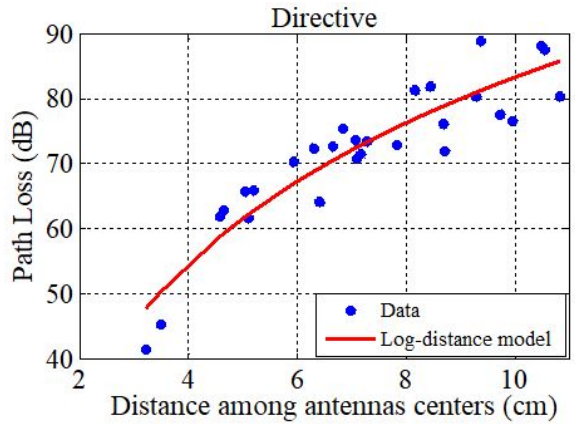

(b)

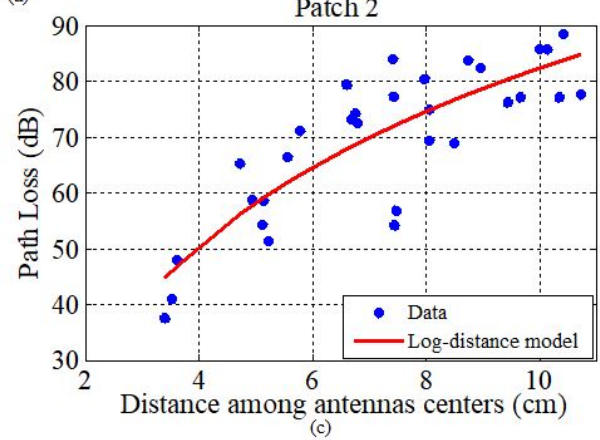

Figure 4.16: Measured path loss values and fitting model for Patch 1 (a), Directive (b) and Patch 2 (c) antenna

Considering the combination of receivers leading to the lowest relative error per in-body position, Table 4.8 and Table 4.9 report the obtained localization errors in $\mathrm{cm}$, using the antenna related and general PL models, respectively. Very similar errors were achieved, as previously pointed out, slightly higher in majority, when using the general PL model. 


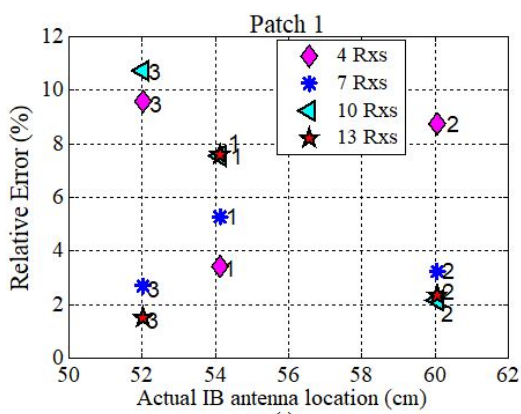

(a)

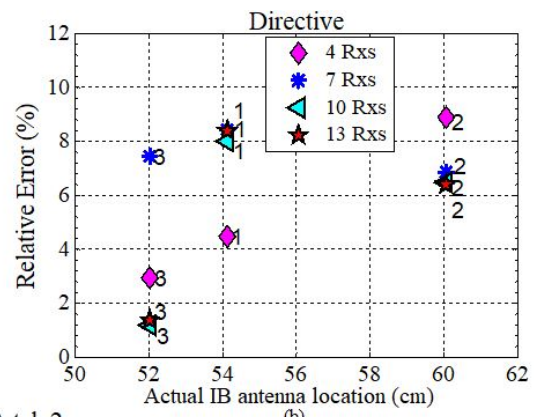

Patch 2

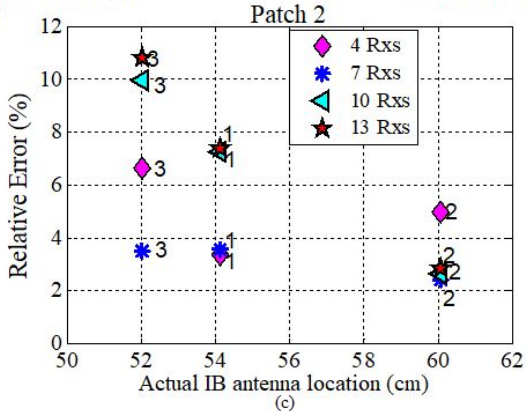

Figure 4.17: Relative localization error obtained with the antenna related path loss model for Patch 1 (a), Directive (b) and Patch 2 (c) antenna

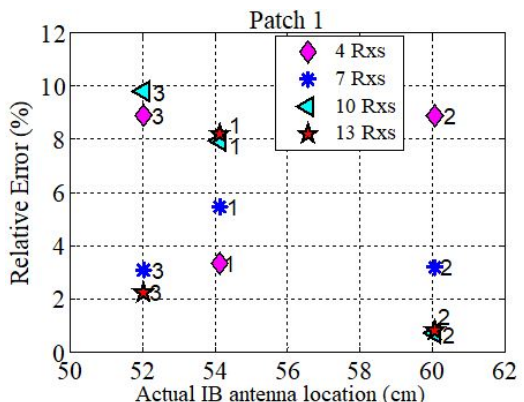

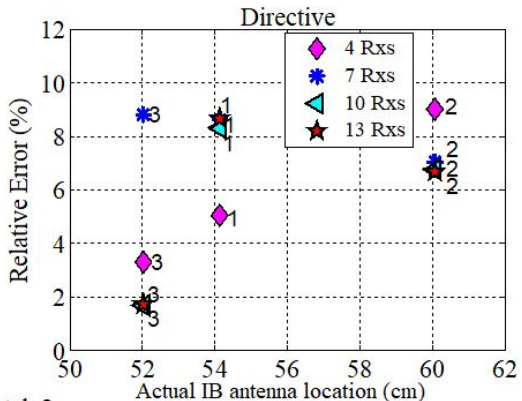

Patch 2

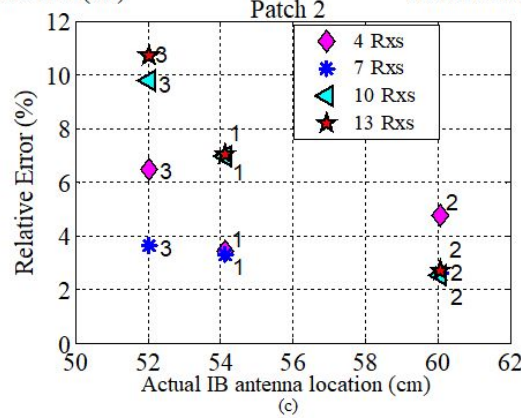

Figure 4.18: Relative localization error obtained with the general path loss model for Patch 1 (a), Directive (b) and Patch 2 (c) antenna 
Table 4.8: Localization errors for Patch 1, Patch 2, Directive antenna using antenna related PL models

\begin{tabular}{c|c|c|c}
\hline \hline Antenna related PL & Patch 1 & Patch 2 & Directive \\
\hline IB1 & $1.85 \mathrm{~cm}$ & $1.82 \mathrm{~cm}$ & $2.43 \mathrm{~cm}$ \\
IB2 & $1.30 \mathrm{~cm}$ & $1.45 \mathrm{~cm}$ & $3.84 \mathrm{~cm}$ \\
IB3 & $0.78 \mathrm{~cm}$ & $1.84 \mathrm{~cm}$ & $0.61 \mathrm{~cm}$ \\
\hline \hline
\end{tabular}

Table 4.9: Localization errors for Patch 1, Patch 2, Directive antenna using the general PL model

\begin{tabular}{c|c|c|c}
\hline \hline General PL & Patch 1 & Patch 2 & Directive \\
\hline IB1 & $1.81 \mathrm{~cm}$ & $1.79 \mathrm{~cm}$ & $2.73 \mathrm{~cm}$ \\
IB2 & $0.43 \mathrm{~cm}$ & $1.53 \mathrm{~cm}$ & $4.00 \mathrm{~cm}$ \\
IB3 & $1.16 \mathrm{~cm}$ & $1.91 \mathrm{~cm}$ & $0.87 \mathrm{~cm}$ \\
\hline \hline
\end{tabular}

\subsubsection{Hybrid RSS/AoA localization}

Considering the data collected during the third in vivo experiment, described in Section 3.2.4.4, an hybrid RSS/AoA scheme was used to exploit possible improvements compared to the RSS-based localization only. As a reminder, for these measurements, azimuth and elevation angle of the sensors attached to the in-body and on-body antenna were measured along with the distance between antennas and their coordinates with respect to the magnetic tracker's reference system (Figure 3.30). Path loss models were evaluated, using Equation 3.2, considering the path loss values measured by each antenna as well as considering the path loss values measured by the Patch 1 and CPW monopole antenna for in-body position 1 and by the Patch 2 and tear-shape monopole antenna for in-body position 2 . In the remainder of this section, results obtained using only the antenna related path loss models are presented, since better performance, in terms of localization error, were achieved, compared to those obtained using the path loss models per each pair of antennas. Table 4.10 reports the path loss model parameters, considering a reference distance, $d_{r e f}$, of $1 \mathrm{~cm}$ and a maximum distance between antennas of $12 \mathrm{~cm}$, for the four on-body antennas used in the experiment, per in-body position. From Table 4.10 it can be observed that for in-body position 1 the coplanar monopole antenna presents a higher path loss exponent and smaller standard deviation compared to the omnidirectional Patch 1, because of its directive radiation pattern. For in-body position 2, on the contrary, the omnidirectional Patch 2 shows a higher path loss exponent and smaller deviation than the directive tear-shape monopole. In general, limited measurements points were available to derive very accurate path loss models. Moreover, for the Patch 2 antenna, only seven on-body po- 
sitions were measured during the experiment, due to lack of time. Therefore, this most likely affected the derivation of the related path loss model.

Table 4.10: Path Loss Models for Patch 1, CPW monopole, Patch 2 and tear-shape monopole antenna

\begin{tabular}{c|c|c|c}
\hline \hline \multicolumn{2}{c|}{ IB1 } & \multicolumn{2}{c}{ IB2 } \\
\hline PL Patch 1 & PL CPW & PL Patch 2 & PL tear-shape \\
\hline$P L_{0, d_{r e f}}=18.53 \mathrm{~dB}$ & $P L_{0, d_{\text {ref }}=15.18 \mathrm{~dB}}$ & $P L_{0, d_{\text {ref }}}=2.84 \mathrm{~dB}$ & $P L_{0, d_{\text {ref }}=35.46 \mathrm{~dB}}$ \\
$n=5.79$ & $n=7.32$ & $n=8.66$ & $n=4.94$ \\
$\mu \sim 0$ & $\mu \sim 0$ & $\mu \sim 0$ & $\mu \sim 0$ \\
$\sigma=5.13 \mathrm{~dB}$ & $\sigma=4.22 \mathrm{~dB}$ & $\sigma=2.14 \mathrm{~dB}$ & $\sigma=4.56 \mathrm{~dB}$ \\
\hline \hline
\end{tabular}

In order to estimate the $\left(x_{I B}, y_{I B}, z_{I B}\right)$ coordinates of the in-body antenna, the method described in Section 3.3.2.3 was applied by minimizing the cost function in 3.21 through the Levenberg-Marquardt iterative algorithm. The weights $w_{i}$ in 3.21 were set to unity as no a priori knowledge of the estimation errors standard deviation was assumed.

Figure 4.19 reports the relative localization errors, evaluated as in Equation 4.5, for in-body position 1, using the Patch 1 and the coplanar waveguide monopole antenna employing the RSS (a) and the RSS/AoA (b) method. An increasing number of receivers, from 4 up to 10, experiencing the highest level of received power was used for the localization.

Figure 4.20 reports the relative localization errors, evaluated as in Equation 4.5, for in-body position 2, using the Patch 2 and the tear-shape monopole antenna employing the RSS (a) and the RSS/AoA (b) method. Since for the Patch 2 antenna only seven on-body locations were measured, the relative localization error, for the sake of comparison, was evaluated for both antennas considering an increasing number of receivers, from 4 up to 7 , experiencing the highest level of received power. Localization performed by fusing RSS and AoA information showed a similar or worse accuracy compared to the RSS method only as it can be observed in Figure 4.19 and Figure 4.20. The reason can be linked to the fact that, due to the considered in-body locations, azimuth and elevation angles between the in-body antenna and the on-body receivers have similar values. Table 4.11 shows, as example, the azimuth and elevation angles between in-body position 1 and the Patch 1 antenna for the different measured on-body locations (Figure 3.27). Since the values are close to each other, when performing localization the set of angle measurements do not refine enough the space resulting from the intersection of the spheres, defined from the range measurements. Similar behavior was observed for the same in-body position, considering the CPW monopole antenna and for in-body position 2 considering the Patch 2 and the tear-shape monopole antenna. 
Compared to the RSS approach only, using the angle information increases the complexity of the cost function to minimize (3.21). If the measured angle values are not sufficiently diversified the minimization of the cost function will be more time consuming, since the number of variables is higher, and the accuracy will be the same or less because not enough information is given to restrict the search space of the in-body position to estimate.

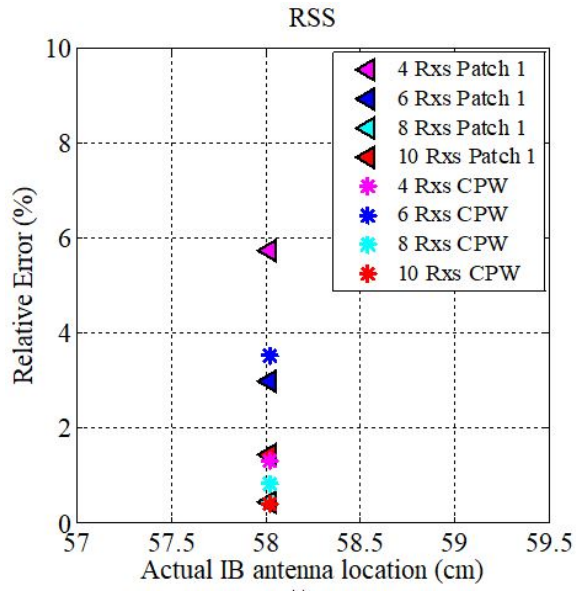

(a)

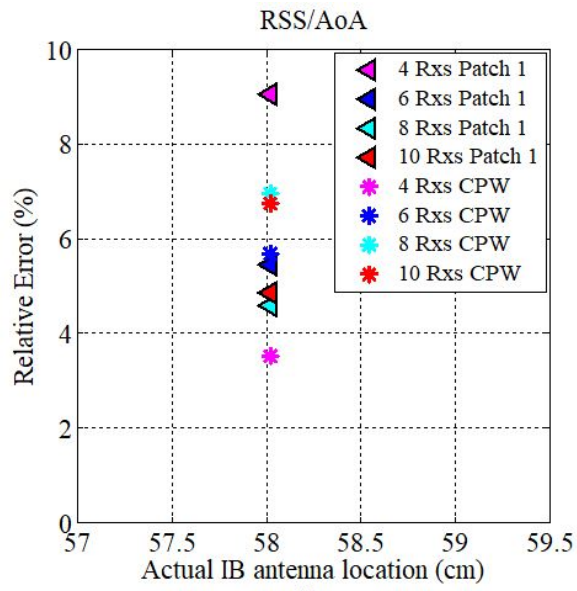

(b)

Figure 4.19: Relative localization error for in-body position 1 obtained using the Patch 1 and CPW monopole antenna for RSS (a) and RSS/AoA (b) approach

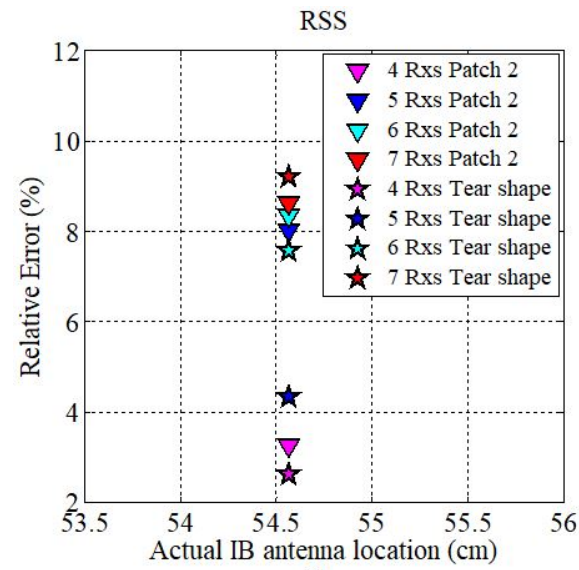

(a)

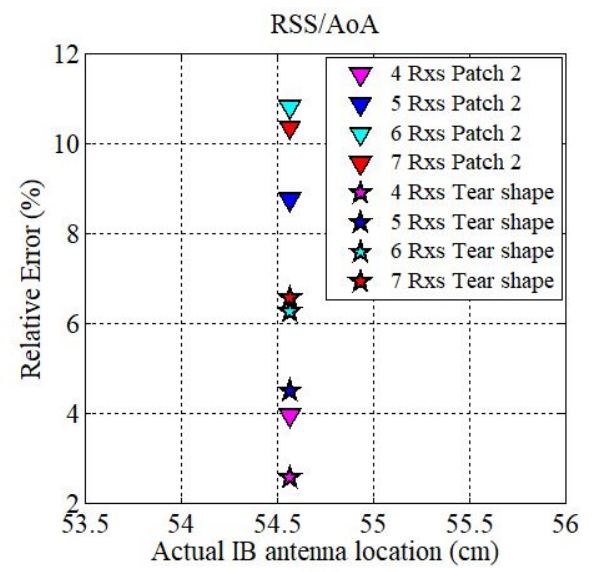

(b)

Figure 4.20: Relative localization error for in-body position 2 obtained using the Patch 2 and tear-shape monopole antenna for RSS (a) and RSS/AoA (b) approach 
Table 4.11: Azimuth and elevation angles in degrees between in-body position 1 and Patch 1 antenna for the ten measured on-body locations

\begin{tabular}{|c|c|c|}
\hline N Rxs & Azimuth & Elevation \\
\hline $\mathrm{Rx} 1$ & -36.96 & -4.93 \\
\hline $\mathrm{Rx} 2$ & -44.32 & -5.17 \\
\hline $\mathrm{Rx} 3$ & -44.06 & -2.75 \\
\hline $\mathrm{Rx} 4$ & -38.52 & -4.53 \\
\hline $\mathrm{R} \times 5$ & -39.83 & -5.62 \\
\hline $\mathrm{Rx} 6$ & -47.27 & -6.21 \\
\hline $\mathrm{Rx} 7$ & -41.95 & -5.66 \\
\hline $\mathrm{Rx} 8$ & -43.02 & -5.79 \\
\hline $\mathrm{Rx} 9$ & -40.00 & -3.97 \\
\hline $\mathrm{Rx} 10$ & -35.50 & -2.12 \\
\hline
\end{tabular}

In terms of localization error, evaluated as in Equation 4.4, for in-body position 1 , the lowest error corresponding to $0.23 \mathrm{~cm}$ was obtained through the RSS method using the directive CPW monopole antenna and 10 receivers, as can be inferred from Figure 4.19. For in-body position 2, the lowest error corresponding to $1.43 \mathrm{~cm}$ was obtained through indistinctly the RSS or RSS/AoA method using the tear-shape monopole antenna and 4 receivers, as can be observed in Figure 4.20.

\subsection{Effect of Receivers Distribution on Localiza- tion Accuracy}

As detailed in the previous sections, two-dimensional and three-dimensional RSS-based localization was performed by selecting the receivers experiencing the highest level of received power. This is in line with the way current WCE localization algorithms work [82], [84]. For 2D positioning, using the heterogeneous phantom-based setup, in Section 4.3.1.2, the lowest localization error was obtained by the combination of three receivers experiencing the highest level of received power. For $2 \mathrm{D}$ positioning, using the homogeneous phantom-based setup, in Section 4.3.1.1, the lowest error was obtained by the combination of receivers achieving the lowest average ranging error.

For 3D positioning, using software simulations and five receivers, in Section 4.4.1.1, for in-body location at $7 \mathrm{~cm}$ the lowest error was obtained, again, by the combination of receivers achieving the lowest average ranging error. For 3D positioning, using in vivo measurements in Section 4.4.1.2 and using simulations considering nine receivers in Section 4.4.1.1, increasing the number of receivers experiencing the highest level of received power not always improved the localization accuracy. 


\subsection{Effect of Receivers Distribution on Localization Accuracy}

Thus, the selection of receivers experiencing the highest power as well as increasing the number of receivers, used for positioning, not necessarily leads to the best localization precision. There is a tendency suggesting that by selecting the receivers combination achieving the lowest average ranging error better accuracy could be obtained [85].

In the remainder of this section, a deeper investigation of this trend is carried out, considering the results obtained through simulations, laboratory measurements and in vivo experiments presented in the previous sections.

Considering the simulation results shown in Figure 4.13, Table 4.12 and Table 4.13, report for each in-body position under study the relative localization error and the average ranging error obtained with different number of receivers, experiencing the highest level of received power [86].

Table 4.12: Localization error and average ranging error for in-body position 1 (IB1) to in-body position 4 (IB4) obtained from simulations

\begin{tabular}{c|cc|cc|cc|cc}
\hline \hline \multirow{2}{*}{ N Rxs } & \multicolumn{2}{|c|}{ IB1 $(4 \mathrm{~cm})$} & \multicolumn{2}{c|}{ IB2 $(5 \mathrm{~cm})$} & \multicolumn{2}{c|}{ IB3 $(6 \mathrm{~cm})$} & \multicolumn{2}{c}{ IB4 $(6.3 \mathrm{~cm})$} \\
& LE & Avg & LE & $A v g_{R E}$ & LE & Avg & LE & Avg \\
& $(\%)$ & $(\mathrm{cm})$ & $(\%)$ & $(\mathrm{cm})$ & $(\%)$ & $(\mathrm{cm})$ & $(\%)$ & $(\mathrm{cm})$ \\
\hline 4 & 17.46 & 0.06 & 18.72 & 0.10 & 21.12 & 0.12 & 4.28 & 0.08 \\
5 & 30.56 & 0.07 & 29.05 & 0.11 & 31.91 & 0.11 & 9.12 & 0.10 \\
6 & 16.36 & 0.06 & 18.13 & 0.11 & 22.01 & 0.10 & 2.82 & 0.10 \\
7 & 14.69 & 0.06 & 17.39 & 0.10 & 21.74 & 0.09 & 2.14 & 0.09 \\
8 & 14.36 & 0.06 & 16.63 & 0.10 & 21.05 & 0.09 & 8.64 & 0.10 \\
9 & 14.40 & 0.06 & 16.91 & 0.10 & 21.78 & 0.11 & 8.82 & 0.16 \\
\hline \hline
\end{tabular}

Table 4.13: Localization error and average ranging error for in-body position 5 (IB5) to in-body position 7 (IB7) obtained from simulations

\begin{tabular}{|c|c|c|c|c|c|c|}
\hline \multirow[b]{2}{*}{ N Rxs } & \multicolumn{2}{|c|}{ IB5 $(7 \mathrm{~cm})$} & \multicolumn{2}{|c|}{ IB6 $(7.2 \mathrm{~cm})$} & \multicolumn{2}{|c|}{ IB7 $(8 \mathrm{~cm})$} \\
\hline & $\begin{array}{l}\mathrm{LE} \\
(\%)\end{array}$ & $\begin{array}{c}A v g_{R E} \\
(\mathrm{~cm})\end{array}$ & $\begin{array}{l}\mathrm{LE} \\
(\%)\end{array}$ & $\begin{array}{c}A v g_{R E} \\
(\mathrm{~cm})\end{array}$ & $\begin{array}{l}\mathrm{LE} \\
(\%)\end{array}$ & $\begin{array}{c}A v g_{R E} \\
(\mathrm{~cm})\end{array}$ \\
\hline 4 & 17.23 & 0.05 & 15.32 & 0.53 & 17.62 & 0.45 \\
\hline 5 & 21.67 & 0.07 & 12.30 & 0.55 & 20.32 & 0.47 \\
\hline 6 & 15.08 & 0.08 & 11.42 & 0.49 & 16.62 & 0.46 \\
\hline 7 & 14.97 & 0.09 & 12.56 & 0.43 & 16.66 & 0.47 \\
\hline 8 & 13.72 & 0.11 & 17.29 & 0.53 & 15.62 & 0.47 \\
\hline 9 & 13.66 & 0.10 & 16.80 & 0.52 & 16.27 & 0.43 \\
\hline
\end{tabular}

For all seven in-body positions an increment in the average ranging error mostly result in a higher localization error and vice versa. However, this is true not for all cases reported in Table 4.12 and Table 4.13. In fact, for in-body position at $6 \mathrm{~cm}$ (IB3), for example, passing from 4 to 5 receivers the average ranging error slightly decreases and the localization error increases. The same happens 
for in-body position at $8 \mathrm{~cm}$ (IB7) passing from eight to nine receivers. The opposite behavior is observed for in-body position at $7.2 \mathrm{~cm}$ (IB6) where an increment in the average ranging error results in a lower localization error, passing from 4 to 5 receivers.

The behavior of the localization error, taking as example in-body antenna position IB3 and IB6 can be explained by looking, in Figure 4.21, at the dispersion of the path loss values, corresponding to the selected four/five receivers used for positioning, with respect to the log-distance fitting model.

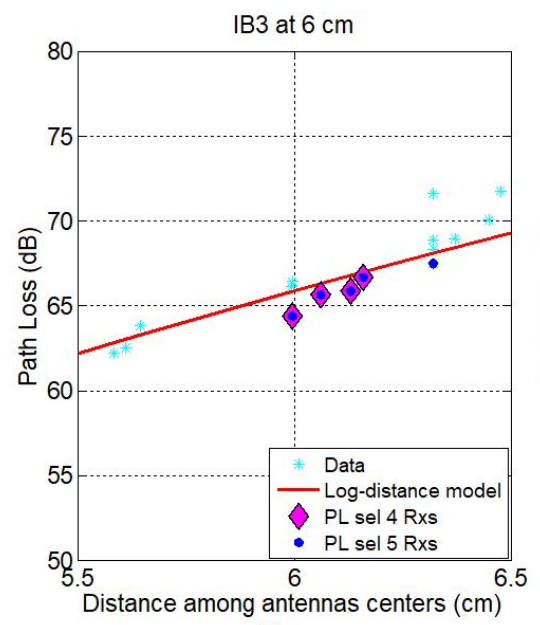

(a)

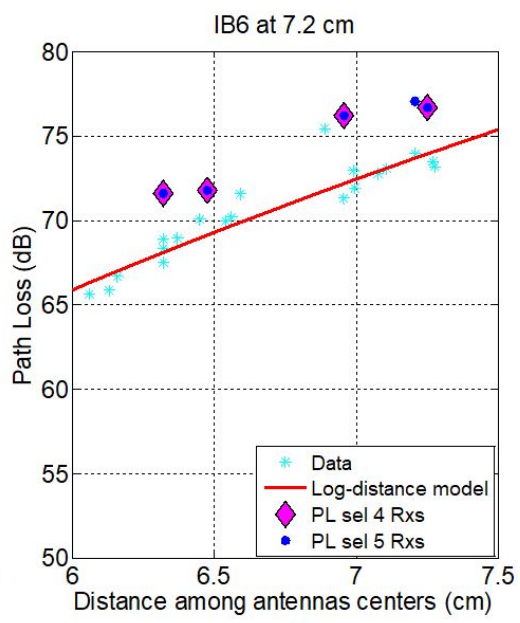

(b)

Figure 4.21: Simulated path loss values and fitting model along with path loss values of selected receivers for in-body position at $6 \mathrm{~cm} \mathrm{(a)} \mathrm{and} \mathrm{at} 7.2 \mathrm{~cm} \mathrm{(b)}$

For in-body position IB3 (Figure 4.21(a)), passing from four (magenta diamond) to five receivers (blue dots) the average ranging error decreases because the selected path loss values are all in close proximity. Since they are not uniformly distributed around the distance to estimate $(6 \mathrm{~cm})$ adding one more receiver does not increase the diversity of the estimated ranging distances (all values are very close to each other) used for localization. For in-body position at $7.2 \mathrm{~cm}$, (Figure 4.21(b)), adding one more receiver increases the diversity of the estimated ranging distances used for localization as the selected path loss values are more evenly distributed around the distance to estimate $(7.2 \mathrm{~cm})$.

Considering the first in vivo experiment results, detailed in Section 4.4.1.2.1, Table 4.14 reports for the two in-body positions under study the relative localization error and the average ranging error obtained with different number of receivers, experiencing the highest level of received power. The number of 


\subsection{Effect of Receivers Distribution on Localization Accuracy}

receivers is limited to ten so none of them is outside $(>8 \mathrm{~cm})$ the region of validity of the derived path loss model.

Table 4.14: Localization error and average ranging error for in-body position 1 (IB1) and in-body position 2 (IB2) obtained from first in vivo experiment

\begin{tabular}{c|cc|cc}
\hline \hline \multirow{2}{*}{ N Rxs } & \multicolumn{2}{|c|}{ IB1 } & $(4.67 \mathrm{~cm})$ & \multicolumn{2}{c}{ IB2 } & $(4.46 \mathrm{~cm})$ \\
LE & $\begin{array}{c}\text { Avg } \\
(\mathrm{cm})\end{array}$ & $\begin{array}{c}\text { LE } \\
\text { (\%) }\end{array}$ & $\begin{array}{c}A v g_{R E} \\
(\mathrm{~cm})\end{array}$ \\
\hline 4 & 1.91 & 0.85 & 4.61 & 0.69 \\
5 & 1.99 & 1.04 & 3.50 & 0.77 \\
6 & 1.79 & 0.89 & 1.10 & 0.69 \\
7 & 1.82 & 0.93 & 1.42 & 0.87 \\
8 & 1.94 & 0.85 & 1.30 & 0.79 \\
9 & 1.51 & 0.78 & 1.38 & 0.83 \\
10 & 1.48 & 0.77 & 1.40 & 0.89 \\
\hline \hline
\end{tabular}

Table 4.14 shows that for both in-body positions an increment or decrement in the average ranging error mostly result in higher or lower localization errors, respectively. However, this is not true for all the reported cases. For in-body position 1, passing from seven to eight receivers, the average ranging error decreases but the localization error slightly increases. As previously done for the simulation results to explain this trend, the dispersion of the path loss values, corresponding to the selected seven/eight receivers used for positioning, with respect to the log-distance fitting model is reported in Figure 4.22 for in-body position 1 .

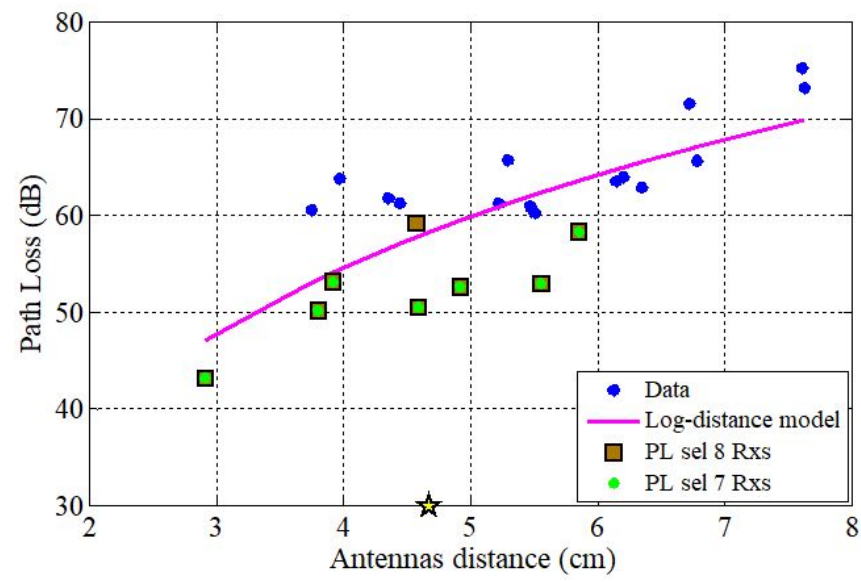

Figure 4.22: In vivo measured path loss values and fitting model along with path loss values of selected receivers for in-body position 1 
The yellow star on $\mathrm{x}$-axis corresponds to the true location of in-body position 1, reported in Table 4.14, calculated with respect to the central receiver Rx5 in Figure 3.19.

It can be observed in Figure 4.22 that passing from seven (green asterisks) to eight (brown squares) receivers the average ranging error decreases as the additional eighth selected value is close to the fitting curve resulting in a small error. The localization error slightly increases because the eighth estimated ranging distance is very similar to the one estimated for a true distance of $5.9 \mathrm{~cm}$. Therefore, it does not provide additional diversified information to restrict the search space of the minimum of the squares of the errors on the distance (3.18).

The trend of the localization error was also investigated for 2D positioning in order to verify whether the minimization error algorithm, used to evaluate in $3 \mathrm{D}$ the in-body antenna coordinates is the main cause of the behavior observed for the simulations and in vivo measurements. In this case, as a reminder, the in-body antenna coordinates were evaluated by solving a linear system in two unknown, using three receivers so the localization error does not depend on the optimization algorithm.

With the aim of comparing the tendency of the localization error in both cases (2D and 3D), 2D localization was performed using the heterogeneous phantom-based setup described in Section 3.2.2.3 for similar antenna distances and positions. To this end, the in-body antenna locations corresponding to the grid points which are more aligned with respect to the central on-body receiver (Rx1 in Figure 3.15) were considered. Table 4.15 reports for each in-body position under study the 2D localization error along with the average ranging error, for the three considered combinations of receivers, the same used to evaluate the results considering more grid points (Section 4.3.1.2).

Table 4.15: 2D localization error and average ranging error for in-body position 1 (IB1) to in-body position 3 (IB3) obtained from heterogeneous phantom-based measurements

\begin{tabular}{|c|c|c|c|c|c|c|}
\hline \multirow[b]{2}{*}{ Rxs } & \multicolumn{2}{|c|}{ IB1 $(5.5 \mathrm{~cm})$} & \multicolumn{2}{|c|}{ IB2 $(6.4 \mathrm{~cm})$} & \multicolumn{2}{|c|}{ IB3 $(7.3 \mathrm{~cm})$} \\
\hline & $\begin{array}{l}\mathrm{LE} \\
(\%)\end{array}$ & $\begin{array}{c}A v g_{R E} \\
(\mathrm{~cm})\end{array}$ & $\begin{array}{l}\mathrm{LE} \\
(\%)\end{array}$ & $\begin{array}{c}A v g_{R E} \\
(\mathrm{~cm})\end{array}$ & $\begin{array}{l}\mathrm{LE} \\
(\%)\end{array}$ & $\begin{array}{c}A v g_{R E} \\
(\mathrm{~cm})\end{array}$ \\
\hline 2,3 (ref), 4 & 4.05 & 0.31 & 1.12 & 0.24 & 3.21 & 0.26 \\
\hline 1 (ref), $, 3,4$ & 4.74 & 0.21 & 3.66 & 0.12 & 4.35 & 0.16 \\
\hline 2 (ref) $, 3,5$ & 6.33 & 0.27 & 1.60 & 0.28 & 7.04 & 0.34 \\
\hline
\end{tabular}

It can be observed, in Table 4.15, that for all the considered in-body positions the lowest error is achieved using the combination of receivers 2, 4 and 3, taken as reference. This combination of receivers, as pointed out in Section 4.3.1.2, is experiencing on average, per in-body position, the highest level of received power. Nevertheless, the corresponding average ranging error per in- 


\subsection{Effect of Receivers Distribution on Localization Accuracy}

body position, is not the lowest. The lowest average ranging error is indeed achieved by the combination of receivers 3,4 and 1 taken as reference. This tendency of the localization error is the same observed for the $3 \mathrm{D}$ case, therefore the minimization algorithm is not the main cause of this behavior. As for the $3 \mathrm{D}$ case, the dispersion of the path loss values, corresponding to the selected combinations of receivers, with respect to the fitting model curve was analyzed and is depicted in Figure 4.23. The selected path loss values, used for ranging estimation, are grouped per in-body position and are represented for receivers 2, 3 (reference), 4 in Figure 4.23(a), for receivers 1 (reference), 3, 4 in Figure 4.23(b) and for receivers 2 (reference), 3, 5 in Figure 4.23(c), respectively. The yellow stars on the $\mathrm{x}$-axis illustrates the actual positions of the in-body antenna to estimate.

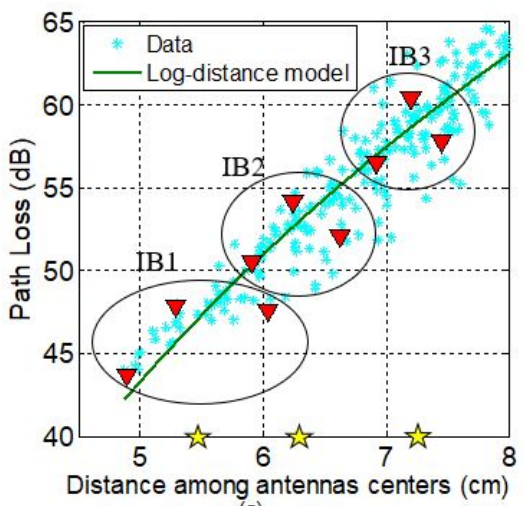

(a)

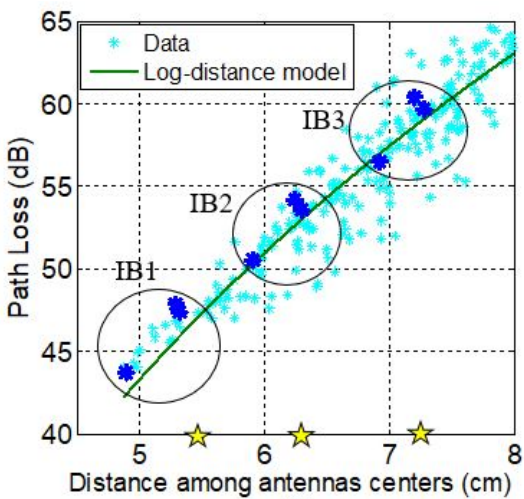

(b)

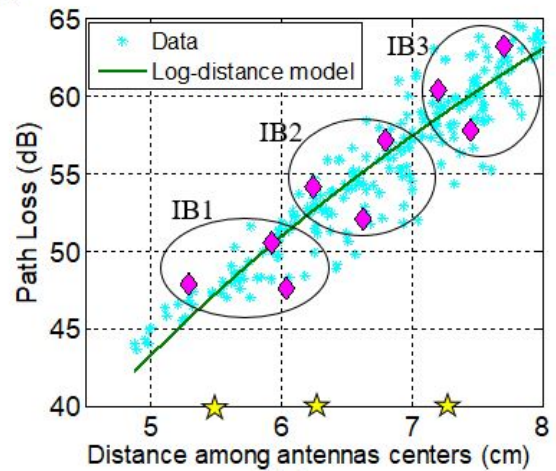

(c)

Figure 4.23: Laboratory measured path loss values and fitting model along with path loss values of selected receivers for in-body positions under study 
Looking at Figure 4.23, same behavior as for the 3D case is observed. Using the combination of receivers 2, 3 (reference) and 4 (Figure 4.23(a)) the selected path loss values used for ranging estimation are more uniformly distributed around the distance to estimate, compared to the other combinations of receivers (Figure 4.23(b) and Figure 4.23(c)). This means more diversity among the estimated ranging distances values, used for localization. Using the combination of receivers 1 (reference), 3 and 4, (Figure 4.23(b)), the average ranging error is minimized but two of the selected path loss values are very close to each other, leading to similar estimates of the ranging distance.

It is important to point out that, even not using the minimization algorithm, the coordinates estimation through the linearized system of equations (Equation 3.17) still depends on the estimates of the ranging distances corresponding to the selected receivers used for localization.

These results point out that the average ranging error metric alone is not enough to ensure that the localization error should increase or decrease. As a matter of fact, the distribution of the selected path loss values around the in-body distance to estimate also affects the localization accuracy.

\subsection{Summary and Discussion}

Summarizing the results, it was found that Time of Arrival (ToA) method presents higher inaccuracy compared to the RSS-based method. This mainly was related to the bandwidth resolution used in the measurements as well as to the approximation model of the propagation velocity when considering more than one human tissue (heterogeneous phantom). Regarding the bandwidth, it is important to mention that above $5.1 \mathrm{GHz}$ the $\mathrm{RF}$ signal propagating through human tissues suffers from severe attenuation. Therefore, increasing the frequency band would not be a good option, for this kind of in-body to on-body applications.

Performance of RSS-based method heavily depends on the derived path loss model and also on the selected receivers used for localization.

Regarding the path loss, models with different fitting parameters were presented in the previous sections. Regardless the difference in the parameters values, it is important to point out that simulated and measured path loss were compared and validated. Figure 4.24 reports the path loss values obtained for the multilayer phantom-based setup (Figure 3.2.2.3), those obtained through simulations using the setup in Figure 3.17 and those obtained during the first in vivo experiment (Section 3.2.4.2). Path loss obtained with the multilayer phantom container were on average 2 to $3 \mathrm{~dB}$ higher than those obtained with homogeneous muscle phantom, in section 3.2.2.2.1 due to the 
extra losses introduced by the $2 \mathrm{~cm}$ fat layer. Moreover, since in simulations and in in vivo experiments the abdominal fat was less than $2 \mathrm{~cm}$, with the aim of fair comparison, path loss values obtained with the multilayer phantom were reasonably shifted $2 \mathrm{~cm}$ to the left, in Figure 4.24 .

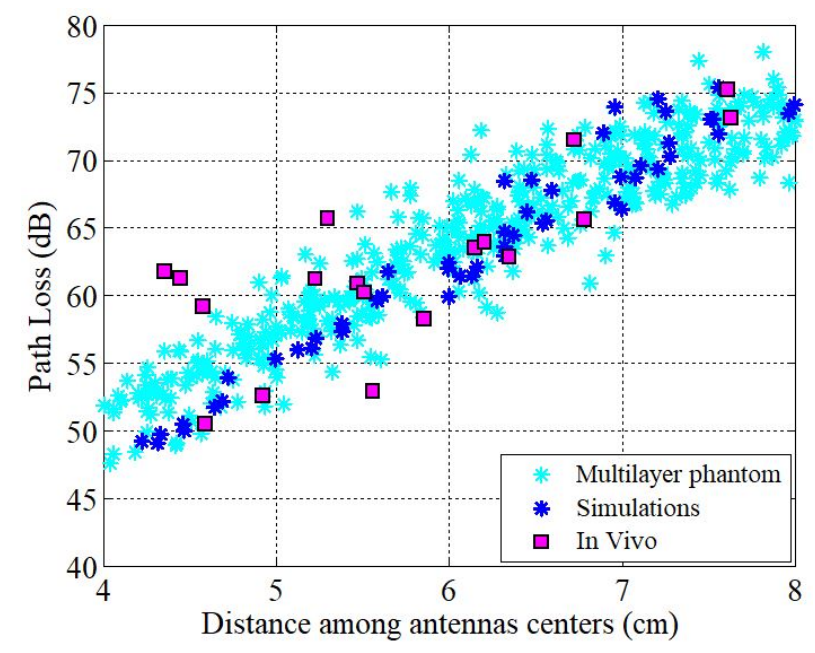

Figure 4.24: Comparison between path loss values obtained through laboratory measurements, simulations and first in vivo experiment

The comparative between path loss values obtained with the three different methodologies, all involving more than one human tissue, shows a high level of agreement.

Regarding the receivers selection, for both two-dimensional and three-dimensional positioning the same behavior for the localization error was observed. By only selecting the receivers experiencing the highest level of received power or by selecting only those achieving the lowest average ranging error is not sufficient to maximize the accuracy of the localization. The combination of both criteria might help improving the precision of the localization, although the distribution of the selected path loss values around the distance to estimate will still have an impact on the accuracy. Combining both criteria will set a bound on the number of receivers used for localization, as the selected ones need to satisfy the highest level of received power as well as the lowest average ranging error condition. Having as few sensors as possible on the abdomen is important for the comfort of the patient in real capsule endoscopy applications. Nevertheless, in order to jointly apply these two criteria, the real distance between the capsule and the external receiving sensors needs to be known at anytime, 
which makes it infeasible.

In terms of localization error, for the three methodologies, similar values were obtained with the RSS approach, assuming ideal and not ideal channel estimation. For simulations and in vivo measurements slightly lower localization errors were obtained for a few in-body locations, when considering not ideal channel estimation. This mainly depended on the derived path loss model and on the distribution of the selected path loss values, corresponding to the receivers used for localization, with respect to the fitting model curve and to the distance to estimate.

For two-dimensional positioning, considering homogeneous and heterogeneous phantom-based measurements average localization errors between $0.72 \mathrm{~cm}$ and $1.6 \mathrm{~cm}$ were obtained. For three-dimensional positioning, using simulations, error values between $0.5 \mathrm{~cm}$ and $1.43 \mathrm{~cm}$ were obtained. For three-dimensional positioning considering all the performed in vivo measurements, errors between $0.23 \mathrm{~cm}$ and up to $4 \mathrm{~cm}$ were achieved.

Performance obtained with RSS/AoA approach were similar to or worse than those obtained with RSS approach only. Since the orientations of the onbody receivers with respect to the in-body antenna were very similar to each other, no additional information was provided to reduce the searching space of the in-body position to estimate, through the minimization algorithm.

Directive on-body antennas did not outperform, in general, the omnidirectional ones. Only for two-dimensional positioning, considering homogeneous phantom-based measurements, and for few in vivo in-body locations a slightly lower localization error was obtained. For localization purposes, the efficiency of the antenna used is relevant in terms of path loss model and of the maximum distance which can be covered between in-body and on-body antenna before the received power goes below the noise level. However, a small standard deviation between measured path loss values and fitting model curve can not ensure the best localization performance. As a matter of fact, the positioning accuracy also depends on the selected receivers and on the distribution of their corresponding path loss values around the distance to estimate. 


\section{Chapter 5}

\section{Real-Case Application: HemoPill Localization}

In this chapter, experimental measurements conducted during a secondment at Ovesco Endoscopy company in Tübingen, Germany are presented. Measurements were carried out using a robot assisted system available at the hosting company with the aim of localizing a wireless capsule used in digestive endoscopy as a possible side application for this kind of device. The operating frequency, in this case, is not Ultra-wideband but narrowband ISM band, as the capsule was designed for this specific frequency band, which is one of the approved and commonly used for medical applications.

The first section describes the wireless capsule and its functionality. In the second section, the measurements setup is detailed. Finally, the third section is devoted to explain and discuss the obtained localization results.

\subsection{Detection of Upper Gastrointestinal bleed- ing: HemoPill}

\subsubsection{Introduction}

Upper gastrointestinal bleeding refers to bleeding from the esophagus, stomach, or duodenum. It is one of the most frequent emergency in endoscopy units with high clinical relevance, due to its high incidence (50-70,000 hospital admissions per year in the United Kingdom and 300,000 in the United States) [87]. Acute upper gastrointestinal bleedings from ulcers or esophago-gastric varices are life threatening medical conditions which require immediate endoscopic therapy. 
Elderly patients and those with chronic medical diseases incurring in acute GI bleeding have a higher risk of death [87].

Peptic ulcers accounts for approximately $35 \%$ of cases with a lethality of 7 to $10 \%$. Bleeding occurs when the ulcer erodes into an underlying artery. Acute bleeding caused by stress ulcers is more though to occur. These kind of ulcers are common among patients under high levels of physical stress such as those in intensive care units. Stress ulcers can be life-threatening because they tend to affect very sick people and the related bleeding is usually severe and develops in multiple sites.

Esophageal varices are abnormal, enlarged veins in the esophagus. This condition occurs most often in people with serious liver diseases. Variceal bleeding is usually life-threatening with a mortality of $25-30 \%$ [88].

The key for successful treatment of acute upper gastrointestinal bleeding is early recognition. Any delay in the detection may lead to significant blood loss and increases the risk for the patient. Upper endoscopy should be undertaken and is recommended within 24 hours from the discovery in most patients with acute upper GI bleeding to confirm diagnosis and possibly treat any underlying lesion. Endoscopic treatment leads to initial hemostasis in most cases, but the recurrence rates are quite high. About 25\% (and up to 70\%) hospitalized for variceal bleeding experiences a rebleeding episode during the first six weeks after initial bleeding control. The rebleeding rate for peptic ulcers is about 15$35 \%$ [89], [90]. Most recurrent bleeding occurs within 72 hours and is associated with a relatively high risk of death.

Thus, early recognition of bleeding and rebleeding would reduce the mortality. Emergency endoscopy is not always available due to logistic or lack of personnel. The use of Video Capsule Endoscopy (VCE) in emergency room was also investigated for the detection of acute GI bleeding [91], [92]. Results showed a potential reduction of the time to emergency endoscopy and therapeutic intervention. Despite the promising results, the possibility of missing lesions in the fundus and other less accessible areas is a limitation of VCE. Moreover, it is difficult to train emergency doctors or specialists for interpretation and set up of VCE in the emergency room [93].

Due to the necessity of early recognition, Ovesco Endoscopy company developed a sensorized telemetric capsule, the HemoPill, which immediately indicates any bleeding in the upper gastrointestinal tract without the need for time-consuming and elaborate endoscopy [4]. The device was tested and validated through pre-clinical studies [94], [95] and recently obtained the Conformité Européenne (CE) mark. In the following section, the capsule and its functionality are described. 


\subsubsection{Design and Working Principle}

The HemoPill system, shown in Figure 5.1 comprises a sensor capsule that can either be swallowed to detect active bleeding in the esophagus and stomach or anchored endoscopically to the wall of the gastrointestinal tract, close to a potential bleeding source for continuous monitoring and surveillance. The capsule has a size of $6.5 \mathrm{~mm}$ in diameter and $25.5 \mathrm{~mm}$ in length (Figure 5.1). It is battery-powered and contains an optical sensor for detecting the presence of blood and a telemetry unit (transceiver chip, helical antenna) that sends measurement values to an extra-corporeal receiver. The telemetry unit is a transmitter which operates in the frequency band of around $433 \mathrm{MHz}$ and sends data packets including implant ID, operation status, and digitalized sensor values.
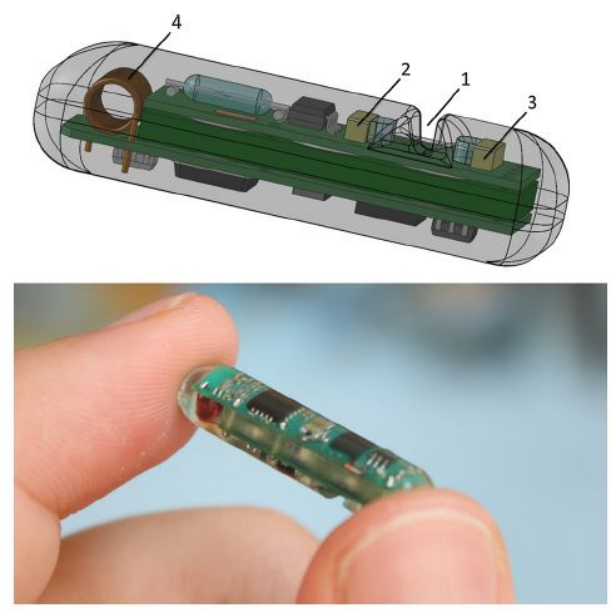

Figure 5.1: CAD model and photo of the bleeding sensor capsule [4] 1. recess for the entry of blood, 2. photo transistor, 3. LEDs, 4. helical antenna. Dimension of the capsule: 6.5 $\mathrm{mm}$ in diameter, $25.5 \mathrm{~mm}$ in length.

The working principle of the bleeding sensor is based on ratiometric intensity measurements of the characteristic optical properties of blood [4]. Blood, indeed, presents high absorption of violet light, while red light is comparatively well transmitted. This optical property is used for the sensor: at a wavelength of approximately $415 \mathrm{~nm}$ (violet), the transmission of light through blood reaches a minimum and is up to three orders of magnitude lower than the transmission at a wavelength of $720 \mathrm{~nm}$ (red, maximum transmission within measurement range), depending on the optical density of the blood sample. According to these optical characteristics of blood, the quotient of the measured intensity of red light divided by the measured intensity of violet light is 
used as single indicator value to predict the presence of blood in the sensor recess. Thus, the quotient increases with decreasing violet intensity indicating a higher concentration of blood.

The optical sensor is a ratiometric intensity-based sensor, designed to be miniaturized for integration into a swallowable or implantable capsule. The aim is comparing the transmission of light at $415 \mathrm{~nm}$ to the transmission of light at $720 \mathrm{~nm}$. For this purpose, the implant cast provides a recess, through which light from two LEDs of the respective wavelengths is transmitted sequentially (Figure 5.1). The recess is dimensioned to allow fluids to flow into the optical pathway of the sensor. The remaining intensity of the light after its travel through the recess is measured by a photo transistor. This allows the implant to calculate the ratio between the violet and red light.

\subsection{Measurements Setup}

\subsubsection{Ovesco RAMCE System}

Measurements were conducted using a Robot Assisted Magnetic Capsule Endoscopes (RAMCE) system [5], available at the company and designed for colonoscopy applications. This system was primarily developed to overcome one common limit of wireless capsule endoscopy: the absence of active capsule control as most commercially available WCEs rely on peristalsis (passive locomotion) to traverse the GI tract. This reduces the diagnostic accuracy especially in the stomach and in the colon where the capsule trajectory is uncontrollable and unpredictable. Moreover, it can lead to capsule retention, or late capsule excretion resulting in an incomplete visualization of the bowel wall.

Figure 5.2 shows the RAMCE system available at the company.

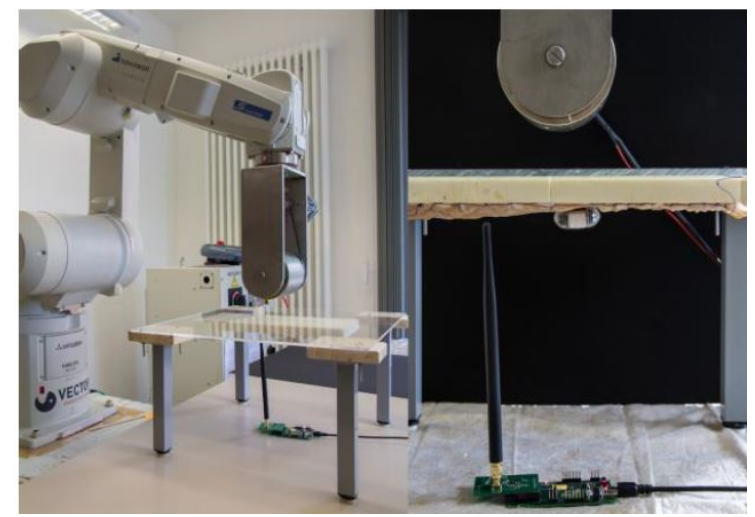

Figure 5.2: External system and testbed available at Ovesco [5] 
It consists of a capsule and an external system that acts as a receiver and an actuator. RAMCE operates on the principle of magnetic attraction between the capsule that hosts an onboard permanent magnet and an external magnetic field produced by a permanent magnet which is mounted on a robotic arm which moves the magnet. The interaction between the two magnetic fields results in capsule movement.

The capsule prototype has a dimension of $38 \mathrm{~mm}$ in length and of $18.5 \mathrm{~mm}$ in diameter. It was designed to operate at $868 \mathrm{MHz}$ with a working time of 40 minutes, as a screening colonoscopy lasts on average 30 to 45 minutes [96]. The architecture was designed to easily replace the Silver Oxide batteries which are used as a power source for the capsule electronics. The onboard permanent magnet is axially polarized and rated at 1.43 Tesla, with a volume of 230.4 $\mathrm{mm}^{3}$.

The capsule is actuated by a cylindrical external permanent magnet of $80 \mathrm{~mm}$ in length and $90 \mathrm{~mm}$ in diameter (Figure 5.2), coupled to a stepper motor that can rotate the magnet using a gear assembly. This assembly is mounted on a 6 degrees of freedom robotic arm effectively providing 7 degrees of freedom to the external magnet. The robot controller and the stepper motor are connected to a PC that runs a control software allowing manipulation of the robot to $1 \mathrm{~mm}$ and $1^{\circ}$ accuracy on all axes. The external system also consists of a transceiver operating at $868 \mathrm{MHz}$, connected to the $\mathrm{PC}$ that receives the data transmitted by the capsule. The PC control software logs this data and displays it in a Graphical User Interface (GUI).

\subsubsection{HemoPill Testbed}

Experimental measurements were carried out at Ovesco to perform RSS-based localization of the HemoPill, described in Section 5.1.2 and shown in Figure 5.1. This could be considered as a side application for this kind of ingestible device, where the physician could apply an array of sensors to the patient (sort of the same type of those used for WCE procedure in Figure 2.1) in order to locate the pill, in case of retention or if needed. Besides the potential impact of this side application, this work also intended to contribute to the lack of existing studies on RF-based localization in the $433-434 \mathrm{MHz}$ frequency band. Available literature mainly rely on wireless capsule endoscopy [45], [97] and either the presence of the body is not properly taken into account [45] or no localization error is reported [97]. Therefore, it was interesting and of relevance carrying out some experimental measurements to exploit the RSSbased approach for ingestible devices operating in the ISM band. With the aim of collecting data for positioning, the RAMCE system detailed in the previous section was used. Since localization of the pill through the received signal strength was the main goal, the robotic arm permanent magnet was removed 
and replaced by a wooden pole to support and move the external receiver in several different positions, as depicted in Figure 5.3(b).

In order to emulate the real case scenario, where the pill is located in the stomach, a muscle-like phantom was prepared according to the recipe in [98]. The liquid is a mixture of water, sugar and salt and the ingredients percentages refer to its preparation in the MICS frequency band. Since the relative permittivity of the muscle tissue at MICS band (on average 57.1) and at 433-434 $\mathrm{MHz}$ (on average 56.9) is very similar [63] and considering the fact that the ingredients were easy-to-find, compared to other recipes available in literature, the mixture in [98] was used.

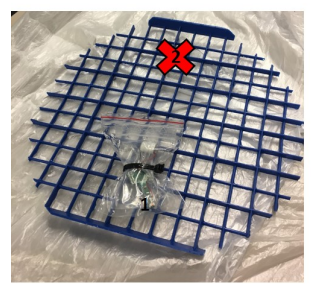

(a)

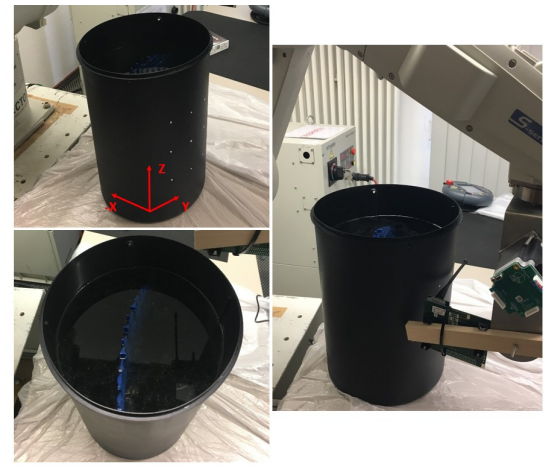

(b)

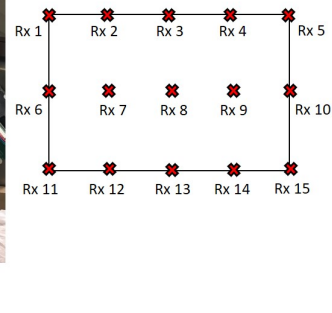

(c)

Figure 5.3: HemoPill measured positions (a), measurements setup (b) and measured receiving points (c)

The muscle phantom was then poured inside a cylindrical container of sizes comparable to those of the human torso (Figure 5.3(b)). The capsule was fixed on a plastic circular grid, to avoid uncontrolled fluctuations, in two different positions (Figure 5.3(a)) and then immersed in the cylindrical container filled up with phantom (Figure 5.3(b)). The receiver, mounted on the robotic arm, was moved in different locations along $\mathrm{x}, \mathrm{y}, \mathrm{z}$ axis with grid size of $(\mathrm{Ny}=5, \mathrm{Nz}=3$, $\mathrm{Nx}$ slightly varies for each point), as depicted in Figure 5.3(b) and 5.3(c). In order to emulate the real in-body to on-body scenario, the receiving antenna was placed as closer as possible to the edge of the cylindrical container (Figure 5.3(b)). For each in-body to on-body position, the received signal strength was measured 100 times. Then, the average RSS was calculated. The in-body helical antenna as well as the receiving antenna present an omnidirectional radiation pattern. Measurements, with the same setup, same capsule and same receiving positions shown in Figure 5.3, were conducted twice, on different days. 


\subsection{RSS-based Localization Results}

For both measurement campaign, path loss models were derived from the measured data as no standardized models are available in literature for in-body to on-body communications at ISM (433-434 MHz) frequency band. For each in-body to on-body position the path loss was evaluated as in [99]:

$$
P L(d B)=G_{R}+P_{T}-P_{R}
$$

where $P_{T}$ is the transmitting power equal to $0 \mathrm{dBm}, P_{R}$ is the receiving power in $\mathrm{dBm}$ and $G_{R}$ is the gain of the receiving antenna which is equal to $2 \mathrm{dBi}$. Path loss values were then fitted through a log-distance model as in Equation 3.2 , considering a reference distance, $d_{\text {ref }}$, equal to $1 \mathrm{~cm}$. Figure 5.4 depicts the measured data along with the obtained fitting models and Table 5.1 reports the related path loss parameters for the two conducted measurement campaigns.
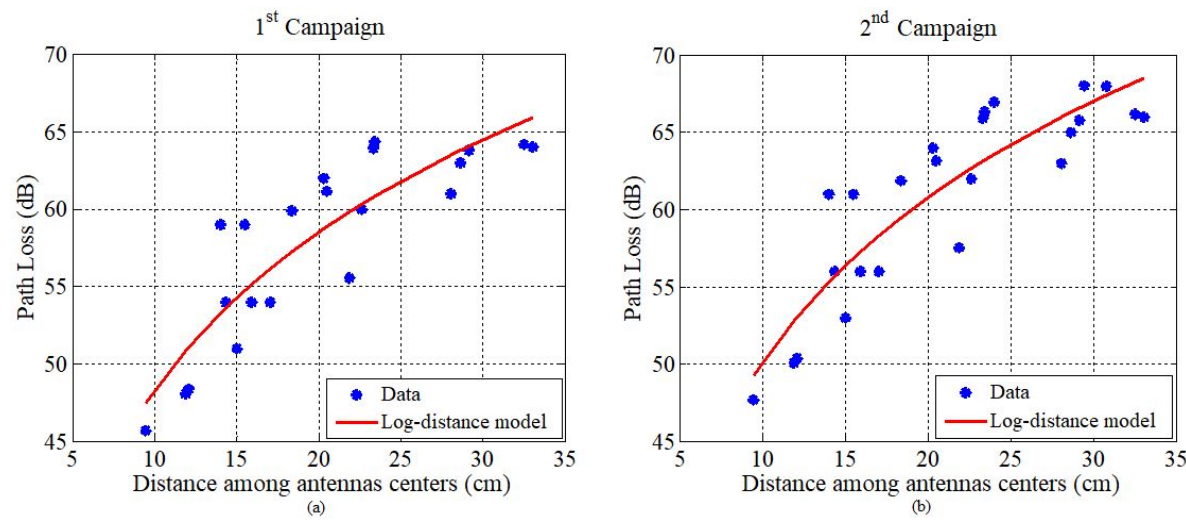

Figure 5.4: Measured data along with log-distance fitting model for the $1^{\text {st }}$ (a) and $2^{\text {nd }}$ measurement campaigns

Table 5.1: Path Loss Models for $1^{\text {st }}$ and $2^{\text {nd }}$ measurement campaigns

\begin{tabular}{c|c}
\hline \hline $1^{\text {st }}$ campaign & $2^{\text {nd }}$ campaign \\
\hline$P L_{0, d_{\text {ref }}}=14.80 \mathrm{~dB}$ & $P L_{0, d_{\text {ref }}}=23.84 \mathrm{~dB}$ \\
$n=3.54$ & $n=3.60$ \\
$\mu \sim 0$ & $\mu \sim 0$ \\
$\sigma=2.78 \mathrm{~dB}$ & $\sigma=4.08 \mathrm{~dB}$ \\
\hline \hline
\end{tabular}




\section{CHAPTER 5. REAL-CASE APPLICATION: HEMOPILL LOCALIZATION}

The fitting models obtained for the two measurement campaigns, as can be observed in Figure 5.4 and Table 5.1 present a very similar behavior. For the second measurements campaign, compared to the first one, the RSS readings were a bit lower and the standard deviation of the path loss values with respect to the fitting model a bit higher. This is due to the fact that for the second campaign, the distance between the HemoPill and the on-body receivers was on average slightly higher $(1-1.5 \mathrm{~cm})$ with respect to the first campaign. The derived fitting models in Figure 5.4 shows that there is a loss between 4 and $7 \mathrm{~dB}$ for an increase of $5 \mathrm{~cm}$ in distance. Considering the standardized path loss models for MICS band [40] these results are quite close to those obtained for deep tissue implant to body surface, taking into account that the frequency band used is slightly different and no full body model, including all organs and tissues, was used.

Through the derived path loss models, ranging distance was estimated for each transmitting-receiving position as in Equation 3.5 and the location of the HemoPill evaluated using the receivers experiencing the highest level of receiving power (lowest path loss). The Non Linear Least Square (NLLS) method described in Section 3.3.2.3 was implemented as in 3.18 to estimate the coordinates of the capsule.

For both measurement campaigns and both in-body positions, the estimated coordinates were plotted versus the real ones in a two-dimensional view, as shown in Figure 5.5, as it would be in a real case scenario where the physician looking at the torso section of the patient on a screen could visually detect the position of the HemoPill inside the stomach, if needed.

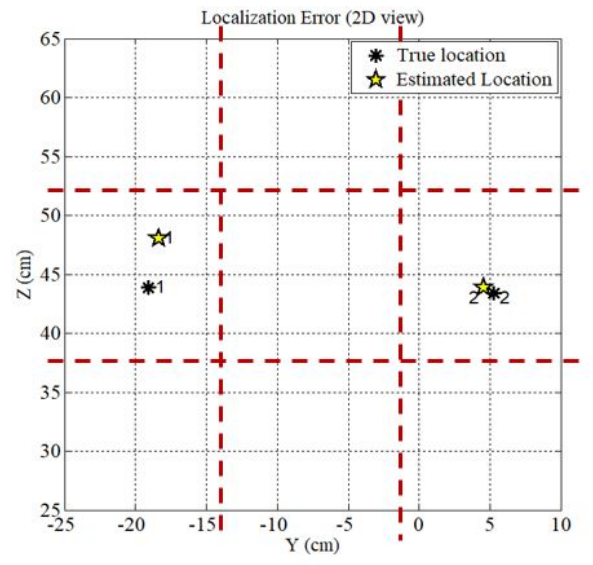

(a)

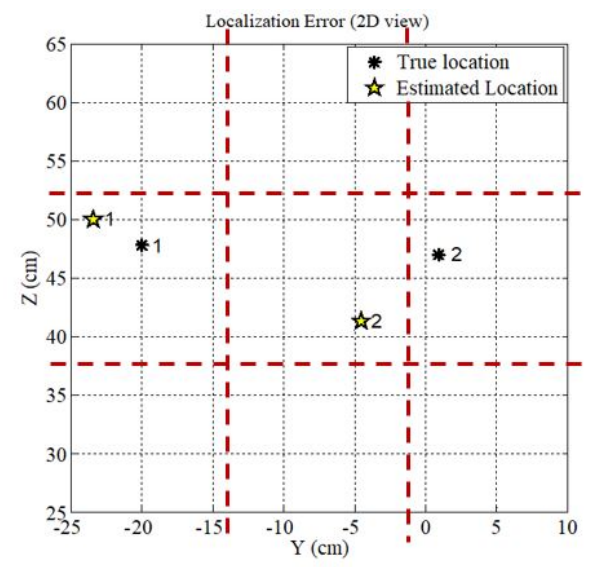

(b)

Figure 5.5: Estimated location vs real location of HemoPill for the first measurements campaign (a) and for the second measurement campaign (b) 
Table 5.2 reports the localization error in $\mathrm{cm}$, calculated as in Equation 4.4, along with the absolute error on the estimation of the x-coordinate of the Hemopill, for both measurement campaign and both in-body locations, using the combination of receivers leading to the best accuracy.

Table 5.2: Localization error and error on $\mathrm{x}$ estimation for the first and second measurement campaign

\begin{tabular}{c|ccc|ccc}
\hline \hline \multirow{2}{*}{ IB pos } & \multicolumn{3}{|c|}{$1^{\text {st }}$ campaign } & \multicolumn{3}{c}{$2^{\text {nd }}$ campaign } \\
& & $\begin{array}{c}\text { LE } \\
(\mathrm{cm})\end{array}$ & $\begin{array}{c}\text { Err on } \mathrm{x} \\
(\mathrm{cm})\end{array}$ & $\mathrm{N}$ Rxs & $\begin{array}{c}\text { LE } \\
(\mathrm{cm})\end{array}$ & $\begin{array}{c}\text { Err on } \mathrm{x} \\
(\mathrm{cm})\end{array}$ \\
\hline 1 & 7 & 6.52 & 5.14 & 9 & 4.31 & 0.43 \\
2 & 5 & 2.39 & 2.19 & 8 & 8.30 & 2.44 \\
\hline \hline
\end{tabular}

Results in Figure 5.5 shows that the location of the HemoPill can be estimated to be more or less in one of the quadrants of the torso section, i.e. upper (left, center, right) center (left, center, right), bottom (left, center, right). However, it can be observed in Table 5.2 that the accuracy on average needs to be improved. Except for the estimated position 2 (Figure 5.5(a)), for the first measurements campaign, which presents a localization error of $2.15 \mathrm{~cm}$, using five receivers, for all the other position estimates the error is above $5 \mathrm{~cm}$. This is caused, as shown in Figure 5.6, by the high spread between the path loss values corresponding to the receivers selected for localization with respect to the fitting model curve.
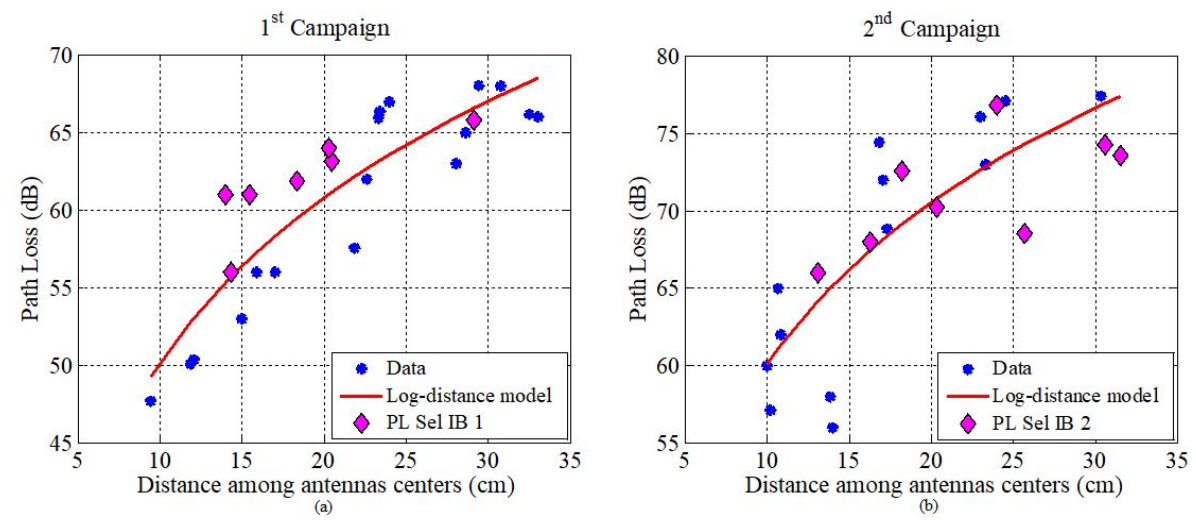

Figure 5.6: Selected path loss values for in-body position 1 for the first measurements campaign (a) and for in-body position 2 for the second measurements campaign (b)

Figure 5.6 reports the selected path loss values for ranging estimation, related to the number of receivers indicated in Table 5.2 for in-body position 1 , 
considering the first measurements campaign (a) and for in-body position 2 considering the second measurements campaign (b), as they present the highest localization errors. The spread between the selected path loss values and the fitting model curve causes significant ranging errors resulting in poor localization accuracy. As a matter of fact an average ranging error of $3.30 \mathrm{~cm}$ was obtained for in-body position 1 of the first campaign (Figure 5.6(a)) and of $4.29 \mathrm{~cm}$ for in-body position 2 of the second campaign (Figure 5.6(b)).

In this case, besides the limited number of measured points, due to time constraints, to derive a more accurate path loss model, the available receiving antenna was probably not the optimal one for this specific application. 


\section{Chapter 6}

\section{Conclusions and Future Trends}

Wireless capsule endoscopy is an effective, simple, painless method of visualizing the GI tract that has numerous potential applications and an increasing role in digestive pathological examination. However, multiple improvements are needed to overcome the limitations of the current capsule endoscopy procedures which include poor image quality and the difficulty of localizing anomalies in the small bowel or colon.

The current standard for in-body communications, IEEE 802.15.6, only allows the use of narrow band systems. Even though current wireless medical devices have relatively good penetration through human tissues and certain robustness, high data rate wireless connections are not possible given the limited available bandwidth. To solve this issue, UWB technology has emerged in the latest years as a potential candidate for the revision of this standard to improve implanted communications qualitatively.

For the localization process, using the RF signal employed for image transmission to also locate the capsule is preferable in order to reduce the complexity of the capsule's hardware and to preserve, in this way, its battery lifetime.

At present, RF-based localization for capsule endoscopy at UWB frequencies has been limitedly explored in literature. This thesis intended to contribute to the lack of these studies. Particularly, the adequateness of existing radio frequency based localization approaches for indoor applications was explored when applied to WCE scenario considering Ultra-Wideband technology as communication interface. Such techniques imply a previous characterization of the UWB in-body to on-body propagation channel. In order to carry out these tasks, three main methodologies were used: software-based simulation using digital human models, laboratory measurements involving homogeneous 


\section{CHAPTER 6. CONCLUSIONS AND FUTURE TRENDS}

and heterogeneous liquid phantom models and in vivo measurements using porcine models. RSS, ToA and hybrid RSS/AoA techniques were investigated. Positioning results, including the impact of the position and the number of selected receivers on the localization accuracy, were analyzed and compared for the three methodologies used.

The main contributions of this thesis are summed up in Section 6.1, whereas proposed future works are detailed in Section 6.2.

\subsection{Conclusions}

This thesis was focused on contributing to the lack of results related to UWB RF-based localization for wireless capsule endoscopy. Particularly, this work intended to give a comprehensive view of how much accuracy can be obtained in more realistic scenarios compared to software-based simulations only, which constitute the most commonly used approach in the existing literature.

Chapter 3 described the three different methodologies used to collect data for positioning. In order to reproduce the real in-body to on-body WCE scenario for localization purposes, a small UWB patch antenna, formerly designed to operate inside the human body, was used as transmitting in-body source, since at present there no capsule endoscopes operating at these frequencies. Several different types of receiving on-body antennas, formerly designed to operate on the surface of the human body were employed for the measurements. Particularly, the receiving antenna was moved in different on-body locations in order to emulate the sensors array used in current WCE procedures to locate the capsule.

Besides the software-based simulations, a dedicated measurement setup for in-body to on-body communications, developed at UPV facilities, was employed along with the liquid phantom models used to mimic the permittivity of the fat and human muscle. In vivo experiments were conducted as well, at the Hospital La fe, in Valencia, in order to test the developed localization techniques in a scenario which is the closest to the reality. The in-body and on-body antennas used for the different measurement campaigns showed good matching within the UWB frequency band of interest. For all the three methodologies used to collect data, except for the third in vivo experiment, the orientation of the in-body and on-body antenna, as far as possible, was kept the same to better investigate the effect of the propagation channel on the localization accuracy.

RSS, ToA and hybrid RSS/AoA approaches were employed to perform onedimensional, two-dimensional and three-dimensional localization. For RSSbased approaches, in order to estimate the ranging distance, path loss models were derived from the measurements, as in literature there are currently no standardized models for in-body to on-body communications at UWB fre- 
quencies. For ToA-based method and heterogeneous body model, the average propagation velocity of the RF signal was evaluated through a weighted sum of the signal velocity across each of the considered human tissues. For hybrid RSS/AoA approach, the ranging distance was evaluated through the derived path loss model and the azimuth and elevation angle were measured by the magnetic tracker employed in the measurements.

In order to evaluate the coordinates of the in-body antenna, for $2 \mathrm{D}$ positioning a linearized approach was used along with different combinations of three receivers. For $3 \mathrm{D}$ positioning the non linear least square method was employed where the sum of the squares of the errors on the distance was minimized through the Levenberg-Marquardt algorithm.

Chapter 4 presented the obtained localization results. Considering the RSS-based approach, better performance were obtained, in general, using the path loss model related to the specific on-body antenna used for localization.

For 1D positioning, the localization error, as expected, was increasing as the distance among antennas increased. The best accuracy was achieved for the locations where in-body and on-body antenna were almost aligned. For these positions an average localization error between 0.06 and $1.7 \mathrm{~cm}$ was obtained in the estimation of $\mathrm{x}$ and $\mathrm{y}$-coordinate of the in-body antenna.

For 2D positioning the lowest error was obtained using the combination of three receivers experiencing either the highest level of received power (heterogeneous phantom case) or the lowest average ranging error (homogeneous phantom case). An increment of $1 \%$ in the localization error was observed when assuming not ideal channel estimation, i.e. in case of considering a real receiver capable of detecting only few multipath components of the propagation channel, as it usually receives for a given period of time.

The on-body directive Vivaldi antenna, employed in the homogeneous phantom measurements, performed slightly better than the omnidirectional one. This was linked to the fact that, when using the Vivaldi antenna, the path loss values corresponding to the best combination of receivers were closer to the fitting model curve, resulting in lower ranging errors and consequently lower localization errors compared to the omnidirectional antenna.

In terms of localization error, considering homogeneous and heterogeneous phantom-based measurement campaigns, average errors between $0.72 \mathrm{~cm}$ and $1.6 \mathrm{~cm}$ were obtained.

For 3D positioning similar results were obtained assuming ideal and not ideal channel estimation. For several in-body positions the localization error was decreasing when considering not ideal channel estimation. This mainly depended on the derived path loss model and on the distribution of the path loss values, corresponding to the receivers selected for localization, with respect to the fitting model curve and to the distance to estimate. Directive on-body 
antennas did not outperform, in general, the omnidirectional ones. Only for few in vivo in-body locations the localization error was slightly lower. As a matter of fact, most likely due to the limited number of measurement points, all antennas presented similar path loss models.

Performance obtained using the hybrid RSS/AoA approach were similar to or slightly worse than those obtained with RSS approach only. This was due to the fact that the orientations of the on-body receivers with respect to the in-body antenna were very similar to each other, resulting in no additional information to refine the searching space of the in-body position to estimate.

In terms of localization error, considering the conducted software-based simulations, errors between $0.5 \mathrm{~cm}$ and $1.43 \mathrm{~cm}$ were obtained. Considering all the performed in vivo experiments, errors between $0.23 \mathrm{~cm}$ and up to $4 \mathrm{~cm}$ were achieved.

For both $2 \mathrm{D}$ and $3 \mathrm{D}$ positioning results showed that the selection of receivers experiencing the highest level of received power as well as increasing the number of those receivers not always led to the best performance. As a matter of fact, the precision of the localization depended on both the average ranging error and on the distribution of the path loss values related to the selected receivers with respect to the distance to estimate.

Considering the ToA approach, for both 1D and 2D positioning higher localization errors were obtained due to bandwidth resolution and possibly to the approximation model used to evaluate the average propagation velocity, when more than one human tissue was considered (heterogeneous phantom case). For this reason, ToA approach was not further used for 3D localization.

As a general conclusion, results obtained from simulations, laboratory measurements and in vivo experiments showed that designing RSS-based localization techniques for capsule endoscopy using the low UWB frequency band is feasible and practical. Acceptable accuracy could be obtained using eight (as the sensors array currently used for WCE localization) or less on-body receivers. As a matter of fact, increasing the number of receivers, in general, was not the best solution to improve the localization performance.

The accuracy of the path loss model used for ranging estimation plays a key role in achieving high precision positioning. Therefore, it is important to perform extensive measurement campaigns, using suitable testbeds and antennas, in order to characterize as best as possible the in-body to on-body propagation channel.

Chapter 5 presented experimental measurements conducted during a research period at Ovesco Endoscopy company in Tübingen, Germany. Measurements were carried out using a robot assisted system available at the company with the aim of localizing a wireless capsule used to detect upper GI bleeding. 
Localization was considered as a side application for this kind of ingestible device, where the physician could apply an array of sensors to the patient (sort of the same type used for WCE procedure) in order to locate the pill, in case of retention or if needed.

The operating frequency in this case was not Ultra-Wideband but narrowband ISM (433-434 MHz), since the pill was designed to operate at these frequencies. In order to emulate the real case scenario, where the pill is located inside the stomach, a muscle-like phantom suitable for the frequency band of interest was prepared. Measurements were conducted by moving the external receiver through the robotic arm in different locations on the surface of the phantom cylindrical container whose sizes were comparable to those of the human torso. Results obtained using the RSS-based approach showed that the capsule could be visually located in a two-dimensional view, as it would be in reality when the physician is looking at the torso section of the patient on a screen. However, except for one in-body position which presented a localization error of $2.39 \mathrm{~cm}$, for the other three considered in-body locations errors were above $4 \mathrm{~cm}$. The high spread between the path loss values corresponding to the receivers selected for localization and the fitting model curve caused significative ranging errors, resulting in poor localization accuracy. This was mainly due to the fact that limited measurement points were available to derived a more accurate path loss model. Furthermore, the available receiving antenna was probably not the optimal one for this specific application.

\subsection{Future Directions}

This thesis constitutes a first step in the testing of RF-based localization techniques for UWB capsule endoscopy applications in more realistic environments compared to software simulations only. There is, without doubts, a significant amount of work that needs to be done to improve the current WCE localization algorithms.

An accurate characterization of the in-body propagation channel, as pointed out in this thesis, is of fundamental importance for localization using the received signal strength. UWB technology has emerged in the latest years as possible candidate for next generation wireless capsule endoscopy. Currently, no standard regulates UWB in-body to on-body communications. In this dissertation several path loss models, derived from the measurements, were presented. A good level of agreement was assessed between simulations, laboratory measurements and in vivo experiments. However, the derived models are not valid for antennas distance above $8 \mathrm{~cm}$ or $11 \mathrm{~cm}$ (second in vivo experiment) due to the specific measurement setup used. Therefore, there is a need in the future to perform more extensive measurement campaigns in order to better characterize the IB2OB UWB propagation channel and to derive a more ac- 


\section{CHAPTER 6. CONCLUSIONS AND FUTURE TRENDS}

curate and general path loss model which could be adopted for these kind of communications.

In this thesis, except for the lastin vivo experiment, the orientation of the in-body and on-body antenna was kept the same, in order to better investigate the effect of the propagation channel on the localization performance. In real applications, this condition is not satisfied at all and the unknown orientation of the capsule inside the GI tract affects the localization accuracy. As part of the future work, further experimental measurements needs be performed to take into account the misorientation between antennas. As a matter of fact, the directionality (or null) in the radiation pattern of the transmitting antenna could be exploited to estimate its orientation through several on-body receivers, as presented in a recent study in [100]. Additionally, a possible solution to overcome signal losses, due to orientation changes of the in-body antenna, could be the use of circularly polarized in-body antennas which are less vulnerable to polarization mismatches and multipath distortion [101].

For the scenarios considered in this thesis, localization using the received signal strength showed in general an acceptable accuracy, even if not for all the measured in-body positions. However, there is still much room for improvement. In order to enhance the localization accuracy, the RSS method could be combined with a motion model extracted from the images taken by the capsule endoscope. These informations are usually combined by means of a Kalman or particle filter which is used to predict the capsule's position. These classes of algorithms are known as Simultaneous Localization and Mapping (SLAM) [52]. These hybrid localization techniques along with the adoption of UWB systems, could provide accurate, smooth and continuous localization and mapping results that meet the requirements of WCE applications. 


\section{Appendix A}

\section{Graphical User Interface (GUI)}

In this appendix a GUI developed to visualize the obtained localization results is presented. To this end the Matlab platform was used as developing tool. Results related to the first and second in vivo measurement campaigns reported in Section 4.4.1.2.1 and in Section 4.4.1.2.2 were considered as they are the closest to the reality, compared to simulations and laboratory measurements. In order to have a realistic view of 3D positioning, a model of the small bowel was designed and included in the GUI.

Figure A.1 shows how the GUI appears once the related script file is launched in the Matlab command window, as initial step. The upper left panel, Setup Parameters, includes all the parameters the user can select to visualize in a second step the localization results, depending on which experiment is considered. The Localization panel includes the buttons needed to visually view the $3 \mathrm{D}$ positioning results inside a human small bowel model in the Visualize Results panel as well as in a new figure window or in a text file as a report.

The first step, in order to use the GUI, is selecting the type of experiment (first or second in vivo) in the Setup Parameters panel. If the second in vivo is chosen, the popup menu Antenna Type is activated as shown in Figure A.3 and one on-body antenna among Patch 1, Patch 2 and Directive can be selected. For the first in vivo the same popup menu is deactivated as shown in Figure A.2 since this experiment was carried out using only the Patch 1 as on-body antenna (Section 3.2.4.2). Depending on the chosen experiment on the right panel of the GUI the related measurement setup is displayed as depicted in Figure A.2 and A.3. Next, the in-body position to visualize need to be selected in the Setup Parameters panel. For the first in vivo position 1, position 2 or 


\section{APPENDIX A. Graphical User Interface (GUI)}

both of them can be chosen. For the second in vivo, position 1, position 2, position 3 or all of them can be selected.

Once all the parameters have been set, the buttons in the Localization panel are activated as shown in Figure A.4.

In order to visualize the 3D localization results within the GUI, inside the small bowel model displayed in Visualize Results panel, the button Show 3D Position must be pressed. Figure A.5 and Figure A.6 show how the 3D localization looks like when pressing the Show 3D Position button considering an example related to the first and second in vivo experiment, respectively. The estimated selected in-body positions are represented by magenta triangles while the real locations in yellow stars. Positioning is performed by selecting the best combination of receivers leading to lowest localization error, for both measurement campaigns, based on the results presented in Section 4.4.1.2.1 and in Section 4.4.1.2.2. If the user is interested in having more detailed information regarding the localization parameters and the errors committed in the in-body positions estimation, by pressing the button Localization Error Report, a report file is generated as shown in Figure A.7 considering the example in Figure A.5. The text file includes a summary of the chosen parameters along with the receivers selected per in-body position, the adopted localization criteria and the relative and absolute localization errors committed in the estimation of the in-body location as well as the tracking error passing from position 1 to position 2, calculated as in Equation 4.11 and Equation 4.12, respectively. The real and estimated in-body locations inside the small bowel model can be better visualized by pressing the Open Results Figure button which opens the results displayed in the Visualize Results panel in a new figure window, as depicted in Figure A.8 for the example of Figure A.5. This way, the user can interact with the figure using the matlab plot tools to move and rotate it in order to better view where the estimated and real in-body positions are situated inside the small bowel.

It is important pointing out that the in vivo measurements were performed inside the abdomen of the porcine and not inside any of its internal organs, particularly the small bowel. Even if hypothetically performed inside the pig's small bowel, the anatomy between the human small bowel and the pig's one is different. Nevertheless, the GUI was developed with the aim of visualizing in the most realistic way the $3 \mathrm{D}$ position of an in-body source, in the way the physicians would like to experience in real WCE procedures. Graphically, the positions of the in-body antenna inside the small bowel are just an example of possible locations of the capsule endoscope in real applications. The real and estimated positions of the in-body antenna were scaled and adapted to fit inside the small bowel model in order to visually appreciate (as much as possible) how much error in the estimation of the real position the doctors would have to deal with in a real case scenario. 


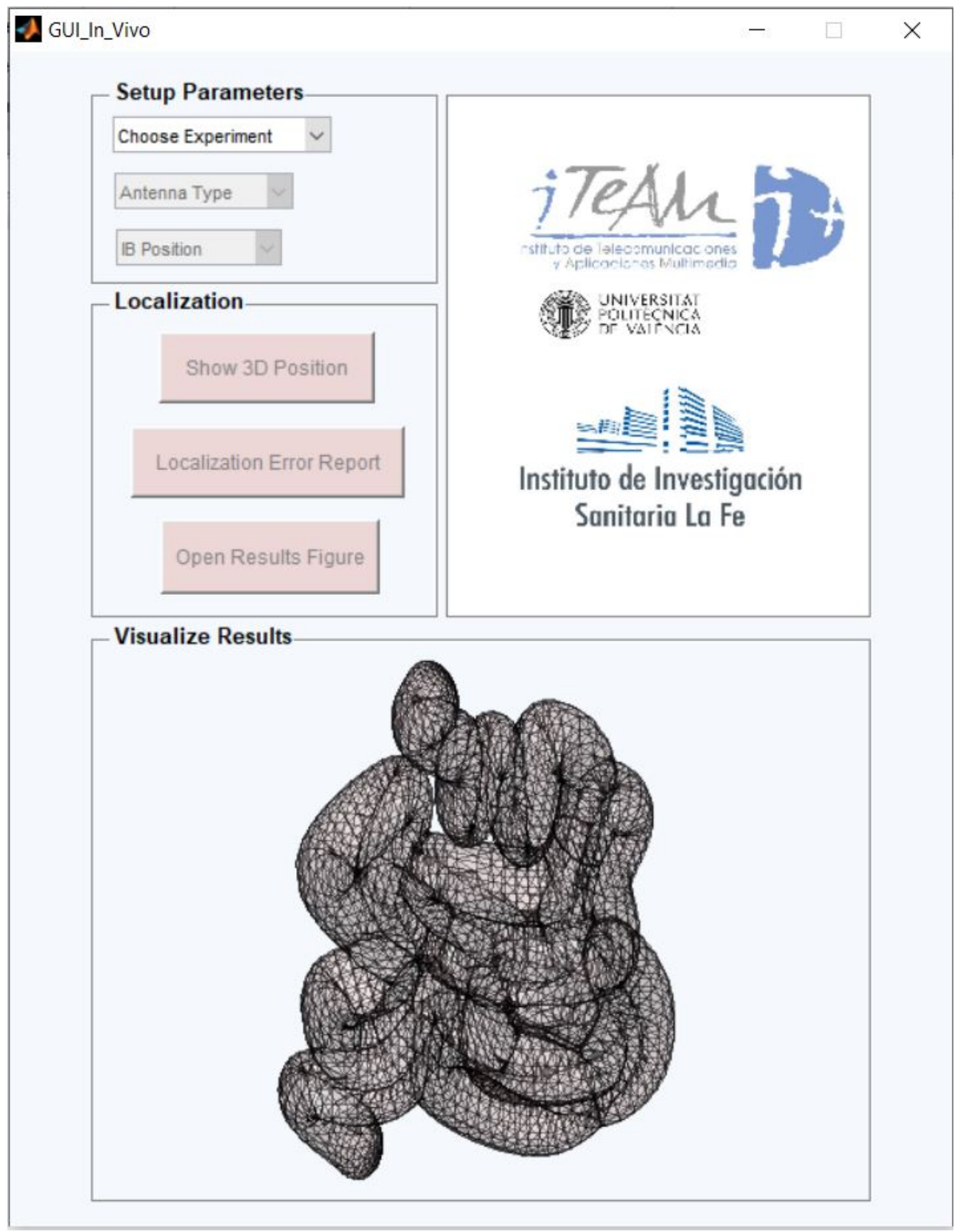

Figure A.1: Appearance of the developed Graphical User Interface (GUI) when launched 


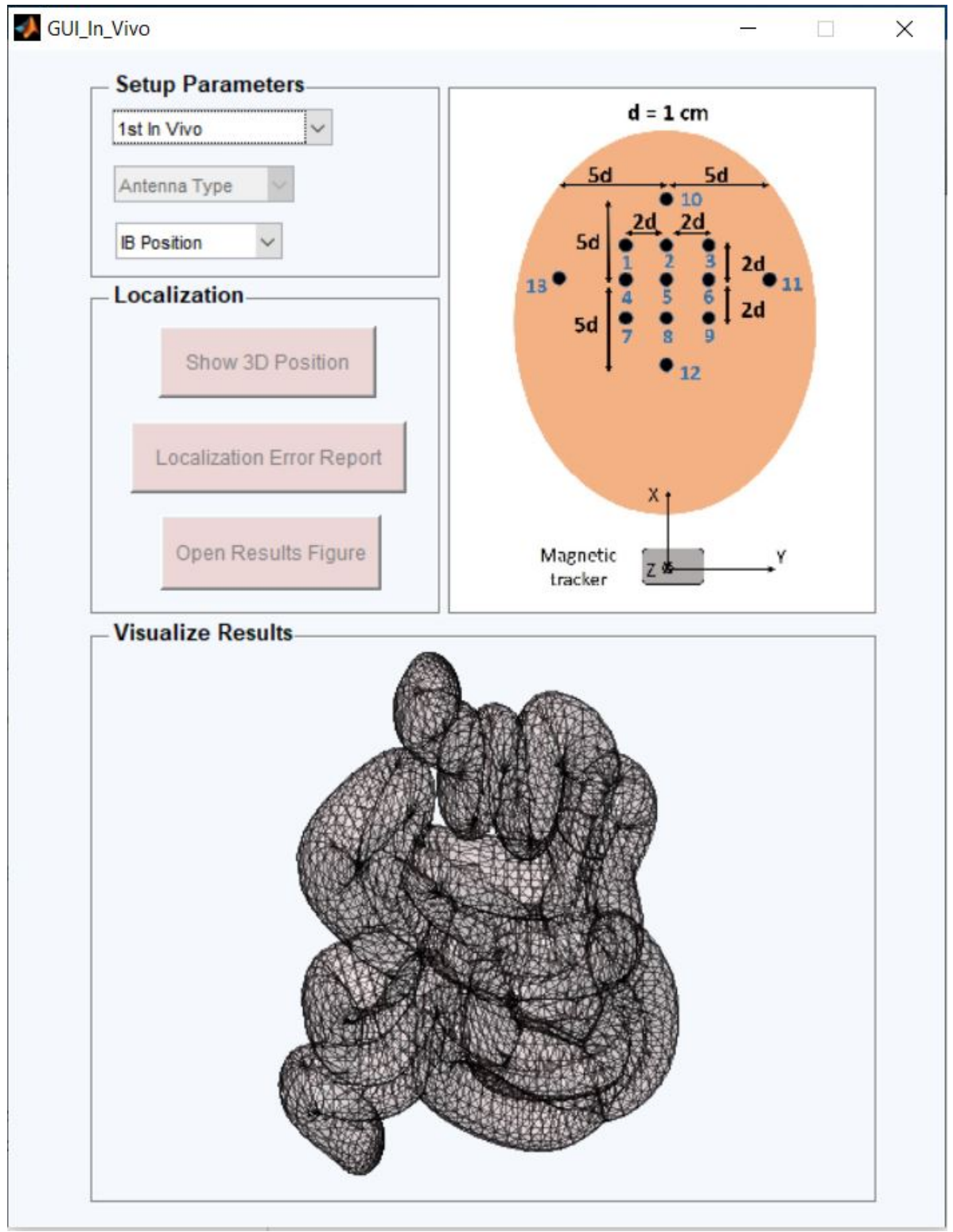

Figure A.2: GUI appearance when selecting First in vivo experiment 


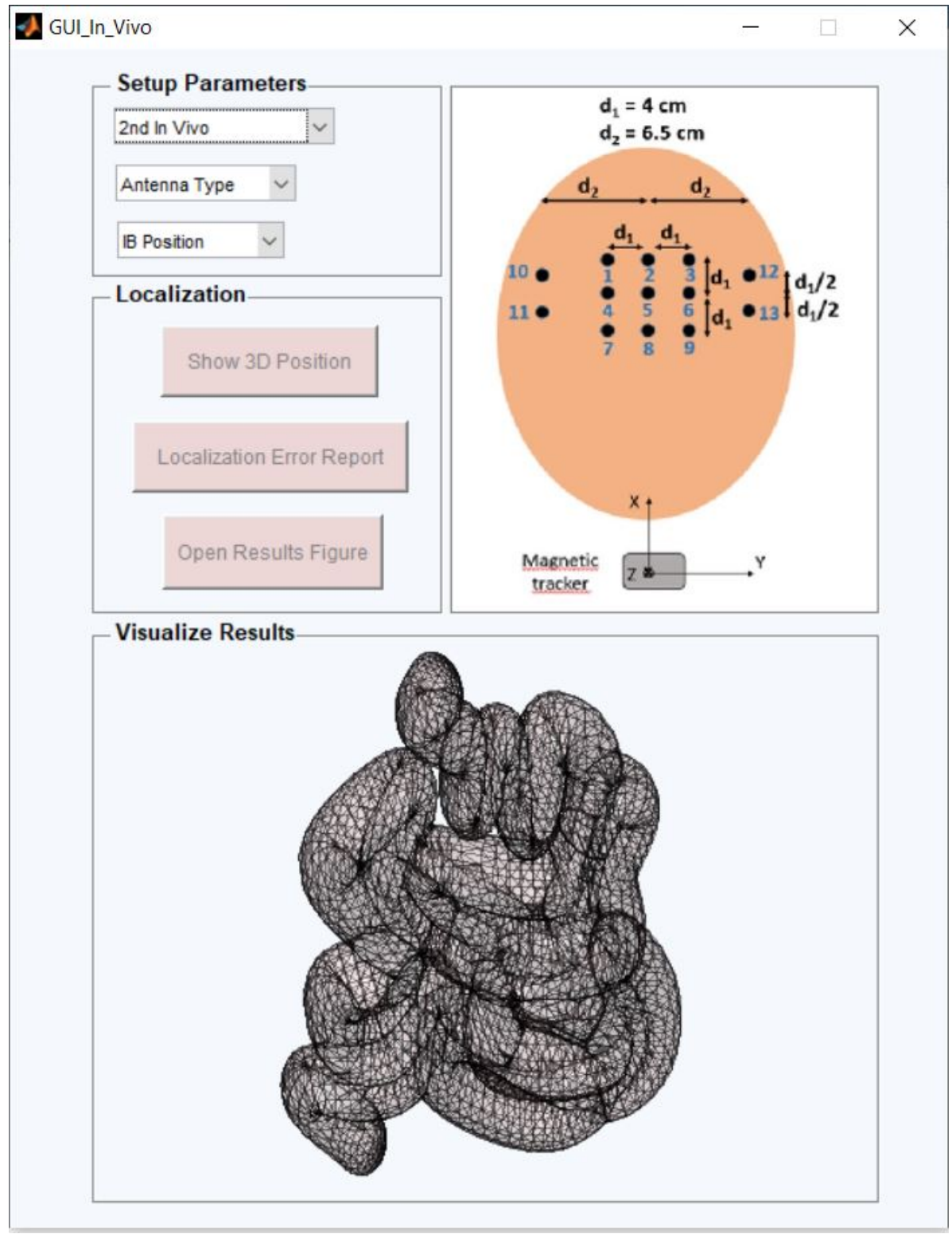

Figure A.3: GUI appearance when selecting Second in vivo experiment 


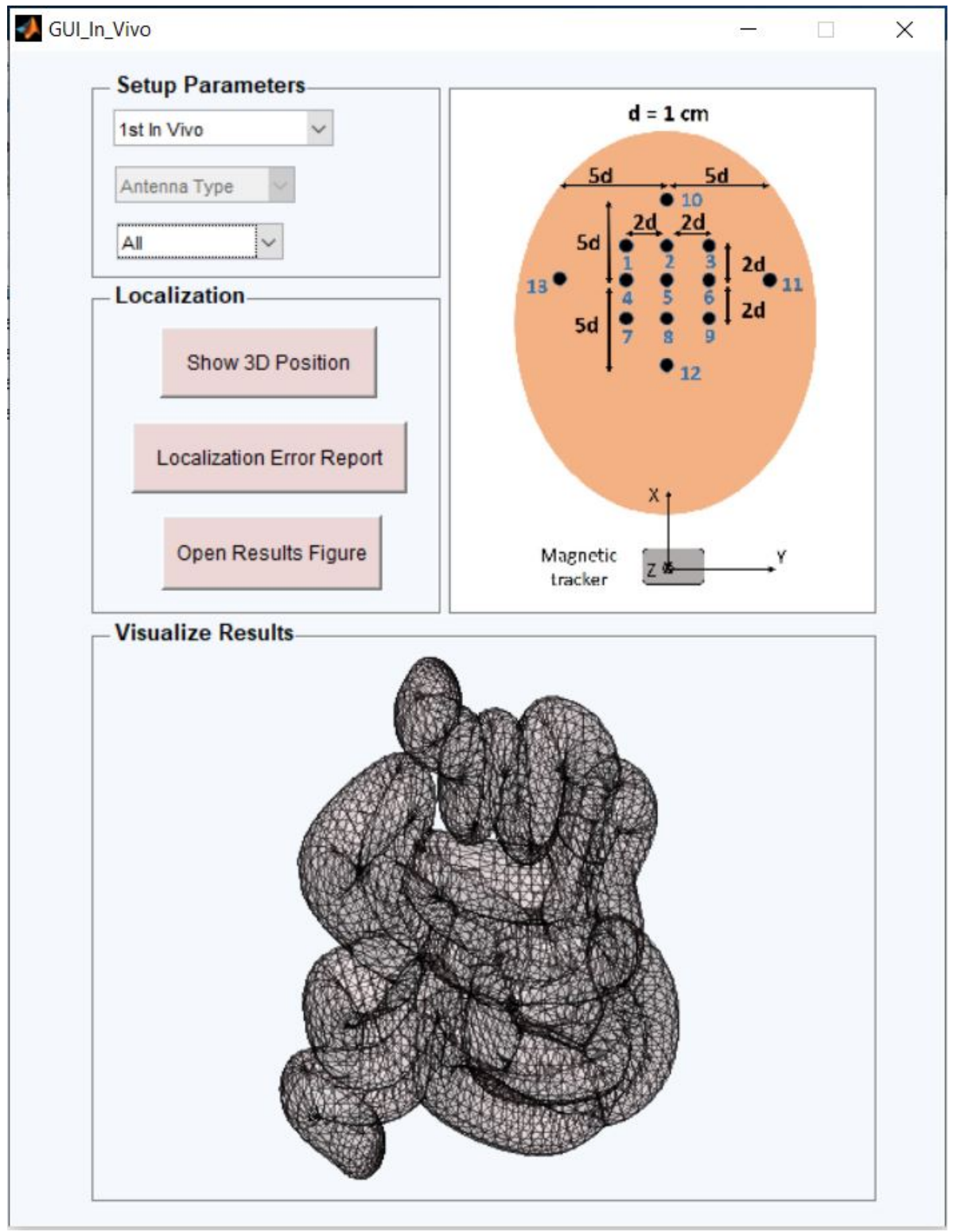

Figure A.4: GUI appearance after setting all parameters 


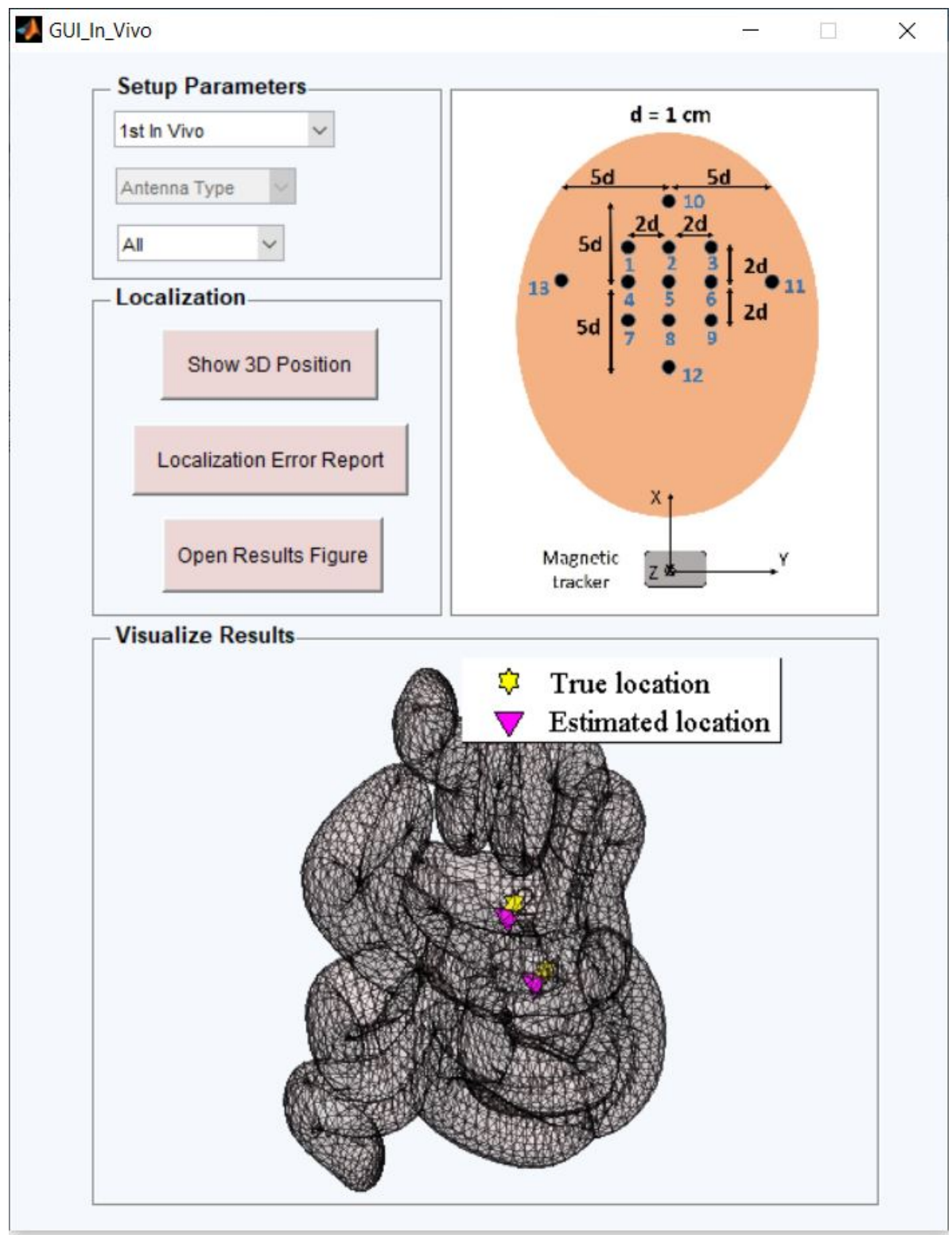

Figure A.5: GUI appearance after pushing Show 3D Position button for first in vivo experiment 


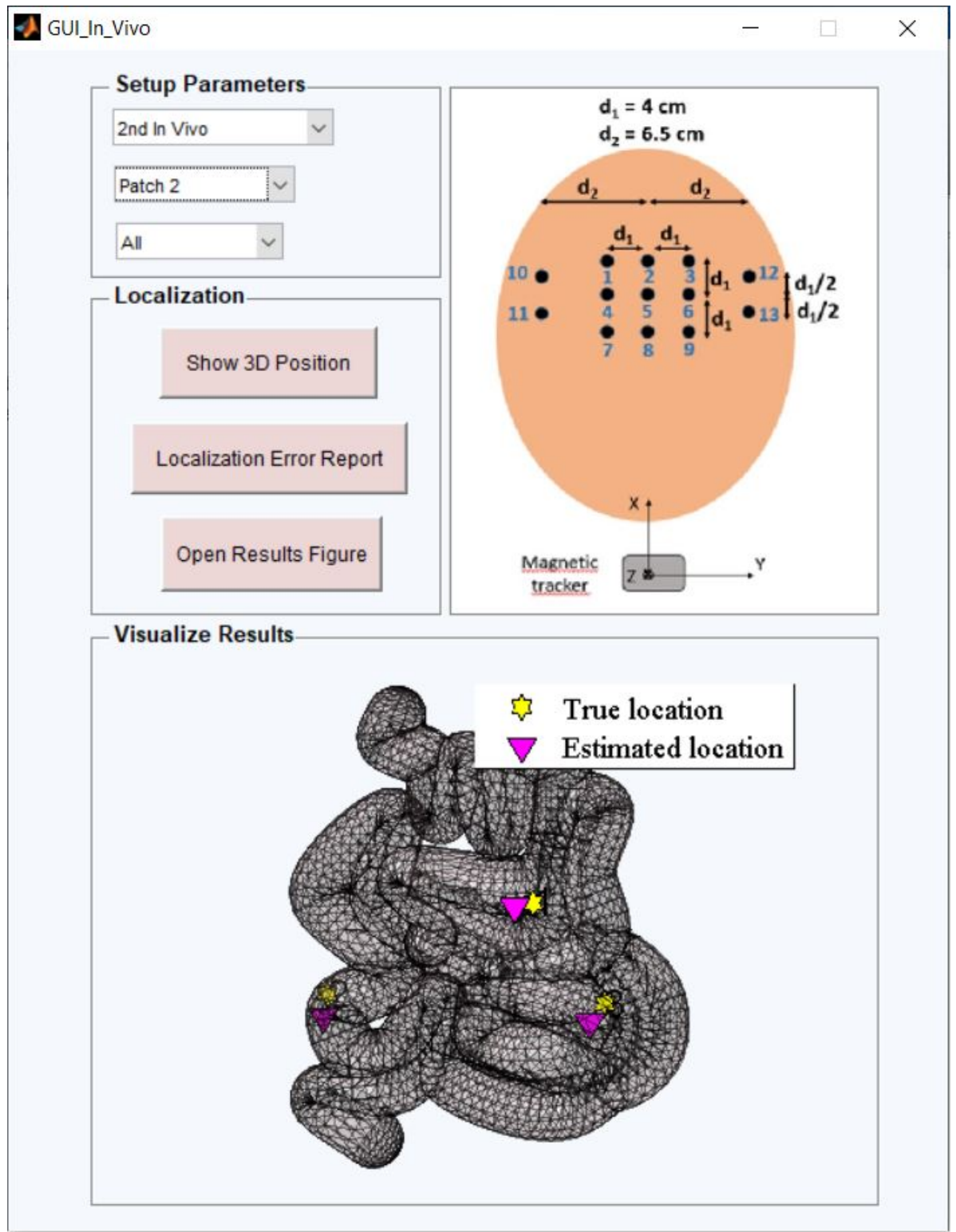

Figure A.6: GUI appearance after pushing Show 3D Position button for second in vivo experiment 


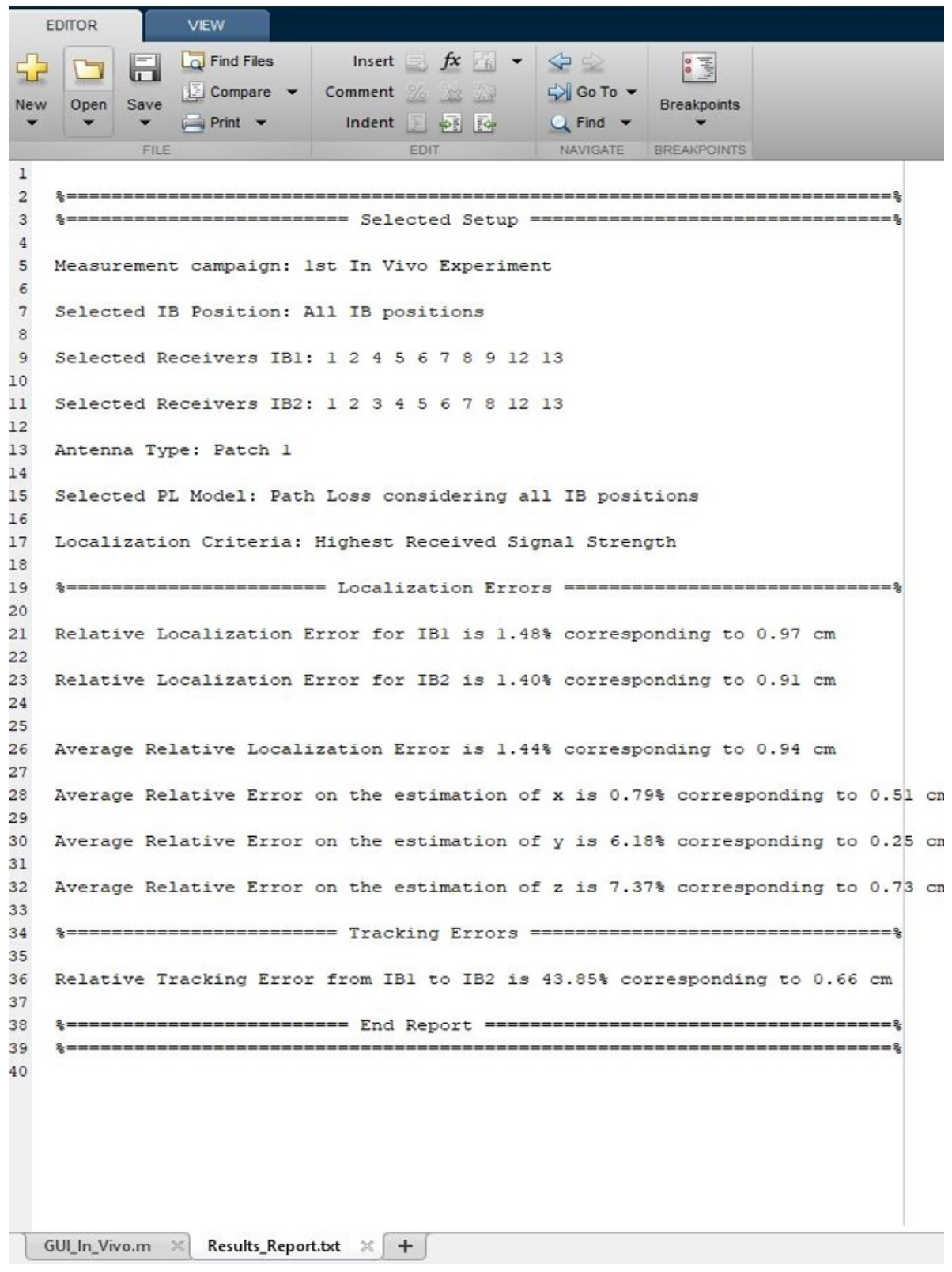

Figure A.7: Generated Localization Report after pushing Localization Error Report button 


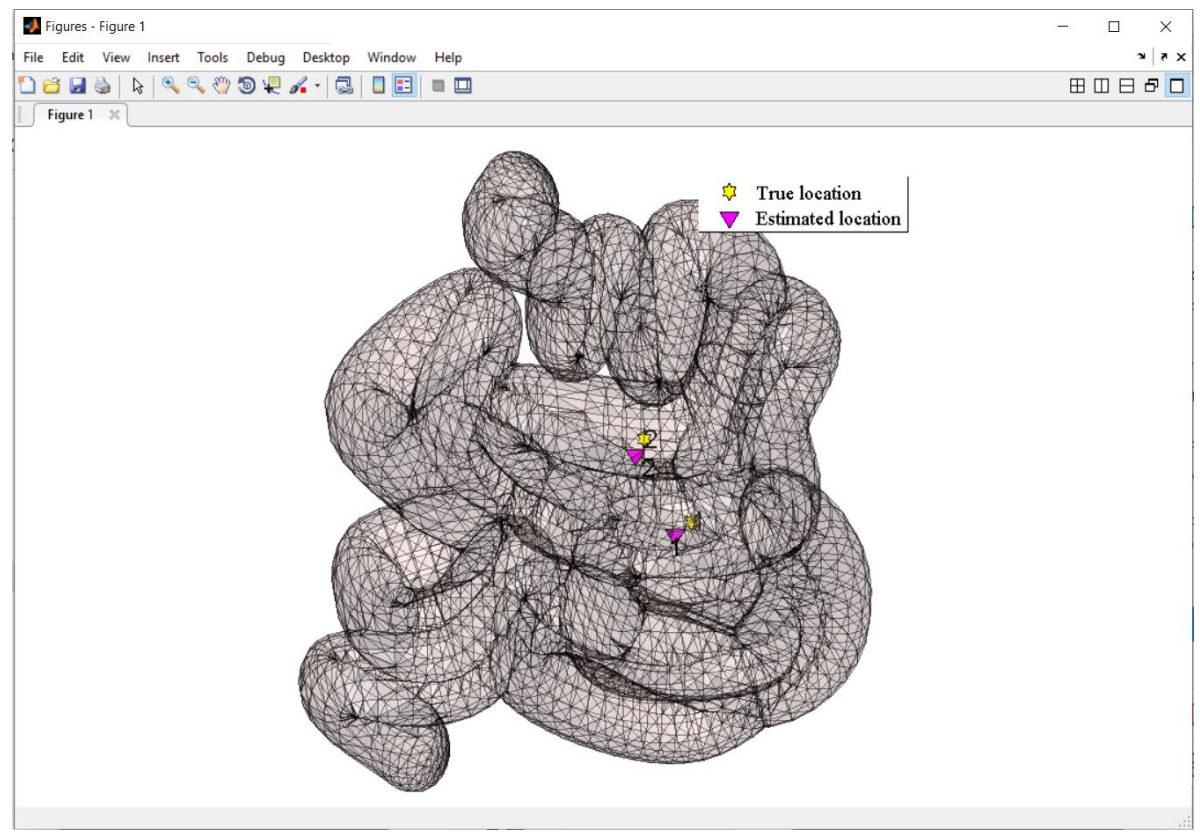

Figure A.8: Results opened in new figure window after pushing Open Results Figure button 


\section{List of Abbreviations}

AoA Angle of Arrival

BAN Body Area Network

CDF Cumulative Distribution Function

CPW Coplanar Waveguide

CST Computer Simulation Technology

DoA Direction of Arrival

FCC Federal Communications Commission

FDTD Finite Difference Time Domain

FIT Finite Integration Technique

GI Gastrointestinal

GUI Graphical User Interface

HFSS High Frequency Structure Simulator

IB2OB in-body to on-body

IFFT Inverse Fast Fourier Transform

IR Impulse Radio

IR-UWB Impulse Radio Ultra-Wideband

ISM Industrial, Scientific and Medical

ITN Innovative Training Network

LP-AMI Low Power Active Medical Implants 
LLS Linear Least Square

MICS Medical Implant Communication Service

MRI Magnetic Resonance Imaging

NLLS Non Linear Least Square

PDoA Phase Difference of Arrival

PDP Power Delay Profile

PoA Phase of Arrival

RAMCE Robot Assisted Magnetic Capsule Endoscopes

RF Radio Frequency

RMSE Root Mean Square Error

RSS Received Signal Strength

SLAM Simultaneous Localization and Mapping

SNR Signal-to-Noise Ratio

TDoA Time Difference of Arrival

ToA Time of Arrival

UGD Upper GI endoscopy

WCE Wireless Capsule Endoscopy

WiBEC Wireless In-Body Environment

WBANs Wireless Body Area Networks

WLANs Wireless Local Area Networks

UPV Universitat Politécnica de Valéncia

US United States

UWB Ultra-Wideband

VNA Vector Network Analyzer

1D One-Dimensional

2D Two-Dimensional

3D Three-Dimensional 


\section{References}

[1] Medtronic, "Medtronic products website:

https://www.medtronic.com/covidien/en-us/products/capsuleendoscopy/pillcam-sb-3-system.html."

[2] C. Garcia-Pardo, C. Andreu, A. Fornes-Leal, S. Castelló-Palacios, S. Pérez-Simbor, M. Barbi, A. Vallés-Lluch, and N. Cardona, "Ultrawideband technology for medical in-body sensor networks: An overview of the human body as a propagation medium, phantoms, and approaches for propagation analysis," IEEE Antennas and Propagation Magazine, vol. 60, no. 3, pp. 19-33, June 2018.

[3] X. Fang, M. Ramzan, Q. Wang, and D. Plettemeier, "Compact antipodal vivaldi antennas for body area communication," in Advances in Body Area Networks I, G. Fortino and Z. Wang, Eds. Cham: Springer International Publishing, 2019, pp. 357-369.

[4] S. Schostek, M. Zimmermann, J. Keller, M. Fode, M. Melbert, M. O. Schurr, T. Gottwald, and R. L. Prosst, "Telemetric real-time sensor for the detection of acute upper gastrointestinal bleeding," Biosensors and Bioelectronics, vol. 78, pp. 524-529, 2016.

[5] S. Mahmood, S. Schostek, and M. O. Schurr, "Predictive Tilt Compensation for Robot Assisted Magnetic Capsule Endoscope ," in Engineering in Medicine and Biology (EMBC), July 2019, pp. 3697-3702.

[6] V. X. Nguyen, V. T. Le Nguyen, and C. C. Nguyen, "Appropriate use of endoscopy in the diagnosis and treatment of gastrointestinal diseases: up-to-date indications for primary care providers." International journal of general medicine, vol. 3, pp. 345-57, 2010.

[7] G. Pan and L. Wang, "Swallowable wireless capsule endoscopy: Progress and technical challenges," in Gastroenterology research and practice, vol. $2012,2012$. 
[8] C. Van de Bruaene, D. De Looze, and P. Hindryckx, "Small bowel capsule endoscopy: Where are we after almost 15 years of use?" World journal of gastrointestinal endoscopy, vol. 7, no. 1, pp. 13-36, Jan. 2015.

[9] Medtronic, Medtronic RAPID Software User Manual: https://www.medtronic.com/content/dam/covidien/library/us/en/product /diagnostic-testing/rapid-v83-user-manual.pdf.

[10] "Teee standard for local and metropolitan area networks - part 15.6: Wireless body area networks," IEEE Std 802.15.6-2012, pp. 1-271, Feb. 2012.

[11] G. Ciuti, A. Menciassi, and P. Dario, "Capsule Endoscopy : From Current Achievements to Open Challenges," IEEE transactions on biomedical engineering, vol. 4, pp. 59-72, 2011.

[12] R. Li, Y. X. Guo, and G. Du, "A Conformal Circularly Polarized Antenna for Wireless Capsule Endoscope Systems," IEEE Transactions on Antennas and Propagation, vol. 66, no. 4, pp. 2119-2124, 2018.

[13] Y. Chan, M. Q.-H. Meng, X. Wang, and J. C.-Y. Wu, "A Prototype Design of a Wireless Capsule Endoscope," Applied Bionics and Biomechanics, vol. 3, no. 4, pp. 253-262, 2015.

[14] T. Report, "TR 102655 - V1.1.1 - Electromagnetic compatibility and Radio spectrum Matters (ERM); System reference document; Short Range Devices (SRD); Low Power Active Medical Implants (LP-AMI) operating in a $20 \mathrm{MHz}$ band within $2360 \mathrm{MHz}$ to $3400 \mathrm{MHz}$," vol. 1, pp. 1-55, 2008.

[15] Q. W. Jianqing Wang, Body Area Communications: Channel Modeling, Communication Systems, and EMC, 2013.

[16] M. R. Yuce, H. C. Keong, and M. S. Chae, "Wideband communication for implantable and wearable systems," IEEE Transactions on Microwave Theory and Techniques, vol. 57, no. 10, pp. 2597-2604, 2009.

[17] A. F. Molisch, "Ultra-Wide-Band Propagation Channels," Proceedings of the IEEE, vol. 97, no. 2, pp. 353-371, 2009.

[18] E. C. C. Decision, E. C. Decision, E. E. A. J. Committee, and E. C. Decision, "ECC Decision (06)04," pp. 1-15, March 2006.

[19] European Commisison (EC), "Commission Implementing Decision of 7 October 2014," Official Journal of the European Union, vol. 2014, no. 748 , pp. 48-56, 2014. 
[20] European Commisison, "Commission Decision of 21 February 2007 on allowing the use of the radio spectrum for equipment using ultra-wideband technology in a harmonised manner in the Community," Official Journal of the European Union, vol. L55, no. 1882, pp. 33-36, 2007.

[21] R. Chavez-Santiago, C. Garcia-Pardo, A. Fornes-Leal, A. Vallés-Lluch, I. Balasingham, and N. Cardona, "Ultra wideband propagation for future in-body sensor networks," in 2014 IEEE 25th Annual International Symposium on Personal, Indoor, and Mobile Radio Communication (PIMRC), Washington, DC, Sep. 2014, pp. 2160-2163.

[22] M. R. Yuce, T. Dissanayake, and H. C. Keong, "Wireless telemetry for electronic pill technology," Proceedings of IEEE Sensors, pp. 1433-1438, 2009.

[23] A. Khaleghi, R. Chávez-Santiago, and I. Balasingham, "Ultra-wideband pulse-based data communications for medical implants," IET Communications, vol. 4, no. 15, p. 1889, 2010.

[24] M. R. Yuce and T. Dissanayake, "Easy-to-Swallow Wireless Telemetry," IEEE Microwave Magazine, vol. 13, no. 6, pp. 90-101, 2012.

[25] C. Hu, M. Q. Meng, and M. Mandal, "Efficient Magnetic Localization and Orientation Technique for Capsule Endoscopy," in IEEE/RSJ International Conference on Intelligent Robots and Systems, 2005, pp. 33653370.

[26] R. Kuth, J. Reinschke, and R. Rockelein, "Method for determining the position and orientation of an endoscopy capsule guided through an examination object by using a navigating magnetic field generated by means of a navigation device," Patent US 2007/0038063 A1, Feb., 2007.

[27] G. Bao, L. Mi, and K. Pahlavan, "A Video Aided RF Localization Technique for the Wireless Capsule Endoscope (WCE) inside Small Intestine," in Proceedings of the 8th International Conference on Body Area Networks, no. February 2016, 2013, pp. 55-61.

[28] J. Lee, J. Oh, S. K. Shah, X. Yuan, and S. J. Tang, "Automatic classification of digestive organs in wireless capsule endoscopy videos," in Proceedings of the 2007 ACM symposium on Applied computing SAC 07, 2007, pp. 1041-1045.

[29] G. Bao and K. Pahlavai, "Motion estimation of the endoscopy capsule using region-based Kernel SVM classifier," in IEEE International Conference on Electro Information Technology, May 2013. 
[30] K. Pahlavan, G. Bao, Y. Ye, S. Makarov, U. Khan, P. Swar, D. Cave, A. Karellas, P. Krishnamurthy, and K. Sayrafian, "RF localization for wireless video capsule endoscopy," International Journal of Wireless Information Networks, vol. 19, no. 4, pp. 326-340, 2012.

[31] M. Pourhomayoun, M. Fowler, and Z. Jin, "A novel method for medical implant in-body localization," in Proceedings of the Annual International Conference of the IEEE Engineering in Medicine and Biology Society, EMBS, Aug.-Sep. 2012, pp. 5757-5760.

[32] U. I. Khan, K. Pahlavan, and S. Makarov, "Comparison of TOA and RSS based techniques for RF localization inside human tissue," in Proceedings of the Annual International Conference of the IEEE Engineering in Medicine and Biology Society, EMBS, Aug.-Sep. 2011, pp. 5602-5607.

[33] A. R. Nafchi, S. T. Goh, and S. A. R. Zekavat, "Circular arrays and inertial measurement unit for DOA/TOA/TDOA-based endoscopy capsule localization: Performance and complexity investigation," IEEE Sensors Journal, vol. 14, no. 11, pp. 3791-3799, 2014.

[34] Y. Yishuang Geng and K. Pahlavan, "On the accuracy of RF and image processing based hybrid localization for wireless capsule endoscopy," in 2015 IEEE Wireless Communications and Networking Conference (WCNC), Mar. 2015, pp. 452-457.

[35] R. Chandra, A. J. Johansson, and F. Tufvesson, "Localization of an RF source inside the Human body for Wireless Capsule Endoscopy," in Proceedings of the 8th International Conference on Body Area Networks, vol. 1, 2013, pp. 48-54.

[36] A. R. Nafchi, S. T. Goh, and S. A. Zekavat, "High performance DOA/TOA-based Endoscopy Capsule localization and tracking via 2D circular arrays and inertial measurement unit," IEEE International Conference on Wireless for Space and Extreme Environments, WiSEE 2013 - Conference Proceedings, pp. 1-6, Nov. 2013.

[37] N. Dey, A. S. Ashour, F. Shi, and R. S. Sherratt, "Wireless Capsule Gastrointestinal Endoscopy: Direction-of-Arrival Estimation Based Localization Survey," IEEE Reviews in Biomedical Engineering, vol. 10, pp. 2-11, 2017.

[38] M. O, T. S.H, N. Ismail, and A. Ramli, "A review on positioning techniques and technologies: A novel ai approach," Journal of Applied Sciences, vol. 9, Sep. 2009. 
[39] ITU-R, "The concept of transmission loss for radio links," Recommendation ITU-R P.341-5, vol. 1, no. Ccv, pp. 1-6, 1999.

[40] K. Sayrafian-Pour, W.-B. Yang, J. Hagedorn, J. Terrill, K. Yekeh Yazdandoost, and K. Hamaguchi, "Channel models for medical implant communication," International Journal of Wireless Information Networks, vol. 17, no. 3, pp. 105-112, Dec. 2010.

[41] A. S. Bjørnevik, "Localization and Tracking of Intestinal Paths for Wireless Capsule Endoscopy," Ph.D. dissertation, Norwegian University of Science and Technology, Trondheim, Norway, 2015.

[42] H. Farhadi, J. Atai, M. Skoglund, E. S. Nadimi, K. Pahlavan, and V. Tarokh, "An adaptive localization technique for wireless capsule endoscopy," International Symposium on Medical Information and Communication Technology, ISMICT, vol. 2016-June, pp. 1-5, 2016.

[43] Kegen Yu, "3-D Localization Error Analysis in Wireless Networks," IEEE Transactions on Wireless Communications, vol. 6, no. 10, pp. 3472-3481, 2007.

[44] W. Murphy and W. Hereman, Determination of a position in three dimensions using trilateration and approximate distances, 1995, vol. 7 .

[45] K. Arshak and F. Adepoju, "Adaptive linearized methods for tracking a moving telemetry capsule," IEEE International Symposium on Industrial Electronics, pp. 2703-2708, June 2007.

[46] W. H. Press, W. T. Vetterling, and S. A. Teukolsky, Numerical Recipes in C: The Art of Scientific Computing. Cambridge University Press, 1992.

[47] R. Fletcher and T. L. Freeman, "A modified newton method for minimization," Journal of Optimization Theory and Applications, vol. 23, no. 3, pp. 357-372, Nov. 1977.

[48] D. W. Marquardt, "An algorithm for least-squares estimation of nonlinear parameters," Journal of the Society for Industrial and Applied Mathematics, vol. 11, no. 2, pp. 431-441, June 1963.

[49] Charles L. Lawson and Richard J. Hanson, Solving Least Squares Problems, 1995.

[50] S. Tomic, M. Beko, R. Dinis, and L. Bernardo, "On target localization using combined RSS and AoA measurements," Sensors (Switzerland), vol. 18, no. 4, pp. 1-25, 2018. 
[51] J. Carlos, B. Oliveira, and A. Santos, "3-D Hybrid Localization with RSS/AoA in Wireless Sensor Networks: Centralized Approach," Tech. Rep., 2016.

[52] G. Bao, "On simultaneous localization and mapping inside the human body (body-slam)," Ph.D. dissertation, Worcester Polytechnic Institute, 2014.

[53] H. Mateen, R. Basar, A. U. Ahmed, S. Member, and M. Y. Ahmad, "Localization of Wireless Capsule Endoscope : A Systematic Review," IEEE Sensors Journal, vol. 17, no. 5, pp. 1197-1206, 2017.

[54] B. Moussakhani, J. FlaİŠm, S. Støa, I. Balasingham, and T. Ramstad, "On localisation accuracy inside the human abdomen region," IET Wireless Sensor Systems, vol. 2, no. 1, pp. 9-15, Mar. 2012.

[55] M. Kanaan and M. Suveren, "In-body ranging with ultra-wideband signals: Techniques and modeling of the ranging error," Wireless Communications and Mobile Computing, vol. 2017, 2017.

[56] Y. Ye, "Bounds on Rf Cooperative Localization for Video Capsule Endoscopy," Ph.D. dissertation, Worcester Polytechnic Institute, 2013.

[57] C. Andreu, C. Garcia-Pardo, S. Castelló-Palacios, A. Vallés-Lluch, and N. Cardona, "Frequency dependence of uwb in-body radio channel characteristics," IEEE Microwave and Wireless Components Letters, vol. 28, no. 4, pp. 359-361, Apr. 2018.

[58] G. Hartsgrove, A. Kraszewski, and A. Surowiec, "Simulated biological materials for electromagnetic radiation absorption studies," Bioelectromagnetics, vol. 8, no. 1, pp. 29-36, 1987.

[59] C. Gabriel, "Tissue equivalent material for hand phantoms," Physics in Medicine and Biology, vol. 52, no. 14, pp. 4205-4210, June 2007.

[60] S. Castelló-Palacios, C. Garcia-Pardo, A. Fornes-Leal, N. Cardona, and A. Vallés-Lluch, "Tailor-Made Tissue Phantoms Based on Acetonitrile Solutions for Microwave Applications up to $18 \mathrm{GHz}$," IEEE Transactions on Microwave Theory and Techniques, vol. 64, no. 11, pp. 3987-3994, Nov. 2016.

[61] N. Cardona, S. Castelló-Palacios, A. Fornes-Leal, C. Garcia-Pardo, and A. Vallés-Lluch, "Synthetic Model of Biological Tissues for Evaluating the Wireless Transmission of Electromagnetic Waves," Patent WO/2017/109 252, June, 2017. 
[62] S. Castelló-Palacios, C. Garcia-Pardo, A. Fornes, N. Cardona, and A. Vallés-Lluch, "Full-spectrum phantoms for $\mathrm{cm}$-wave and medical wireless communications," in 12th European Conference on Antennas and Propagation (EuCAP 2018), Apr. 2018, pp. 1-3.

[63] D. Andreuccetti, R. Fossi, and C. Petrucci, "An Internet Resource for the Calculation of the Dielectric Properties of Body Tissues in the Frequency Range 10 Hz-100 GHz, IFAC-CNR," Florence, Italy, 1997.

[64] C. Gabriel, "Compilation of the Dielectric Properties of Body Tissues at RF and Microwave Frequencies." Environmental Health, June 1996.

[65] M. Lazebnik, E. L. Madsen, G. R. Frank, and S. C. Hagness, "Tissuemimicking phantom materials for narrowband and ultrawideband microwave applications," Physics in Medicine and Biology, vol. 50, no. 18, pp. 4245-4258, Aug. 2005.

[66] H. Yamamoto, J. Zhou, and T. Kobayashi, "Ultra wideband electromagnetic phantoms for antennas and propagation studies," IEICE Transactions on Fundamentals of Electronics, Communications and Computer Sciences, vol. E91-A, no. 11, pp. 3173-3182, Nov. 2008.

[67] C. Andreu, C. Garcia-Pardo, A. Fornes-Leal, M. Cabedo-Fabrés, and N. Cardona, "UWB In-Body Channel Performance by Using a Direct Antenna Designing Procedure," in 11th European Conference on Antennas and Propagation (EUCAP), Mar. 2017, pp. 278-282.

[68] C. Tarín, P. Martí, L. Traver, N. Cardona, J. A. Díaz, and E. Antonino, "UWB channel measurements for hand-portable devices: A comparative study," in IEEE International Symposium on Personal, Indoor and Mobile Radio Communications, PIMRC, Nov. 2007.

[69] S. Pérez-Simbor, M. Barbi, M. Ramzan, X. Fang, C. Garcia-Pardo, N. Cardona, Q. Wang, N. Neumann, and D. Plettemeier, "Experimental path loss models comparison and localization of wireless endoscopic capsule in the ultra wideband frequency band," in 13th EAI International Conference on Body Area Networks (BodyNets), Oulu, Finland, Oct. 2018.

[70] S. Pérez-Simbor, M. Barbi, C. Garcia-Pardo, S. Castelló-Palacios, and N. Cardona, "Initial uwb in-body channel characterization using a novel multilayer phantom measurement setup," in 2018 IEEE Wireless Communications and Networking Conference Workshops (WCNCW), Apr. 2018, pp. 384-389.

[71] CST, AG, Germany, "CST STUDIO SUITE," www.cst.com. 
[72] P. A. Floor, R. Chavez-Santiago, S. Brovoll, O. Aardal, J. Bergsland, O. H. N. Grymyr, P. S. Halvorsen, R. Palomar, D. Plettemeier, S. Hamran, T. A. Ramstad, and I. Balasingham, "In-body to on-body ultrawideband propagation model derived from measurements in living animals," IEEE Journal of Biomedical and Health Informatics, vol. 19, no. 3, pp. 938-948, May 2015.

[73] E. Miralles Gómez, "Estudio, diseño y optimización de antena UWB para comunicaciones intracorporales," Master's thesis, UPV, Valencia, Spain, 2016.

[74] C. Andreu Estellés, "UWB Radio Channel and Diversity Characterization for Wireless Implanted Devices," Ph.D. dissertation, UPV, Valencia, Spain, 2018.

[75] H. Benchakroun, M. Cabedo-Fabrés, A. Latif, and M. Ferrando-Bataller, "Novel technique for the design of uwb monopoles with reflector for directive ban applications," International journal of Antennas and Propagation, 2018.

[76] H. Benchakroun, M. Cabedo-Fabrés, M. Ferrando-Bataller, Z. Mahlaoui, and A. Latif, "New approach for the design of uwb monopoles with directive radiation for ban applications," in 12th European Conference on Antennas and Propagation (EuCAP 2018), Apr. 2018, pp. 1-5.

[77] I. Dove, "Analysis of Radio Propagation Inside the Human Body for in-Body Localization Purposes," Master's thesis, Netherlands, pp. 1-12, 2014.

[78] M. Kawasaki and R. Kohno, "A toa based positioning technique of medical implanted devices," in 3rd International Symposium on Medical Information and Communication Technology, (ISMICT), Dec. 2009.

[79] V. Kumar and S. Kumar Dhull, "Techniques of Direction of Arrival Estimation: A Review," IUP Journal of Electrical and Electronics Engineering, vol. 9, no. 1, pp. 44-56, May 2016.

[80] A. Bjornevik, P. Anders Floor, and I. Balasingham, "On path length estimation for wireless capsule endoscopy," in 2018 12th International Symposium on Medical Information and Communication Technology (ISMICT), Mar. 2018, pp. 1-5.

[81] I. Umay, B. Fidan, and B. Barshan, "Localization and tracking of implantable biomedical sensors," Sensors (Switzerland), vol. 17, no. 3, pp. $1-20,2017$. 
[82] T. D. Than, G. Alici, H. Zhou and W. Li, "A Review of Localization Systems for Robotic Endoscopic Capsules," IEEE Transactions on Biomedical Engineering, vol. 59, no. 9, 2012.

[83] M. Barbi, S. Pérez-Simbor, C. Garcia-Pardo, C. Andreu, and N. Cardona, "Localization for capsule endoscopy at uwb frequencies using an experimental multilayer phantom," in 2018 IEEE Wireless Communications and Networking Conference Workshops (WCNCW), Apr. 2018, pp. 390-395.

[84] M. R. Basar, F. Malek, K. M. Juni, M. S. Idris, and M. I. M. Saleh, "Ingestible wireless capsule technology: A review of development and future indication," International Journal of Antennas and Propagation, vol. 2012, 2012.

[85] M. Barbi, C. Garcia-Pardo, N. Cardona, A. Nevarez, V. Pons, and M. Frasson, "Impact of receivers location on the accuracy of capsule endoscope localization," in 2018 IEEE 29th Annual International Symposium on Personal, Indoor and Mobile Radio Communications (PIMRC), Sep. 2018, pp. 340-344.

[86] M. Barbi, S. Pérez-Simbor, C. Garcia-Pardo, and N. Cardona, "Analysis of the localization error for capsule endoscopy applications at uwb frequencies," in 2019 13th International Symposium on Medical Information and Communication Technology (ISMICT), May 2019, pp. 1-6.

[87] N. C. G. C. (UK), Acute Upper Gastrointestinal Bleeding: Management. London: Royal College of Physicians (UK), 2012, vol. 141, no. June.

[88] E. F. Mostafa and A. N. Mohammad, "Incidence and predictors of rebleeding after band ligation of oesophageal varices," Arab Journal of Gastroenterology, vol. 15, no. 3, pp. 135 - 141, 2014.

[89] J. E. J. Krige, U. K. Kotze, G. Distiller, J. M. Shaw, and P. C. Bornman, "Predictive factors for rebleeding and death in alcoholic cirrhotic patients with acute variceal bleeding: A multivariate analysis," World Journal of Surgery, vol. 33, no. 10, pp. 2127-2135, Oct. 2009.

[90] K. Jung and W. Moon, "Role of endoscopy in acute gastrointestinal bleeding in real clinical practice: An evidence-based review." World journal of gastrointestinal endoscopy, vol. 11, no. 2, pp. 68-83, Feb. 2019.

[91] M. Rubin, S. A. Hussain, A. Shalomov, R. A. Cortes, M. S. Smith, and S. H. Kim, "Live view video capsule endoscopy enables risk stratification of patients with acute upper gi bleeding in the emergency room: A pilot 
study," Digestive Diseases and Sciences, vol. 56, no. 3, pp. 786-791, Mar. 2011.

[92] A. C. Meltzer, M. A. Ali, R. B. Kresiberg, G. Patel, J. P. Smith, J. M. Pines, and D. E. Fleischer, "Video Capsule Endoscopy in the Emergency Department: A Prospective Study of Acute Upper Gastrointestinal Hemorrhage," Annals of Emergency Medicine, vol. 61, no. 4, pp. 438-443.e1, Apr. 2013.

[93] J. J. Sung, P. W. Chiu, F. K. L. Chan, J. Y. Lau, K.-l. Goh, L. H. Ho, H.-y. Jung, J. D. Sollano, T. Gotoda, N. Reddy, R. Singh, K. Sugano, K.-c. Wu, C.-Y. Wu, D. J. Bjorkman, D. M. Jensen, E. J. Kuipers, and A. Lanas, "Asia-Pacific working group consensus on non-variceal upper gastrointestinal bleeding: an update 2018," Gut, vol. 67, no. 10, pp. 1757-1768, Oct. 2018.

[94] S. Schostek, M. Zimmermann, J. Keller, M. Fode, M. Melbert, M. O. Schurr, T. Gottwald, and R. L. Prosst, "Volunteer Case Series of a New Telemetric Sensor for Blood Detection in the Upper Gastrointestinal Tract: The HemoPill," Digestive Diseases and Sciences, vol. 61, no. 10, pp. 2956-2962, 2016.

[95] S. Schostek, M. Zimmermann, J. Keller, M. Fode, M. Melbert, R. L. Prosst, T. Gottwald, and M. O. Schurr, "Pre-clinical study on a telemetric gastric sensor for recognition of acute upper gastrointestinal bleeding : the " HemoPill monitor "," Surgical Endoscopy, May. 2019.

[96] "Cancer Screening Programmes: https://www.rdehospital.nhs.uk/docs /patients/bowel_cancer_screening/Colonoscopy.pdf," Tech. Rep.

[97] L. Wang, C. Hu, M. Ieee, L. Tian, M. Li, and Q. Max, "A Novel Radio Propagation Radiation Model for Location of the Capsule in GI Tract," 2009 IEEE International Conference on Robotics and Biomimetics (ROBIO), pp. 2332-2337, Dec. 2009.

[98] A. Johansson, "Wireless communication with medical implants: Antennas and propagation," Ph.D. dissertation, Department of Electrical and Information Technology, Lund, Sweden, 2004.

[99] K. Sayrafian, "Project: IEEE P802.15 Working Group for Wireless Personal Area Networks (WPANs) Submission Title: [A Statistical Path Loss Model for MICS]," Tech. Rep., Sep. 2008.

[100] K. Krhac, K. Sayrafian, M. Alasti, K. Y. Yazdandoost, and D. Simunic, "A study of capsule endoscopy orientation estimation using received signal strength," in 2018 IEEE 29th Annual International Symposium 
on Personal, Indoor and Mobile Radio Communications (PIMRC), Sep. 2018, pp. 345-349.

[101] A. A. Balabel, H. A. Malhat, and S. H. Zainud-Deen, "Circularlypolarized 4-arm curl antenna for wireless capsule endoscopy," in 2018 35th National Radio Science Conference (NRSC), Mar. 2018, pp. 22-29. 